\title{
Towards de-escalation of axillary management after neoadjuvant chemotherapy in breast cancer
}

Citation for published version (APA):

Simons, J. M. (2019). Towards de-escalation of axillary management after neoadjuvant chemotherapy in breast cancer. [Doctoral Thesis, Maastricht University]. ProefschriftMaken. https://doi.org/10.26481/dis.20191218js

Document status and date:

Published: 01/01/2019

DOI:

10.26481/dis.20191218js

Document Version:

Publisher's PDF, also known as Version of record

\section{Please check the document version of this publication:}

- A submitted manuscript is the version of the article upon submission and before peer-review. There can be important differences between the submitted version and the official published version of record.

People interested in the research are advised to contact the author for the final version of the publication, or visit the DOI to the publisher's website.

- The final author version and the galley proof are versions of the publication after peer review.

- The final published version features the final layout of the paper including the volume, issue and page numbers.

Link to publication

\footnotetext{
General rights rights.

- You may freely distribute the URL identifying the publication in the public portal. please follow below link for the End User Agreement:

www.umlib.nl/taverne-license

Take down policy

If you believe that this document breaches copyright please contact us at:

repository@maastrichtuniversity.nl

providing details and we will investigate your claim.
}

Copyright and moral rights for the publications made accessible in the public portal are retained by the authors and/or other copyright owners and it is a condition of accessing publications that users recognise and abide by the legal requirements associated with these

- Users may download and print one copy of any publication from the public portal for the purpose of private study or research.

- You may not further distribute the material or use it for any profit-making activity or commercial gain

If the publication is distributed under the terms of Article $25 \mathrm{fa}$ of the Dutch Copyright Act, indicated by the "Taverne" license above, 
Towards de-escalation of axillary management after neoadjuvant chemotherapy in breast cancer 


\section{Colofon}

(C) Copyright Janine Simons, Maastricht, 2019.

All rights reserved. No part of this book may be reproduced or transmitted in any form or by any means, without prior permission in writing by the author, or when appropriate, by the publishers of the publications.

ISBN 978-94-6380-651-0

Cover/design: Jannet Verhoeff \& Janine Simons

Layout: Tiny Wouters

Production: Proefschriftmaken.nl

Parts of the research described in this thesis were financially supported by a grant from Alpe d'Huzes / KWF Kankerbestrijding (AZB 2015-8023).

Financial support for publication of this thesis was kindly provided by Agendia, ChipSoft, Pfizer and Maastricht University. 


\title{
Towards de-escalation of axillary management after neoadjuvant chemotherapy in breast cancer
}

\author{
PROEFSCHRIFT \\ ter verkrijging van de graad van doctor aan de Universiteit Maastricht, \\ op gezag van de Rector Magnificus, Prof. Dr. Rianne M. Letschert, \\ volgens het besluit van het College van Decanen, \\ in het openbaar te verdedigen \\ op woensdag 18 december 2019 om 14.00 uur
}

door

Janine Marilisa Simons 


\section{Promotor}

Dr. M.L. Smidt (ius promovendi)

\section{Copromotores}

Dr. E.J.T. Luiten (Amphia Ziekenhuis)

Dr. L.B. Koppert (Erasmus Medisch Centrum)

\section{Beoordelingscommissie}

Prof. dr. L.P.S. Stassen (voorzitter)

Dr. H.J.G.D. van den Bongard (Amsterdam Universitair Medische Centra)

Prof. dr. R.M. Pijnappel (Universitair Medisch Centrum Utrecht)

Prof. dr. V.C.G. Tjan-Heijnen 



\section{Contents}

Chapter 1 Introduction and thesis outline 9

Partly published in NTVO 2017;14:53-4.

Part I De-escalation of axillary management after neoadjuvant systemic therapy

Chapter 2 De-escalation of axillary surgery in breast cancer patients treated in the neoadjuvant setting: a Dutch population-based study Submitted.

Chapter 3 Patterns of axillary staging and management in clinically node positive breast cancer patients treated with neoadjuvant systemic therapy: results of a survey amongst breast cancer specialists

Eur J Surg Oncol. 2019 Aug. Doi: 10.1016/j.ejso.2019.08.012.

Part II In search of an accurate less invasive axillary staging method for node positive breast cancer

Chapter 4 Diagnostic accuracy of different surgical procedures for axillary staging after neoadjuvant systemic therapy in node-positive breast cancer: a systematic review and meta-analysis Ann Surg. 2019 Mar;269(3):432-442.

Chapter 5 A novel less-invasive approach for axillary staging after 79 neoadjuvant chemotherapy in patients with axillary node-positive breast cancer by combining radioactive iodine seed localization in the axilla with the sentinel node procedure (RISAS): a Dutch prospective multicenter validation study Clin Breast Cancer. 2017 Aug;17(5):399-402.

Chapter 6 Excision of both the pretreatment marked positive node and sentinel nodes improves axillary staging after neoadjuvant systemic therapy Br J Surg. 2019 Oct. Doi: 10.1002/bjs.11320. 
Chapter 7 Correlation between pathologic complete response in the breast and absence of axillary lymph node metastases after neoadjuvant systemic therapy

Ann Surg. 2018 Dec. Doi: 10.1097/SLA.0000000000003126.

Chapter 8 Prognosis of residual axillary disease after neoadjuvant chemotherapy in clinically node-positive breast cancer patients: isolated tumor cells and micrometastases carry a better prognosis than macrometastases Breast Cancer Res Treat. 2017;163(1):159-166.

Chapter 9 Discussion and future perspectives

Chapter 10 Dutch summary | Nederlandse samenvatting

Editorial: "Okselbehandeling van primair klierpositief mammacarcinoom na neoadjuvante systemische therapie: is minder wel veilig?" (NTvO 2017;14:53-4)

List of publications

Acknowledgements | Dankwoord 



\section{CHAPTER}

Introduction and thesis outline 


\section{INTRODUCTION}

This thesis describes practices regarding axillary management in patients with breast cancer treated with neoadjuvant chemotherapy. It focuses on identifying the most optimal surgical axillary staging procedure, i.e. one that improves quality of life by being less invasive while at the same time guarantees oncologic safety by being highly accurate. The ultimate aim is to help shape response-based axillary management. This introduction provides background information regarding this topic, followed by a brief outline of this thesis.

\section{Trend of de-escalation of surgery in breast cancer}

Breast cancer is the most frequently diagnosed malignancy amongst women worldwide. ${ }^{1}$ Over the past decades, survival rates of patients with breast cancer have increased considerably following improvements in screening, diagnostics and treatment. In addition, quality of life has improved as a result of a decrease in surgical morbidity with the development of less invasive surgical options. With the increasing number of breast cancer survivors, quality of life post-treatment becomes more and more important.

Radical mastectomy used to be standard of care in breast cancer treatment. This procedure, introduced in 1894 by Halsted, includes removal of the whole breast, the pectoral muscles and all axillary lymph nodes. Over time, the procedure was modified to the current standard of mastectomy in which the pectoral muscles are spared. In the '70s, a major step in de-escalating breast cancer surgery was achieved with the introduction of breast conserving surgery. This technique proved to be non-inferior to mastectomy (even after long-term follow up of over 25 years), provided that breast conserving surgery is followed by radiotherapy. ${ }^{2,3}$ More recent research even suggests that breast conserving surgery may be associated with improved breast cancer specific and overall survival. ${ }^{4}$ In the early 90s, the sentinel lymph node dissection (SLND) was introduced as a less invasive procedure to stage the axilla in clinically node negative (cNO) patients. ${ }^{5}$ Patients without axillary metastases in the SLNs, could thus be spared axillary lymph node dissection (ALND) which causes significant upper extremity morbidity. ${ }^{6-8}$ Following results from ACOSOG Z011, IBCSG 23-01 and AMAROS, indications for omitting ALND expanded, as SLND only was reported to be non-inferior to completion ALND in case of $\leq 2$ positive SLNs. ${ }^{9-11}$ Importantly, all patients received adjuvant chemotherapy and mastectomy patients were not included or comprised only small numbers of the study population. For cNO patients with 3 or more positive SLNs or primary clinically node positive $(\mathrm{cN}+)$ patients, ALND remained standard of care. 


\section{Timing of chemotherapy}

In breast cancer, chemotherapy was traditionally administered following surgery. In the 70s, chemotherapy given prior to surgery, i.e. neoadjuvant chemotherapy, was introduced. Advantages, while achieving similar survival rates, are in vivo tumor response and the possibility to downsize tumor volume disease. ${ }^{12-14}$ Nowadays, neoadjuvant chemotherapy is not only used to convert irresectable breast cancer to operable breast cancer, but also to downsize locally advanced operable breast cancer as well as early stage breast cancer. ${ }^{15}$ Neoadjuvant chemotherapy can even result in a pathologic complete response ( $\mathrm{pCR}$ ), which means that there are no vital tumor cells left. This creates potential to further de-escalate surgical treatment.

\section{De-escalation of surgery after neoadjuvant chemotherapy}

Neoadjuvant chemotherapy has enabled increased use of breast conserving surgery. ${ }^{12}$ Additionally, when performed after chemotherapy compared to the primary setting, breast conserving surgery may involve smaller amounts of tissue. This contributes to improved cosmetic outcome. Currently, trials are even investigating the accuracy of non-operative breast management, i.e. identifying breast $\mathrm{PCR}$ with biopsy, to make optimal use of treatment response. ${ }^{16-19}$

Regarding the treatment of the axilla, de-escalation of surgery guided by treatment response is still an area of controversy. In cNO patients treated with neodjuvant chemotherapy, SLND is increasingly performed after chemotherapy. Since the vast majority of cNO patients are SLND-negative after chemotherapy, it is questioned whether axillary surgery can be completely omitted in subgroups among these patients. ${ }^{20,21}$ Regarding cNO patients who are SLND-positive after chemotherapy, it is yet unknown whether SLND only is non-inferior to completion ALND. This is due to the fact that trials like Z0011 did not include patients treated with neoadjuvant chemotherapy. A positive SLND after chemotherapy probably indicates resistance to the administered therapy and may even indicate the need for further (systemic) adjuvant treatment.

In $\mathrm{cN}+$ patients treated with neoadjuvant chemotherapy, de-escalation of axillary surgery is also topic of debate. Approximately $36 \%$ of $\mathrm{cN}+$ patients treated with neoadjuvant chemotherapy achieve an axillary $\mathrm{pCR} .^{22}$ These patients will likely not benefit from ALND. Noninvasive techniques, including ultrasound, MRI or PET-CT, are currently not able to accurately identify axillary $\mathrm{PCR}$. In case of an axillary complete response on imaging, residual disease may actually still be present in the axilla. Hence, several less-invasive staging procedures have been proposed as alternatives to ALND. SLND has been thoroughly studied for its diagnostic accuracy in identifying axillary $\mathrm{pCR}$ in $\mathrm{cN}+$ patients and is associated with high false negative rates (FNR). This indicates that residual disease can be missed when the SLN is negative. ${ }^{22-25}$ The high 
FNR may be caused by 'false' SLNs, caused by metastases blocking normal lymph drainage. In 2010, the MARI procedure (Marking Axillary lymph nodes with a Radioactive lodine seed) was introduced ${ }^{26}$, in which the pathologically proven positive lymph node is marked with a radioactive iodine seed prior to chemotherapy and selectively removed after chemotherapy. In a single-center validation trial of $100 \mathrm{cN}+$ patients, an FNR of $7 \%$ and a negative predictive value (NPV) of $83 \%$ was reported. The MARI procedure is not hampered by 'false' SLNs, however, residual axillary (therapy-resistant) disease can still be missed with the MARI procedure. ${ }^{27}$ Based on the NPV of $83 \%, 1$ out of 6 patients with a negative MARI node will in fact have residual axillary disease in the remaining nodes. A protocol has been published which suggests combining the outcome of MARI with the number of positive lymph nodes on PET-CT (performed prior to chemotherapy) to guide the extent of further axillary management after neoadjuvant chemotherapy. ${ }^{28}$ Such a protocol results in a decrease in ALND $^{29}$, but it is yet unknown whether omission of ALND after chemotherapy in $\mathrm{CN}+$ patients will impact long-term prognosis. In 2016, results of the Targeted Axillary Dissection (TAD) were published ${ }^{30}$, in which both the pathologically proven positive lymph node (marked prior to chemotherapy) and the SLNs are removed. The marked node was not an SLN in $23 \%$ of patients and an excellent FNR (2\%) and NPV (97\%) were reported for TAD. However, this single-center study consisted of a small cohort and patients were retrospectively identified from a prospective registry. ${ }^{30}$ The Dutch multicenter RISAS trial was set up in 2015 (funded by KWF Kankerbestrijding) to validate the combination of MARI and SLND in $225 \mathrm{cN}+$ patients. The RISAS study protocol is presented in this thesis. Whilst awaiting results of ongoing trials, several guidelines have already accepted different less invasive staging procedures as an alternative to ALND.

In conclusion, the optimal axillary management after neoadjuvant chemotherapy is yet unknown for both $\mathrm{cNO}$ and $\mathrm{cN}+$ patients. There is a need for response-based management of the axilla. This thesis aims to provide further insight in current practices regarding axillary management, different less invasive axillary staging procedures, prediction of axillary PCR based on treatment-response in the breast, and the relation between the extent of residual axillary disease and long-term prognosis. Thereby, adding to further delineating response-based axillary management.

\section{Thesis outline}

In the first part of this thesis, an overview is provided of trends in and opinions on omission of ALND after neoadjuvant chemotherapy. In chapter 2, a population-based study is presented in which trends in de-escalation of axillary surgery after neoadjuvant chemotherapy are assessed. This study focuses on changes over time in rates of ALND. In chapter 3, current practices regarding axillary staging and 
management in $\mathrm{cN}+$ patients treated with neoadjuvant chemotherapy were assessed by an online survey amongst breast cancer specialists.

The second part of this thesis focuses on $\mathrm{cN}+$ patients. In chapter 4 , an overview of the diagnostic accuracy of currently available less invasive axillary staging procedures is provided in a systematic review and meta-analysis. In chapter 5 , the study protocol of RISAS is presented, a Dutch multicenter prospective validation trial in which the accuracy of MARI in combination with SLND is assessed. In chapter 6, a retrospective multicenter evaluation of axillary staging based on MARI in combination with SLND is reported.

The third part of this thesis focuses on prediction of axillary $\mathrm{PCR}$ and on prognostic implications of residual axillary disease. Chapter 7 reports on the association between breast $\mathrm{pCR}$ and axillary $\mathrm{pCR}$ in $\mathrm{cNO}$ and $\mathrm{cN}+$ patients treated with neoadjuvant chemotherapy. In chapter 8 , prognosis of $\mathrm{cN}+$ patients treated with neoadjuvant chemotherapy is studied in relation to the extent of residual axillary disease. 


\section{REFERENCES}

1. Ferlay J, Colombet M, Soerjomataram I, et al. Estimating the global cancer incidence and mortality in 2018: GLOBOCAN sources and methods. Int J Cancer. 2019;144(8):1941-1953.

2. Fisher B, Anderson S, Bryant J, et al. Twenty-year follow-up of a randomized trial comparing total mastectomy, lumpectomy, and lumpectomy plus irradiation for the treatment of invasive breast cancer. N Engl J Med. 2002;347(16):1233-1241.

3. Veronesi U, Cascinelli N, Mariani L, et al. Twenty-year follow-up of a randomized study comparing breast-conserving surgery with radical mastectomy for early breast cancer. $N$ Engl J Med. 2002;347(16):1227-1232.

4. Lagendijk M, van Maaren MC, Saadatmand S, et al. Breast conserving therapy and mastectomy revisited: Breast cancer-specific survival and the influence of prognostic factors in 129,692 patients. Int J Cancer. 2018;142(1):165-175.

5. Krag DN, Weaver DL, Alex JC, Fairbank JT. Surgical resection and radiolocalization of the sentinel lymph node in breast cancer using a gamma probe. Surg Oncol. 1993;2(6):335-339; discussion 340.

6. Ashikaga T, Krag DN, Land SR, et al. Morbidity results from the NSABP B-32 trial comparing sentinel lymph node dissection versus axillary dissection. J Surg Oncol. 2010;102(2):111-118.

7. Krag $D$, Weaver $D$, Ashikaga $T$, et al. The sentinel node in breast cancer--a multicenter validation study. N Engl J Med. 1998;339(14):941-946.

8. Krag DN, Anderson SJ, Julian TB, et al. Sentinel-lymph-node resection compared with conventional axillary-lymph-node dissection in clinically node-negative patients with breast cancer: overall survival findings from the NSABP B-32 randomised phase 3 trial. Lancet Oncol. 2010;11(10):927-933.

9. Donker $M$, van Tienhoven $G$, Straver $M E$, et al. Radiotherapy or surgery of the axilla after a positive sentinel node in breast cancer (EORTC 10981-22023 AMAROS): a randomised, multicentre, openlabel, phase 3 non-inferiority trial. Lancet Oncol. 2014;15(12):1303-1310.

10. Galimberti V, Cole BF, Viale G, et al. Axillary dissection versus no axillary dissection in patients with breast cancer and sentinel-node micrometastases (IBCSG 23-01): 10-year follow-up of a randomised, controlled phase 3 trial. Lancet Oncol. 2018;19(10):1385-1393.

11. Giuliano AE, Ballman KV, McCall L, et al. Effect of Axillary Dissection vs No Axillary Dissection on 10Year Overall Survival Among Women With Invasive Breast Cancer and Sentinel Node Metastasis: The ACOSOG Z0011 (Alliance) Randomized Clinical Trial. JAMA. 2017;318(10):918-926.

12. Early Breast Cancer Trialists' Collaborative G. Long-term outcomes for neoadjuvant versus adjuvant chemotherapy in early breast cancer: meta-analysis of individual patient data from ten randomised trials. Lancet Oncol. 2018;19(1):27-39.

13. Cortazar $\mathrm{P}$, Zhang $\mathrm{L}$, Untch $\mathrm{M}$, et al. Pathological complete response and long-term clinical benefit in breast cancer: the CTNeoBC pooled analysis. Lancet. 2014;384(9938):164-172.

14. von Minckwitz G, Blohmer JU, Costa SD, et al. Response-guided neoadjuvant chemotherapy for breast cancer. J Clin Oncol. 2013;31(29):3623-3630.

15. Vugts G, Maaskant-Braat AJ, Nieuwenhuijzen GA, Roumen RM, Luiten EJ, Voogd AC. Patterns of Care in the Administration of Neo-adjuvant Chemotherapy for Breast Cancer. A Population-Based Study. Breast J. 2016;22(3):316-321.

16. Kuerer HM, Rauch GM, Krishnamurthy S, et al. A Clinical Feasibility Trial for Identification of Exceptional Responders in Whom Breast Cancer Surgery Can Be Eliminated Following Neoadjuvant Systemic Therapy. Ann Surg. 2018;267(5):946-951.

17. Kuerer HM, Vrancken Peeters M, Rea DW, Basik M, De Los Santos J, Heil J. Nonoperative Management for Invasive Breast Cancer After Neoadjuvant Systemic Therapy: Conceptual Basis and Fundamental International Feasibility Clinical Trials. Ann Surg Oncol. 2017;24(10):2855-2862.

18. Heil J, Sinn P, Richter H, et al. RESPONDER - diagnosis of pathological complete response by vacuumassisted biopsy after neoadjuvant chemotherapy in breast Cancer - a multicenter, confirmative, onearmed, intra-individually-controlled, open, diagnostic trial. BMC Cancer. 2018;18(1):851. 
19. van der Noordaa MEM, van Duijnhoven FH, Loo CE, et al. Identifying pathologic complete response of the breast after neoadjuvant systemic therapy with ultrasound guided biopsy to eventually omit surgery: Study design and feasibility of the MICRA trial (Minimally Invasive Complete Response Assessment). Breast. 2018;40:76-81.

20. Tadros AB, Yang WT, Krishnamurthy S, et al. Identification of Patients With Documented Pathologic Complete Response in the Breast After Neoadjuvant Chemotherapy for Omission of Axillary Surgery. JAMA Surg. 2017;152(7):665-670.

21. Barron AU, Hoskin TL, Day CN, Hwang ES, Kuerer HM, Boughey JC. Association of Low Nodal Positivity Rate Among Patients With ERBB2-Positive or Triple-Negative Breast Cancer and Breast Pathologic Complete Response to Neoadjuvant Chemotherapy. JAMA Surg. 2018;153(12):1120-1126.

22. van Nijnatten TJ, Schipper RJ, Lobbes MB, Nelemans PJ, Beets-Tan RG, Smidt ML. The diagnostic performance of sentinel lymph node biopsy in pathologically confirmed node positive breast cancer patients after neoadjuvant systemic therapy: A systematic review and meta-analysis. Eur J Surg Oncol. 2015;41(10):1278-1287.

23. Boughey JC, Suman VJ, Mittendorf EA, et al. Sentinel lymph node surgery after neoadjuvant chemotherapy in patients with node-positive breast cancer: the ACOSOG Z1071 (Alliance) clinical trial. JAMA. 2013;310(14):1455-1461.

24. Boileau JF, Poirier B, Basik M, et al. Sentinel node biopsy after neoadjuvant chemotherapy in biopsyproven node-positive breast cancer: the SN FNAC study. J Clin Oncol. 2015;33(3):258-264.

25. Kuehn T, Bauerfeind I, Fehm T, et al. Sentinel-lymph-node biopsy in patients with breast cancer before and after neoadjuvant chemotherapy (SENTINA): a prospective, multicentre cohort study. Lancet Oncol. 2013;14(7):609-618.

26. Straver ME, Loo CE, Alderliesten T, Rutgers EJ, Vrancken Peeters MT. Marking the axilla with radioactive iodine seeds (MARI procedure) may reduce the need for axillary dissection after neoadjuvant chemotherapy for breast cancer. Br J Surg. 2010;97(8):1226-1231.

27. Donker M, Straver ME, Wesseling J, et al. Marking axillary lymph nodes with radioactive iodine seeds for axillary staging after neoadjuvant systemic treatment in breast cancer patients: the MARI procedure. Ann Surg. 2015;261(2):378-382.

28. Koolen BB, Donker M, Straver ME, et al. Combined PET-CT and axillary lymph node marking with radioactive iodine seeds (MARI procedure) for tailored axillary treatment in node-positive breast cancer after neoadjuvant therapy. Br J Surg. 2017;104(9):1188-1196.

29. van der Noordaa MEM, van Duijnhoven FH, Straver ME, et al. Major Reduction in Axillary Lymph Node Dissections After Neoadjuvant Systemic Therapy for Node-Positive Breast Cancer by combining PET/CT and the MARI Procedure. Ann Surg Oncol. 2018;25(6):1512-1520.

30. Caudle AS, Yang WT, Krishnamurthy S, et al. Improved Axillary Evaluation Following Neoadjuvant Therapy for Patients With Node-Positive Breast Cancer Using Selective Evaluation of Clipped Nodes: Implementation of Targeted Axillary Dissection. J Clin Oncol. 2016;34(10):1072-1078. 




\section{PART I}

De-escalation of axillary management after neoadjuvant systemic therapy 



\section{CHAPTER}

De-escalation of axillary surgery in breast cancer patients treated in the neoadjuvant setting: a Dutch population-based study

JM Simons

LB Kloppert

EJT Luiten

CC van der Pol

S Samiei

HJH de Wit

$S$ Siesling

ML Smidt 


\section{ABSTRACT}

\section{Background}

An overall trend is observed towards de-escalation of axillary surgery in patients with breast cancer. The objective of this study was to evaluate this trend in patients treated with neoadjuvant systemic therapy (NST).

\section{Methods}

patients with cT1-4N0-3 breast cancer treated with NST (2006-2016), were selected from the Netherlands Cancer Registry. Patients were classified by clinical node status (cN) and type of axillary surgery. Uni- and multivariable logistic regression analysis were performed to determine clinicopathological factors associated with performing ALND in cN+ patients.

\section{Results}

A total of 12461 patients treated with NST were identified (5830 cNO patients (46.8\%), $6631 \mathrm{cN}+$ patients $(53.2 \%))$. In cNO patients, an overall increase in sentinel lymph node biopsy (SLNB) only (not followed by ALND) was seen from 11\% in 2006 to $94 \%$ in 2016. SLNB performed post-NST increased from $33 \%$ to $62 \%$. In $\mathrm{cN}+$ patients, an overall decrease in ALND was seen from $99 \%$ in 2006 to $53 \%$ in 2016. Age (OR 1.01, $\mathrm{Cl} 1.00-1.02$ ), year of diagnosis (OR $0.47, \mathrm{Cl} 0.44-0.50$ ), HER2 positive disease (OR 0.62, $\mathrm{Cl}$ 0.52-0.75), clinical tumor stage (T2 vs. T1 OR 1.32, Cl 1.06-1.65, T3 vs. T1 OR 2.04, $\mathrm{Cl} 1.58-2.63, \mathrm{~T} 4$ vs. T1 OR $6.37, \mathrm{Cl} 4.26-9.50$ ) and clinical nodal stage (N3 vs. N1 OR $1.65, \mathrm{Cl} 1.28-2.12$ ), were correlated with performing ALND in $\mathrm{cN}+$ patients.

\section{Conclusions}

ALND decreased substantially over the past decade in patients treated with NST. Assessment of long-term prognosis of patients in whom ALND is omitted after NST is urgently needed. 


\section{INTRODUCTION}

In breast cancer, systemic therapy is increasingly administered in the neoadjuvant setting (i.e. neoadjuvant systemic therapy (NST)). ${ }^{1,2}$ One of the advantages of NST is the possibility of downsizing or even downstaging disease, which can occur in breast and/or axilla. In the best-case scenario, patients achieve a pathologic complete response ( $p C R)$, meaning that there is no histologic evidence of residual tumor. Downstaging is not only associated with a favorable prognosis, it also enables surgeons to opt for less extensive surgery after NST.

In clinically node negative (cNO) patients, sentinel lymph node biopsy (SLNB) is widely accepted as primary regional staging procedure. In the case of a positive SLNB with limited tumor burden it is safe to omit completion axillary lymph node dissection (ALND) in patients treated with lumpectomy in terms of disease-free and overall survival. ${ }^{3-7}$ In the AMAROS trial, CT1-2NO patients with a positive SLNB and treated with lumpectomy or mastectomy, were randomly assigned to completion ALND or axillary radiotherapy. ${ }^{8}$ The 5-year axillary recurrence rate was comparable in both groups, but measurable lymphedema occurred significantly less frequently in the axillary radiotherapy-arm. The results of these trials resulted in decreasing use of ALND, as was proven by several cohort studies over the past years. ${ }^{9-12}$ In these studies, patients treated with NST were not included and it is yet unknown if this decrease in axillary surgery also affects cNO patients who were treated with NST.

In clinically node positive $(\mathrm{cN}+)$ patients, axillary staging is an area of controversy. Traditionally, ALND was performed in all patients. However, at least 1 out of $3 \mathrm{cN}+$ patients treated with NST, converts to a pathological node negative axilla. ${ }^{13}$ Since $\mathrm{CN}+$ patients with an axillary pCR are not expected to benefit from ALND, different less invasive methods have been proposed to replace ALND. Such as SLNB, MARI (marking the axillary positive lymph node with an iodine seed) and Targeted Axillary Dissection (i.e. a combination of the SLNB and a MARI-like procedure). However, since sufficient data are lacking on the outcome of $\mathrm{cN}+$ patients in whom ALND is omitted after NST, broad implementation of less invasive staging procedures in clinical practice may be hampered.

With the increased use of NST in both cNO and cN+ patients, and the de-escalation of axillary surgery in patients treated in the adjuvant setting, it is hypothesized that a trend of de-escalation will also be noticed in patients treated with NST. Therefore, the aim of this study was to evaluate trends in de-escalation of axillary surgery in cT1-4NO-3 breast cancer patients treated with NST in the Netherlands. 


\section{MATERIALS AND METHODS}

Data were collected from the Netherlands Cancer Registry (NCR). The NCR is hosted by the Netherlands Comprehensive Cancer Organisation (IKNL). Specially trained registration clerks gather data directly form the patient files in all hospitals in the Netherlands. Patients with cT1-4N0-3 breast cancer treated with NST, between 2006 and 2016, were included. Patients with occult breast cancer were also included. For each patient, the following variables were documented: hospital type (academic, teaching, community), age, morphological subtype, receptor status, TNM status prior to and after NST, NST regimens, type of breast and axillary surgery, axillary pCR, and adjuvant treatment plans. Axillary pCR was defined as the absence of residual axillary disease in all examined lymph nodes independent of the type of axillary surgery. Isolated tumor cells were included in the definition of axillary pCR. Patients were excluded if no lymph nodes were identified during surgery or if the number of positive lymph nodes was unknown. Patients with unknown cN status, distant metastasis or patients in whom surgery of the breast was not performed were also excluded.

\section{Patients}

In the Netherlands, the axilla is generally assessed by means of ultrasound at the time of diagnosis. In the case of suspicious lymph nodes, either fine needle aspiration (FNAC) or core needle biopsy (CNB) is performed to assess the presence of metastasis. A cNO status was defined as the absence of, suspicious lymph nodes on axillary ultrasound or the absence of a positive result on FNAC/CNB when performed. A CN+ status was defined as the presence of suspicious lymph nodes on axillary ultrasound and/or in combination with pathologically confirmed metastasis by FNAC/CNB. For cNO patients, the following subgroups regarding type of axillary surgerywere documented: SLNB only (i.e. not followed by completion ALND), SLNB followed by completion ALND, and ALND (not preceded by SLNB). Patients in whom the timing of SLNB was unknown were excluded from analysis. For cNO patients in whom SLNB was performed prior to NST, data on ypN status were only available when an ALND was performed. For $\mathrm{cN}+$ patients, the following subgroups were documented: SLNB and/or MARI only (i.e. not followed by completion ALND), SLNB and/or MARI followed by completion ALND and ALND (not preceded by SLNB and/or MARI). MARI includes any procedure in which the pathologically confirmed positive lymph node was marked prior to NST and selectively removed after NST.

When referred to ypN status, this is always based on pathologic examination of lymph nodes and not on post-NST clinical examination of the axilla. 


\section{Statistical analysis}

Descriptive statistics were performed to evaluate trends over time for omission of ALND in the overall population, in $\mathrm{cNO}$ and $\mathrm{cN}+$ patients. In addition, potential differences were explored in the axillary management associated with the type of hospital where patients were treated. Univariable and multivariable logistic regression analysis was performed to determine clinicopathological factors associated with performing ALND in $\mathrm{cN}+$ patients. Odds ratios (ORs) were presented with $95 \%$ confidence intervals ( $\mathrm{Cls}$ ). Two-sided $p$-values of $<0.05$ were considered statistically significant. Data analysis was performed using Stata/SE Statistical Software for Windows, version 14.2 (College Station, TX: StataCorp LP).

\section{RESULTS}

A total of 15725 breast tumors treated with NST (10\% of all breast tumors) were identified between January 2006 and December 2016 and registered in the NCR. Cases were excluded for the following reasons: distant metastasis $(n=683)$, treated with neoadjuvant radiotherapy $(n=21)$, unknown $\mathrm{cN}$ status $(n=165)$, unknown cT status $(n=136)$, unknown hospital type $(n=1)$, no breast surgery $(n=838)$, unknown type of axillary surgery $(n=674)$, unknown outcome of axillary surgery $(n=193)$ and unknown timing of SLNB ( $n=553)$. Altogether, 12.461 breast tumors were included for the final analysis. See Table 2.1 for clinicopathologic characteristics. In 7106 of 12461 (57\%) cases, ALND was performed. From 2006 to 2016, an overall decrease in the rate of ALND was observed from $96 \%$ to $29 \%$.

\section{cNO patients}

Nodal status was negative at the time of diagnosis (cNO) in 5830 cases (46.8\%). The proportion of cNO patients treated with NST increased from 35\% in 2006 to $50 \%$ in 2016. In total, 4301 (73.8\%) underwent SLNB only and 1529 (26.2\%) underwent ALND (+/- preceded by SLNB). From 2006 to 2016, the rate of SLNB only (not followed by ALND) increased from $11 \%$ to $94 \%$ (see Figure 2.1). The rate of ALND decreased in both patient groups with ypNO and ypN+ status (see Table 2.2). In the patients in whom SLNB was not followed by ALND, the proportion of patients with positive SLNs increased from $7 \%$ to $19 \%$.

SLNB was performed prior to compared with after NST in 3401 (65\%) and 1815 (35\%) cases, respectively. Over time, SLNB was increasingly performed after NST (33\% in 2006 vs. $62 \%$ in 2016). The overall rate of completion ALND was $23.4 \%$ when SLNB was performed prior to NST and $6.7 \%$ when SLNB was performed after NST $(p<0.001)$. For SLNB performed prior to NST, the rate of completion ALND decreased from $58 \%$ to 
$12 \%$, for SLNB performed after NST the rate of completion ALND decreased from $46 \%$ to $2 \%$ (see Figure 2.1 ).

Table 2.1 Clinicopathologic characteristics of all cT1-4N0-3 breast cancer patients treated with NST between 2006 and 2016 ( $n=12.461)$.

\begin{tabular}{|c|c|}
\hline Characteristics & $\mathbf{N}(\%)$ \\
\hline \multicolumn{2}{|l|}{ Year of diagnosis } \\
\hline $2006-2009$ & $2286(18.4)$ \\
\hline $2010-2013$ & $4712(37.8)$ \\
\hline $2014-2016$ & $5463(43.8)$ \\
\hline Age in years, median (range) & 50 (range $18-87$ ) \\
\hline \multicolumn{2}{|l|}{ Histologic subtype } \\
\hline Ductal & $9832(79)$ \\
\hline Lobular & $1256(10)$ \\
\hline Adenocarcinoma NOS & $550(4.4)$ \\
\hline Mixed ductal and lobular & $307(2.5)$ \\
\hline Other & $516(4.1)$ \\
\hline \multicolumn{2}{|l|}{ Receptor Status } \\
\hline HR-/HER2- & $1751(16.3)$ \\
\hline HR-/HER2+ & $987(9.2)$ \\
\hline $\mathrm{HR}+/ \mathrm{HER} 2+$ & $1756(16.4)$ \\
\hline HR+/HER2- & $6243(58.2)$ \\
\hline HR and/or HER2 status unknown & $1724(13.8)$ \\
\hline \multicolumn{2}{|l|}{ TNM status } \\
\hline TO - 1 - 2 - 3 - 4 & $14(0.1)-1647(13.2)-6595(53)-2696(21.6)-1509$ (12.1) \\
\hline NO - $1-2-3$ & $5830(47)-5610(45)-258(2)-760(8)$ \\
\hline $\mathrm{MO}-\mathrm{X}$ & $12351(99)-110(1)$ \\
\hline Lumpectomy & $5087(41)$ \\
\hline Mastectomy & $7374(59)$ \\
\hline \multicolumn{2}{|l|}{ Neoadjuvant systemic regimen } \\
\hline Chemotherapy only & $9382(75)$ \\
\hline Chemotherapy with HER2 therapy & $2732(22)$ \\
\hline Chemotherapy with endocrine therapy & $286(2)$ \\
\hline $\begin{array}{l}\text { Chemotherapy with HER2 and endocrine } \\
\text { therapy }\end{array}$ & $61(1)$ \\
\hline
\end{tabular}

NOS, not otherwise specified; HR, hormone receptor; NST, neoadjuvant systemic therapy.

Overall, 4288 of $5830 \mathrm{cNO}$ patients (74\%) were treated with adjuvant radiotherapy (80\% in 2006 and 68\% in 2016). Data on radiotherapy fields were unknown for the majority of cNO patients until 2010. From 2011 until 2016, data on radiotherapy fields were known for $91.7 \%$ (3244/3539) of cNO patients treated with adjuvant radiotherapy, ranging from $84 \%$ to $95.8 \%$ dependent on the year of diagnosis. Adjuvant radiotherapy included regional radiotherapy in $36 \%(122 / 339)$ of cNO patients treated with radiotherapy in 2011 and in 26\% (100/381) of cNO patients treated with radiotherapy in 2016. 
Figure 2.1 Course over time for axillary staging in cNO patients treated with NST.

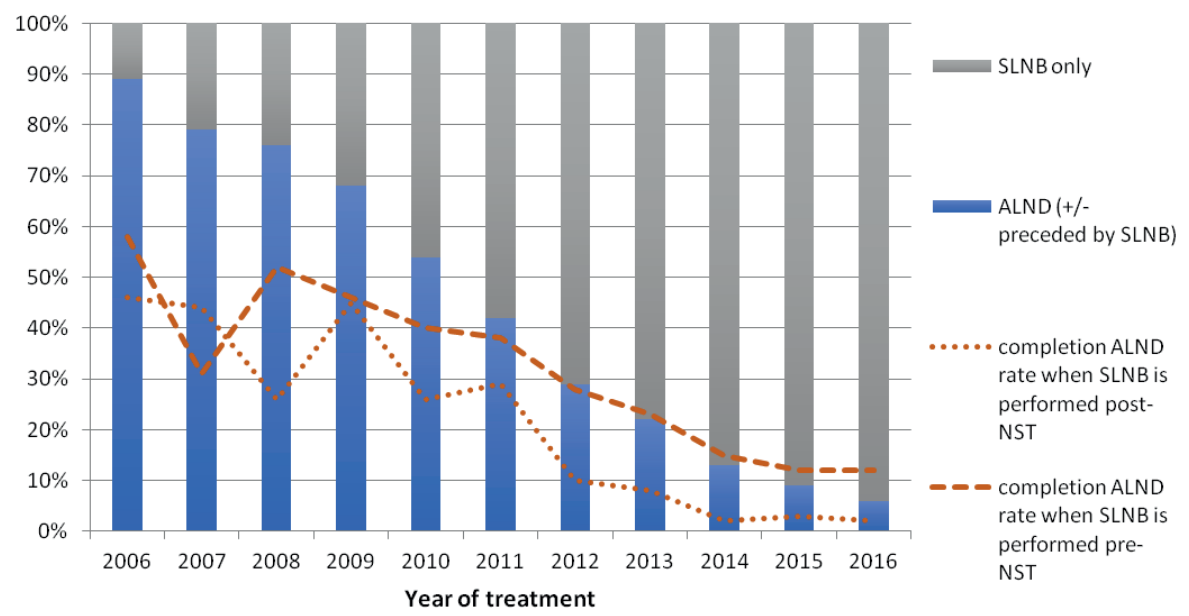

\section{$\mathrm{cN}+$ patients}

Nodal status was positive at the time of diagnosis ( $\mathrm{cN}+$ ) in 6631 patients $(53.2 \%)$. In total, 1054 (16\%) underwent SLNB and/or MARI only and $5577 \mathrm{cN}+$ patients (84\%) underwent ALND (+/- preceded by SLNB and/or MARI). From 2006 to 2016, the rate of SLNB and/or MARI only increased from $1 \%$ to $46 \%$ and the rate of (completion) ALND decreased from $99 \%$ to $54 \%$ (see Figure 2.2 ). Over this period, the rate of ALND decreased from $98 \%(55 / 56)$ to $42 \%(48 / 115)$ in cN+ypNO patients and from $100 \%$ to $56 \%(296 / 526)$ in cN+ypN+ patients (see Table 2.2). In 2016, $372 \mathrm{cN}+$ patients (58\%) underwent staging by SLNB and/or MARI: in 294 patients (79\%) this was not followed by ALND.

In multivariable logistic regression analysis, the following variables were associated with significantly decreased odds ratios for ALND: year of diagnosis (OR $0.47, \mathrm{Cl}$ $0.44-0.50$ ) and HER2 positive disease (OR $0.62, \mathrm{Cl} 0.52-0.75$ ). The following variables were associated with significantly increased odds ratios for ALND: age (OR 1.01, Cl 1.00-1.02), clinical tumor stage (T2 vs. T1 OR 1.32, Cl 1.06-1.65, T3 vs. T1 OR 2.04, Cl 1.58-2.63, T4 vs. T1 OR 6.37, Cl 4.26-9.50) and clinical nodal stage (N3 vs. N1 OR 1.65, Cl 1.28-2.12) (see Table 2.3). 


\begin{tabular}{|c|c|c|c|c|c|c|c|}
\hline 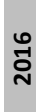 & $\mathbb{ర}$ & 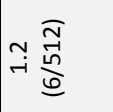 & 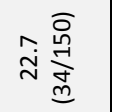 & 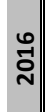 & $\tilde{్}$ & 守 & นึ่ \\
\hline$\stackrel{n}{\sim}$ & 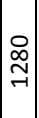 & 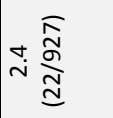 & 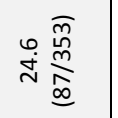 & กุ. & 只 & 강 & 今. \\
\hline$\stackrel{\square}{\sim}$ & gे & 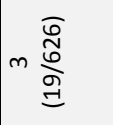 & 골 & पे & $\begin{array}{l}\bullet \\
\infty \\
\infty\end{array}$ & 남 & 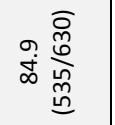 \\
\hline$\stackrel{m}{\stackrel{m}{\sim}}$ & $\overrightarrow{\tilde{m}}$ & 㐫 & 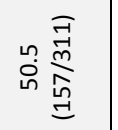 & ํ. & $\frac{i}{d}$ & 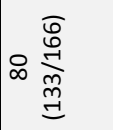 & 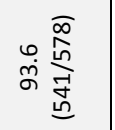 \\
\hline 고ำ & 영 & - & & సุำ & $\begin{array}{l}\infty \\
0 \\
0\end{array}$ & 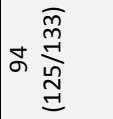 & $\underset{n}{\stackrel{n}{\frac{n}{+}}}$ \\
\hline ¿ั. & ָ̃ & 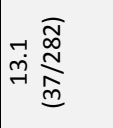 & 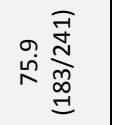 & :- & : & નું & 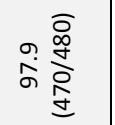 \\
\hline 오․ & m & 寉 & 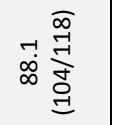 & مी & 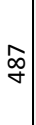 & 兽 & 의 $\underset{\substack{\frac{\infty}{m} \\
\stackrel{m}{m}}}{\stackrel{m}{n}}$ \\
\hline ఫ્े & $\stackrel{\substack{\infty \\
\infty \\
\sim}}{-1}$ & 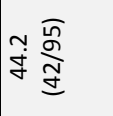 & స̃ & ठ্े & $\widehat{o}$ & $\stackrel{\substack{\infty \\
\hdashline}}{\underset{\infty}{\infty}}$ & مْ \\
\hline 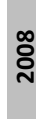 & 穴 & 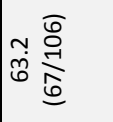 & 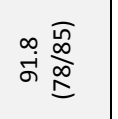 & $\stackrel{\infty}{0}$ & $\begin{array}{l}\stackrel{\wp}{q} \\
\stackrel{f}{q}\end{array}$ & 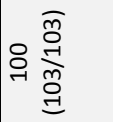 & 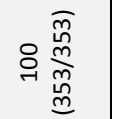 \\
\hline ఫิे & $\stackrel{\infty}{m}$ & 고 & ๙ู่ & ธ్సે| & $\begin{array}{l}m \\
\stackrel{m}{n}\end{array}$ & $\begin{array}{ll}\infty & \overline{0} \\
\infty & \infty \\
\infty & \stackrel{\infty}{L} \\
\sigma & \underline{\infty}\end{array}$ & o: $\underset{0}{\stackrel{\text { I }}{\stackrel{I}{I}}}$ \\
\hline ષั่ & $\mid \begin{array}{l}\dot{\Xi} \\
\stackrel{-1}{1}\end{array}$ & 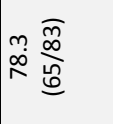 & ঃ & ¿્స & है & 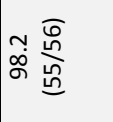 & 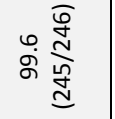 \\
\hline 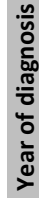 & $\left|\begin{array}{l|l}.0 \\
+ \\
0 \\
2 \\
2 \\
0 \\
2 \\
0\end{array}\right|$ & 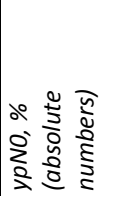 & 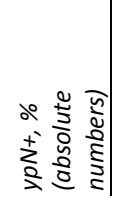 & 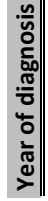 & 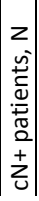 & 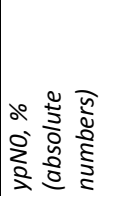 & 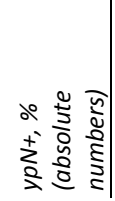 \\
\hline
\end{tabular}


Figure 2.2 Course over time for axillary staging in $\mathrm{cN}+$ patients treated with NST.

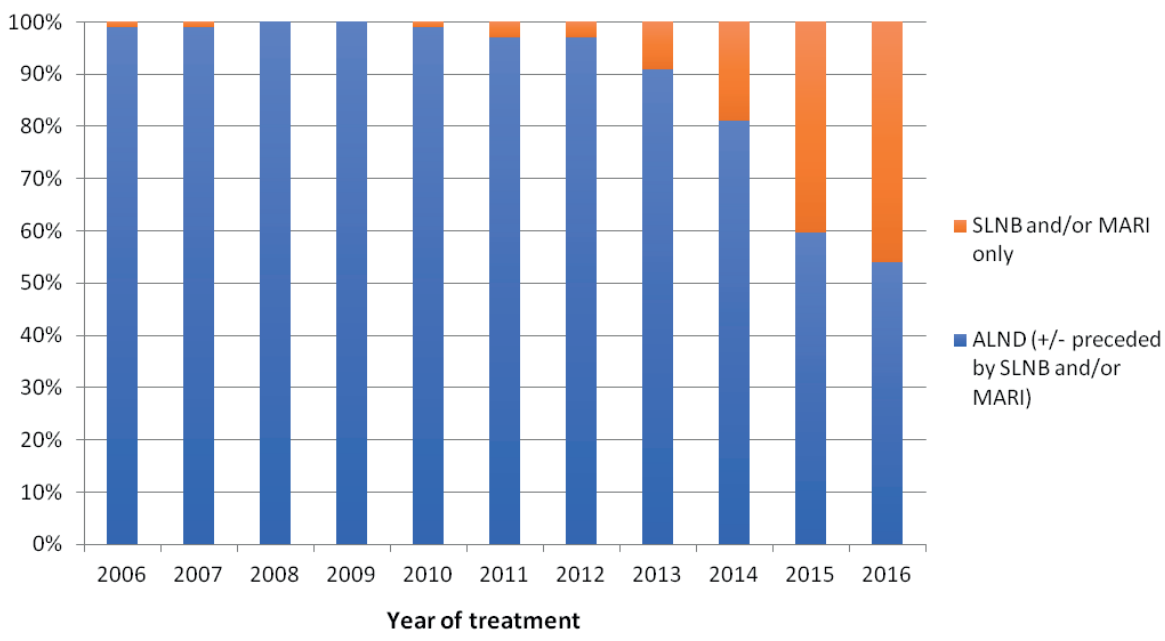

Table 2.3 Univariable and multivariable analysis for performing ALND in cT1-4N+ patients treated with NST.

\begin{tabular}{l||c|c|c|c}
\multicolumn{1}{c||}{} & \multicolumn{2}{c}{ Univariable Analysis } & \multicolumn{2}{c}{ Multivariable Analysis } \\
\cline { 2 - 5 } \multicolumn{1}{l|}{} & $\mathrm{OR}(95 \% \mathrm{Cl})$ & $\mathrm{P}$ value & $\mathrm{OR}(95 \% \mathrm{Cl})$ & $\mathrm{P}$ value \\
\hline \hline Year of diagnosis & $0.48(0.45-0.50)$ & $\mathrm{P}<.001$ & $0.47(0.44-0.50)$ & $\mathrm{P}<.001$ \\
\hline Age (per year) & $1.01(0.99-1.01)$ & $\mathrm{P}=.051$ & $1.01(1.00-1.02)$ & $\mathrm{P}=.042$ \\
\hline Clinical tumor status & & & & \\
T1 & Reference & & Reference & \\
T2 & $1.36(1.14-1.63)$ & $\mathrm{P}=.001$ & $1.32(1.06-1.65)$ & $\mathrm{P}=.013$ \\
T3 & $2.09(1.69-2.58)$ & $\mathrm{P}<.001$ & $2.04(1.58-2.63)$ & $\mathrm{P}<.001$ \\
T4 & $9.56(6.70-13.63)$ & $\mathrm{P}<.001$ & $6.37(4.26-9.50)$ & $\mathrm{P}<.001$ \\
\hline Clinical node status & & & & \\
N1 & Reference & & Reference & \\
N2 & $1.12(0.79-1.60)$ & $\mathrm{P}=.531$ & $1.33(0.88-2.01)$ & $\mathrm{P}=.176$ \\
N3 & $1.23(0.98-1.53)$ & $\mathrm{P}=.070$ & $1.65(1.28-2.12)$ & $\mathrm{P}<.001$ \\
\hline ER positive & $0.97(0.84-1.12)$ & $\mathrm{P}=.703$ & $1.08(0.91-1.29)$ & $\mathrm{P}=.358$ \\
\hline HER2 positive & $0.83(0.72-0.96)$ & $\mathrm{P}=.013$ & $0.62(0.52-0.75)$ & $\mathrm{P}<.001$ \\
\hline
\end{tabular}

Overall, 5.824 out of $6631 \mathrm{cN}+$ patients (88\%) were treated with adjuvant radiotherapy. Data on radiotherapy fields were unknown for the majority of $\mathrm{cN}+$ patients until 2010. From 2011 until 2016, data on radiotherapy fields were known for $91.9 \%(3671 / 3996)$ of $\mathrm{cN}+$ patients treated with adjuvant radiotherapy, ranging from $83.6 \%$ to $97 \%$ dependent on the year of diagnosis. Adjuvant radiotherapy included regional radiotherapy in $67.8 \%$ (318/469) of $\mathrm{cN}+$ patients treated with radiotherapy in 2011 and in $80 \%$ (368/458) of cN+ patients treated with radiotherapy in 2016. 


\section{Trends by hospital type}

Overall, 823 (6.6\%), 6457 (51.8\%), and 5181 (41.6\%) patients were treated in academic hospitals, teaching hospitals, and community hospitals, respectively. From 2006 to 2016, an overall decrease in ALND was observed from $97 \%$ to $38 \%$ in academic hospitals, from $94 \%$ to $27 \%$ in teaching hospitals and from $97 \%$ to $32 \%$ in community hospitals. In 2016, staging was performed by means of SLNB and/or MARI in $53 \%(16 / 30)$ of $\mathrm{cN}+$ patients in academic hospitals, $61 \%(216 / 354)$ in teaching hospitals, and $52 \%(140 / 268)$ in community hospitals $(p=.083)$. In these patients, SLNB and/or MARI was not followed by ALND in 75\% (12/16), 75\% (161/216) and 86\% $(121 / 140)$, respectively $(p=.025)$.

\section{DISCUSSION}

This large Dutch population-based cohort study showed that axillary surgery has changed considerably in daily practice over the past decade in CT1-4N0-3 breast cancer patients treated with NST. In cNO patients a substantial decrease in ALND and increase in SLNB only was observed, with SLNB being increasingly performed after NST. Additionally, this study revealed that ALND has increasingly been omitted after NST in cN+ patients.

In patients who undergo adjuvant systemic therapy, indications for omitting ALND in cNO patients have extended over the years from patients with negative SLNS(s) to patients with positive SLN(s). ${ }^{14}$ Even ten-year survival outcomes from the ACOSOG Z0011 and IBCSG 23-01 trials corroborated non-inferiority of SLNB alone compared to ALND for patients with limited positive $\operatorname{SLN}(\mathrm{s}) .{ }^{4,15}$ Abandoning completion ALND in cNO patients with positive SLN(s) treated with adjuvant systemic therapy is already ongoing for years, even prior to the publication of the abovementioned trials. ${ }^{16-19}$ Ever since the publication of these trial results, implementation of SLNB-only in this population is expanding. ${ }^{11,20}$ The current study shows that abandoning completion ALND in cNO patients with positive $\operatorname{SLN}(\mathrm{s})$ is also taking place in patients treated with NST, eventhough results of the previous trials only apply to patients treated with adjuvant systemic therapy. Over the past decade, SLNB was increasingly performed after NST rather than prior to NST. Several previous studies demonstrated that performing SLNB after NST is associated with lower rates of a positive SLNB. ${ }^{21-23}$ Thus, ALND can be omitted more often when SLNB is performed after NST. Hence, the change in timing of SLNB found in this study contributed to the decreasing use of ALND.

The results presented here prove that clinicians are willing to adopt a SLNB-only strategy in the neoadjuvant setting as well, even though such a strategy is not 
evidence-based. Whether SLNB alone instead of the routine use of ALND in patients with limited positive SLN(s) treated in the neoadjuvant rather than adjuvant setting provides similar results in terms of overall survival is yet unknown. One should bear in mind that in contrast to the adjuvant setting, positive $\operatorname{SLN}(\mathrm{s})$ in the neoadjuvant setting indicates therapy-resistant disease and may represent a different tumor biology. Since long-term follow up of cNO patients with positive SLNs that did not undergo completion ALND in the neoadjuvant setting is lacking, it is too early to tell whether it is safe to consider and treat these groups similarly. Furthermore, it is unknown to what extent ALND has been replaced by regional radiotherapy in these patients.

Regarding $\mathrm{cN}+$ patients treated with NST, several less invasive procedures have been proposed over the past years in an attempt to prevent unnecessary ALND in patients who achieve axillary pCR: SLNB, excision of a pre-treatment marked positive lymph node (like MARI) or a combination of these two procedures (like Targeted Axillary Dissection and RISAS). ${ }^{13,24-26}$ Despite limited evidence for the safety of replacing ALND by these procedures, implementation of such strategies is occurring worldwide. ${ }^{27}$ The results of the current study confirm this trend: from 2013 (prior to publications of MARI and TAD), rates of SLNB and/or MARI for axillary staging after NST started increasing up to $58 \%$ in 2016. The increase in rates of SLNB and/or MARI in cN+ patients was present in all three types of hospitals. Although SLNB and/or MARI is offered more and more to $\mathrm{CN}+$ patients in order to omit ALND in case of axillary pCR, $42 \%$ of patients with an axillary PCR still underwent (completion) ALND in 2016. This indicates that selecting the right patient for the appropriate procedure is challenging. At the same time, completion ALND may have been performed as part of validating studies. In some institutions, ALND is performed in all $\mathrm{cN}+$ patients, irrespective of response to NST, which may also in part explain this finding. Again, it is unknown to what extent ALND has been replaced by regional radiotherapy.

Notably, the decrease in ALND rates in $\mathrm{CN}+$ patients treated with NST is also present in patients who do not achieve an axillary pCR. In 2016, 44\% of the $\mathrm{cN}+$ patients with post-NST residual axillary disease, did not undergo completion ALND. Multiple studies reported improved survival for patients with a complete response (ypTO/ypNO) to NST compared to patients without a complete response. ${ }^{28,29}$ These results indicate the necessity of treatment escalation rather than de-escalation in patients without axillary pCR to improve prognosis, especially in certain subtypes (such as triple negative breast cancer). ${ }^{30}$ Whether current practices of omitting ALND in $\mathrm{cN}+$ patients without axillary pCR will negatively impact prognosis is yet unknown. In the Alliance 11202 trial, prognosis of $\mathrm{cN}+$ patients with positive $\mathrm{SLN}(\mathrm{s})$ after NST is compared between those treated with completion ALND and those treated with axillary radiotherapy. ${ }^{31}$ 
Although the current study describes a large cohort of patients, several limitations have to be taken into account. Regarding adjuvant treatment plans, sufficient data on radiotherapy fields were not available for the whole cohort. It is expected that simultaneously with the decreasing rates of ALND, rates of regional radiotherapy increase. This study did suggest such a trend in $\mathrm{cN}+$ patients, but further research is needed for a thorough assessment of (regional) radiotherapy administration in patients treated with NST. Furthermore, the impact of omitting ALND on prognosis in terms of overall and disease-free survival could not be assessed since data on recurrences are not (yet) available for this cohort.

To conclude, axillary surgical staging changed significantly with a major decrease in ALND rates in breast cancer patients treated with NST over the past decade. However, selecting the right patients for whom omitting ALND is oncologic safe appears challenging, especially in pre-treatment $\mathrm{cN}+$ patients and patients with residual axillary disease. Studies assessing long-term prognosis of such patients in whom ALND is omitted are urgently needed.

\section{ACKNOWLEDGMENTS}

The authors thank the registration clercks and the staff for gathering the data for the NCR and L. de Munck and S.J. Hogewoning for assistance with statistical analysis. 


\section{REFERENCES}

1. Mougalian SS, Soulos PR, Killelea BK, et al. Use of neoadjuvant chemotherapy for patients with stage I to III breast cancer in the United States. Cancer. 2015;121(15):2544-2552.

2. Vugts G, Maaskant-Braat AJ, Nieuwenhuijzen GA, Roumen RM, Luiten EJ, Voogd AC. Patterns of care in the administration of neo-adjuvant chemotherapy for breast cancer. A population-based study. Breast J. 2016;22(3):316-321.

3. Giuliano $\mathrm{AE}$, Ballman $\mathrm{K}, \mathrm{McC}$ all $\mathrm{L}$, et al. Locoregional recurrence after sentinel lymph node dissection with or without axillary dissection in patients with sentinel lymph node metastases: Long-term followup from the American College of Surgeons Oncology Group (Alliance) ACOSOG Z0011 randomized trial. Ann Surg. 2016;264(3):413-420.

4. Giuliano $A E$, Ballman KV, McCall L, et al. Effect of axillary dissection vs no axillary dissection on 10year overall survival among women with invasive breast cancer and sentinel node metastasis: The ACOSOG Z0011 (Alliance) randomized clinical trial. JAMA. 2017;318(10):918-926.

5. Giuliano $\mathrm{AE}$, Hunt $\mathrm{KK}$, Ballman KV, et al. Axillary dissection vs no axillary dissection in women with invasive breast cancer and sentinel node metastasis: a randomized clinical trial. JAMA. 2011;305(6):569-575.

6. Giuliano $A E, M c C a l l ~ L$, Beitsch $P$, et al. Locoregional recurrence after sentinel lymph node dissection with or without axillary dissection in patients with sentinel lymph node metastases: the American College of Surgeons Oncology Group Z0011 randomized trial. Ann Surg. 2010;252(3):426-432; discussion 432-423.

7. Galimberti V, Cole BF, Zurrida S, et al. Axillary dissection versus no axillary dissection in patients with sentinel-node micrometastases (IBCSG 23-01): a phase 3 randomised controlled trial. Lancet Oncol. 2013;14(4):297-305.

8. Donker M, van Tienhoven G, Straver ME, et al. Radiotherapy or surgery of the axilla after a positive sentinel node in breast cancer (EORTC 10981-22023 AMAROS): a randomised, multicentre, openlabel, phase 3 non-inferiority trial. Lancet Oncol. 2014;15(12):1303-1310.

9. Dengel LT, Van Zee KJ, King TA, et al. Axillary dissection can be avoided in the majority of clinically node-negative patients undergoing breast-conserving therapy. Ann Surg Oncol. 2014;21(1):22-27.

10. Mamtani A, Patil S, Van Zee KJ, et al. Age and receptor status do not indicate the need for axillary dissection in patients with sentinel lymph node metastases. Ann Surg Oncol. 2016;23(11):3481-3486.

11. Poodt IGM, Spronk PER, Vugts G, et al. Trends on axillary surgery in nondistant metastatic breast cancer patients treated between 2011 and 2015: A Dutch population-based study in the ACOSOGZ0011 and AMAROS era. Ann Surg. 2018;268(6):1084-1090.

12. Tsao MW, Cornacchi SD, Hodgson N, et al. A Population-based study of the effects of a regional guideline for completion axillary lymph node dissection on axillary surgery in patients with breast cancer. Ann Surg Oncol. 2016;23(10):3354-3364.

13. van Nijnatten TJ, Schipper RJ, Lobbes MB, Nelemans PJ, Beets-Tan RG, Smidt ML. The diagnostic performance of sentinel lymph node biopsy in pathologically confirmed node positive breast cancer patients after neoadjuvant systemic therapy: A systematic review and meta-analysis. Eur J Surg Oncol. 2015;41(10):1278-1287.

14. Francissen CM, Dings PJ, van Dalen T, Strobbe LJ, van Laarhoven HW, de Wilt JH. Axillary recurrence after a tumor-positive sentinel lymph node biopsy without axillary treatment: a review of the literature. Ann Surg Oncol. 2012;19(13):4140-4149.

15. Galimberti V, Cole BF, Viale G, et al. Axillary dissection versus no axillary dissection in patients with breast cancer and sentinel-node micrometastases (IBCSG 23-01): 10-year follow-up of a randomised, controlled phase 3 trial. Lancet Oncol. 2018;19(10):1385-1393.

16. Bilimoria KY, Bentrem DJ, Hansen NM, et al. Comparison of sentinel lymph node biopsy alone and completion axillary lymph node dissection for node-positive breast cancer. $J$ Clin Oncol. 2009;27(18):2946-2953.

17. Boughey JC. Axillary dissection can be avoided in the majority of clinically node-negative patients undergoing breast-conserving therapy, by Dengel et al. Ann Surg Oncol. 2014;21(1):8-10. 
18. Yi M, Giordano SH, Meric-Bernstam F, et al. Trends in and outcomes from sentinel lymph node biopsy (SLNB) alone vs. SLNB with axillary lymph node dissection for node-positive breast cancer patients: experience from the SEER database. Ann Surg Oncol. 2010;17 Suppl 3:343-351.

19. Hieken TJ, Boughey JC. Axillary dissection versus no axillary dissection in patients with sentinel-node micrometastases: commentary on the IBCSG 23-01 Trial. Gland Surg. 2013;2(3):128-132.

20. Weiss A, Mittendorf EA, DeSnyder SM, et al. Expanding implementation of ACOSOG Z0011 in surgeon practice. Clin Breast Cancer. 2018;18(4):276-281.

21. Hunt KK, Yi M, Mittendorf EA, et al. Sentinel lymph node surgery after neoadjuvant chemotherapy is accurate and reduces the need for axillary dissection in breast cancer patients. Ann Surg. 2009;250(4):558-566.

22. Fontein DB, van de Water W, Mieog JS, Liefers GJ, van de Velde CJ. Timing of the sentinel lymph node biopsy in breast cancer patients receiving neoadjuvant therapy - recommendations for clinical guidance. Eur J Surg Oncol. 2013;39(5):417-424.

23. van der Heiden-van der Loo M, de Munck L, Sonke GS, et al. Population based study on sentinel node biopsy before or after neoadjuvant chemotherapy in clinically node negative breast cancer patients: Identification rate and influence on axillary treatment. Eur J Cancer. 2015;51(8):915-921.

24. Caudle AS, Yang WT, Krishnamurthy S, et al. Improved axillary evaluation following neoadjuvant therapy for patients with node-positive breast cancer using selective evaluation of clipped nodes: implementation of targeted axillary dissection. J Clin Oncol. 2016;34(10):1072-1078.

25. Donker M, Straver ME, Wesseling J, et al. Marking axillary lymph nodes with radioactive iodine seeds for axillary staging after neoadjuvant systemic treatment in breast cancer patients: the MARI procedure. Ann Surg. 2015;261(2):378-382.

26. van Nijnatten TJA, Simons JM, Smidt ML, et al. A Novel less-invasive approach for axillary staging after neoadjuvant chemotherapy in patients with axillary node-positive breast cancer by combining radioactive iodine seed localization in the axilla with the sentinel node procedure (RISAS): A Dutch prospective multicenter validation study. Clin Breast Cancer. 2017;17(5):399-402.

27. Diego EJ, McAuliffe PF, Soran A, et al. Axillary staging after neoadjuvant chemotherapy for breast cancer: A pilot study combining sentinel lymph node biopsy with radioactive seed localization of pretreatment positive axillary lymph nodes. Ann Surg Oncol. 2016;23(5):1549-1553.

28. Cortazar $\mathrm{P}$, Zhang $\mathrm{L}$, Untch $\mathrm{M}$, et al. Pathological complete response and long-term clinical benefit in breast cancer: the CTNeoBC pooled analysis. Lancet. 2014;384(9938):164-172.

29. Mougalian SS, Hernandez M, Lei X, et al. Ten-year outcomes of patients with breast cancer with cytologically confirmed axillary lymph node metastases and pathologic complete response after primary systemic chemotherapy. JAMA Oncol. 2016;2(4):508-516.

30. Yang TJ, Morrow M, Modi S, et al. The effect of molecular subtype and residual disease on locoregional recurrence in breast cancer patients treated with neoadjuvant chemotherapy and postmastectomy radiation. Ann Surg Oncol. 2015;22 Suppl 3:S495-501.

31. NCT01901094. Comparison of axillary lymph node dissection with axillary radiation for patients with node-positive breast cancer treated with chemotherapy. Principal investigator: Judy Boughey. Mayo Clinic. Available at: https://clinicaltrials.gov/ct2/show/NCT01901094. Accessed November 5, 2018. 




\section{CHAPTER}

Patterns of axillary staging and management in clinically node positive breast cancer patients treated with neoadjuvant systemic therapy: results of a survey amongst breast cancer specialists

JM Simons AJG Maaskant-Braat

EJT Luiten MHK Leidenius TJA van Nijnatten

PG Boelens

LB Koppert

CC van der Pol $\mathrm{CJH}$ van de Velde

RA Audisio

ML Smidt

Eur J Surg Oncol. 2019 Aug. Doi: 10.1016/j.ejso.2019.08.012 


\section{ABSTRACT}

\section{Introduction}

Various options for axillary staging after neoadjuvant systemic therapy (NST) are available for breast cancer patients with a clinically positive axillary node $(\mathrm{cN}+)$. This survey assessed current practices amongst breast cancer specialists.

\section{Materials and methods}

A survey was performed amongst members of the European Society of Surgical Oncology and two UK-based Associations: the Association of Breast Surgery and the British Association of Surgical Oncology. The survey included 3 parts: 1 . general information, 2. diagnostic work-up and 3. axillary staging after NST.

\section{Results}

A total of 310 responses were collected: parts 1, 2 and 3 were fully completed by 282 (91\%), $270(87.1 \%)$ and $225(72.6 \%)$ respondents respectively. After NST, 153/267 (57.3\%) respondents currently perform ALND routinely and 114 (42.7\%) respondents perform less invasive restaging of the axilla with possible omission of ALND. In the latter group, $85 \%$ does and $15 \%$ does not use nodal response seen on imaging to guide the axillary restaging procedure. Regarding respondents that do use imaging: 95\% would perform a less invasive staging procedure in case of complete nodal response on imaging (63\% sentinel lymph node biopsy (SLNB), excision of a previously marked positive node with SLNB (21\%) and without SLNB (11\%)). In case of no nodal response on imaging $77 \%$ would perform ALND.

\section{Conclusion}

Current axillary staging and management practices in $\mathrm{cN}+$ patients after NST vary widely. To determine optimal axillary staging and management in terms of quality of life and oncologic safety, breast specialists are encouraged to include patients in clinical trials/prospective registries. 


\section{INTRODUCTION}

The management of the axilla in clinically node-negative breast cancer patients has evolved towards a less or even non-invasive approach. For clinically node-positive breast cancer patients $(\mathrm{cN}+)$ however, the axillary lymph node dissection (ALND) has been the standard of care until recently. $\mathrm{cN}+$ patients are increasingly receiving systemic therapy before surgery (i.e. neoadjuvant systemic therapy (NST)). As a result of NST, in at least $1 / 3$ of pre-treatment $c N+$ patients the axilla converts to nodenegative. ${ }^{1}$ In case of a pathologic complete response ( $\left.p C R\right)$ in the axilla, ALND may be unnecessary and its purpose is therefore being questioned. ${ }^{2,3}$

In order to identify cN+ypNO patients, several less invasive procedures have been proposed. These procedures can be largely divided into 3 groups: sentinel lymph node biopsy (SLNB), excision of a pre-treatment marked positive lymph node (e.g. MARI: Marking Axillary lymph node with Radioactive lodine seed) ${ }^{4}$ or procedures involving the combination of both (e.g. TAD: Targeted Axillary Dissection ${ }^{5}$, RISAS: Radioactive lodine Seed localization in the Axilla combined with a SLNB; the iodine seed is placed in the positive axillary lymph node prior to start of $\mathrm{NST}^{6}$ ). Currently, no consensus exists on which procedure is most accurate for axillary staging after NST. Moreover, long-term data on whether these less invasive procedures can safely replace ALND, even in case of axillary $\mathrm{PCR}$, are lacking.

The lack of evidence-based consensus guidelines for $\mathrm{cN}+$ patients treated with NST results into broadly differing patterns of care in the management of the axilla. The present survey focused on breast cancer specialists (mainly European) to assess current practices regarding diagnostic work up and axillary staging in $\mathrm{cN}+$ patients treated with NST.

\section{MATERIALS AND METHODS}

\section{Participants and survey}

An anonymous survey was made accessible to members of the European Society of Surgical Oncology, the Association of Breast Surgery (ABS) and the British Association of Surgical Oncology (BASO). A link to the survey was uploaded on the websites of these 3 Associations and included in several newsletters. The survey was developed using SurveyMonkey Inc. and consisted of 3 parts: 1 . general information, 2. diagnostic work-up/indications for NST and 3. axillary staging after NST. The expected time spent to complete the survey was approximately 5 to 10 minutes. The maximum number of questions was 38 . 


\section{Collection and analysis of responses}

Responses were collected from March to October 2018. Statistical analysis was performed using IBM SPSS Statistics for Windows, version 24 (IBM. Corp, Armonk, New York, USA). For each question, the number of respondents was determined. The selected answers to each question were reported as percentages of the total number of responses to each question. Subgroups were created for respondents that routinely performed ALND and respondents that selectively omitted ALND in $\mathrm{cN}+$ patients. To compare unpaired categorical variables the Chi-squared test was used. A $p$-value of $\leq 0.05$ was considered statistically significant.

\section{RESULTS}

\section{General information}

Characteristics regarding general information of the respondents are summarized in Table 3.1. A total of 310 respondents submitted the survey, of whom 282 (91\%) fully completed the first section on general information. All 310 respondents felt this topic to be clinically relevant. Institutional lack of consensus was reported by $113 / 310$ (36.5\%) respondents. Dissatisfaction with local protocols for axillary staging and management was reported by $94 / 310(30.3 \%)$ respondents as a consequence of the lack of consensus or protocols, performing ALND irrespective of response to NST, concerns regarding insufficient data on long-term oncologic safety, management differing per individual case and disagreement between specialties. Respondents experiencing lack of consensus in their institution were less often satisfied with current practices compared to respondents experiencing consensus: $46.9 \%$ vs. $82.7 \%$ respectively $(p<.001)$.

\section{Diagnostic work-up and indications for NST}

A total of $270 / 282$ (95.7\%) respondents fully completed the second section on diagnostic work-up and indications for NST. Axillary ultrasound was routinely performed to assess the axilla prior to start of NST by $87 \%$ of respondents. The main reasons not to perform axillary ultrasound were: a normal physical examination of the axilla or other imaging modalities were performed (i.e. PET-CT or MRI). The majority of respondents $(70.9 \%)$ did not perform other imaging examinations in addition to axillary ultrasound to determine the axillary tumour load prior to NST. The majority of 210 respondents $(74.5 \%)$ stated either FNAC or CNB was mandatory to confirm $\mathrm{cN}+$ disease, the remainder relied on physical examination and/or imaging. The main reasons not to perform FNAC or CNB were radiological signs of distant metastasis, 
patient age or very high clinical suspicion of axillary lymph node status (i.e. FNAC or CNB outcome would not change treatment plan).

Table 3.1 General information characteristics.

\begin{tabular}{|c|c|}
\hline Variable & Total responses $n=310(\%)$ \\
\hline Confirm Clinical relevance of topic & $310(100.0)$ \\
\hline Institutional lack of consensus & $113(36.7)$ \\
\hline Dissatisfaction with local protocols & $94(30.5)$ \\
\hline Variable & Total responses $n=282$ (\%) \\
\hline $\begin{array}{l}\text { Qualification } \\
\text { Surgical oncologist } \\
\text { Surgeon, participating in surgical oncology fellowship } \\
\text { Surgical resident } \\
\text { Other* } \\
\end{array}$ & $\begin{array}{l}230(81.5) \\
25(8.9) \\
12(4.3) \\
15(5.3) \\
\end{array}$ \\
\hline $\begin{array}{l}\text { Practicing in Continent } \\
\text { Europe } \\
\text { Asia } \\
\text { North America } \\
\text { South America } \\
\text { Africa }\end{array}$ & $\begin{array}{c}208(73.8) \\
44(15.6) \\
5(1.7) \\
11(3.9) \\
14(5.0) \\
\end{array}$ \\
\hline $\begin{array}{l}\text { Institution } \\
\text { University hospital } \\
\text { Breast cancer specific hospital } \\
\text { Large non-university teaching hospital } \\
\text { Community hospital } \\
\text { Other }\end{array}$ & $\begin{array}{l}125(44.3) \\
21(7.4) \\
79(28) \\
29(10.3) \\
28(10) \\
\end{array}$ \\
\hline $\begin{array}{l}\text { Years of experience as a specialist } \\
\leq 5 \\
6-10 \\
11-15 \\
\geq 16\end{array}$ & $\begin{array}{c}67(23.7) \\
58(20.6) \\
46(16.3) \\
111(39.4)\end{array}$ \\
\hline $\begin{array}{l}\text { Number of new breast cancer patients at institutional } \\
\text { level (yearly) } \\
\leq 500 \\
500-1000 \\
\geq 1000 \\
\text { Unknown/not sure }\end{array}$ & $\begin{array}{c}213(76.1) \\
45(16.1) \\
22(7.8) \\
2 \text { (not included in percentage calculation) }\end{array}$ \\
\hline $\begin{array}{l}\text { Percentage of patients treated with NST } \\
0-15 \% \\
16-30 \% \\
31-45 \% \\
46-60 \% \\
>61 \% \\
\text { Unknown/not sure } \\
\end{array}$ & $\begin{array}{c}112(41.7) \\
90(33.2) \\
27(10.0) \\
26(9.6) \\
16(5.9) \\
11 \text { (not included in percentage calculation) }\end{array}$ \\
\hline $\begin{array}{l}\text { On-site radiotherapy facility present } \\
\text { Yes } \\
\text { No }\end{array}$ & $\begin{array}{c}184(65.2) \\
98(34.8)\end{array}$ \\
\hline
\end{tabular}

*Other: $1 x$ medical oncologist, $1 x$ radiotherapist, $1 x$ final year trainee in breast surgery, $5 x$ general surgeon, $1 x$ plastic surgeon, $1 x$ pediatric surgeon, $1 x$ pharmacist, $2 x$ gynaecologist, $2 x$ associate specialist; \#Other: 12x comprehensive cancer center, 10x private hospital, $5 x$ district general hospital, $1 x$ maternity and gynaecology hospital. 
For $27.4 \%$ of respondents, $\mathrm{cN}+$ status was always an indication for NST in physically fit breast cancer patients aged $<70$ years and for $11 \%$ of respondents this was (almost) never the case. For the remaining respondents, the following factors influence whether NST was indicated in cN+ patients: histological subtype, molecular biology, $\mathrm{cN}$ status, size of primary tumour, tumour grade and patient preferences. The vast majority of respondents (79\%), indicated that gene expression profiling was (almost) never performed to advice for NST.

The majority of respondents (168/270; 62.2\%) never marked the pathologically positive lymph node prior to NST. One quarter breast specialists $(69 / 270 ; 25.6 \%)$ marked the positive lymph node as standard of care and 33/270 (12.2\%) only performed this as part of clinical trials. The majority of respondents who mark the positive lymph node prior to NST use a radiopaque clip (76/100; 76\%). Other methods to mark the lymph node prior to NST were iodine seed or injection of charcoal. Respondents who omitted ALND in $\mathrm{cN}+$ patients, marked lymph nodes more often than respondents who routinely performed ALND (50.8\% vs. $26.8 \%, p<.001)$.

\section{Axillary staging and treatment after NST}

A total of $225 / 270(83.3 \%)$ respondents fully completed the third and last section of the survey on axillary staging and treatment after NST. After NST, ALND was routinely performed by $153 / 267$ respondents $(57.3 \%)$. This rate was comparable between European respondents (56.3\%) and non-European respondents (59.7\%). The remaining 114/267 respondents performed less invasive restaging of the axilla with possible omission of ALND based on several clinicopathologic characteristics including; cN status prior to NST, number of positive lymph nodes prior to NST, age and presence of comorbidities (see Figure 3.1). Besides these characteristics, $85 \%$ rely on nodal response seen on imaging to choose the axillary staging procedure (see Figure 3.2). The nodal response was assessed (during and/or after NST) by means of ultrasound (88\%) and/or MRI (46.3\%) and/or PET/CT (22.2\%). In case of complete nodal response on imaging, 95\% (77/81) performed a less invasive axillary staging procedure, mainly SLNB (63\%). The remainder prefer SLNB combined with excision of a previously marked positive lymph node $(21 \%)$ or excision of a previously marked positive lymph node without SLNB (11\%). Five percent $(\mathrm{N}=4)$ of respondents did not perform any surgical staging procedure in patients with complete nodal response on imaging: 1 respondent opted for adjuvant radiotherapy, 2 respondents opted for a "wait and see" approach and 1 respondent stated this would depend on breast response and axillary tumour burden pre-NST. In case of partial or no nodal response on imaging, ALND was performed by the majority of respondents (59\% and $76 \%$ respectively). 
Figure 3.1 Less invasive axillary restaging with possible omission of ALND is performed in $\mathrm{cN}+$ patients dependent on (a combination of) the below characteristics.*

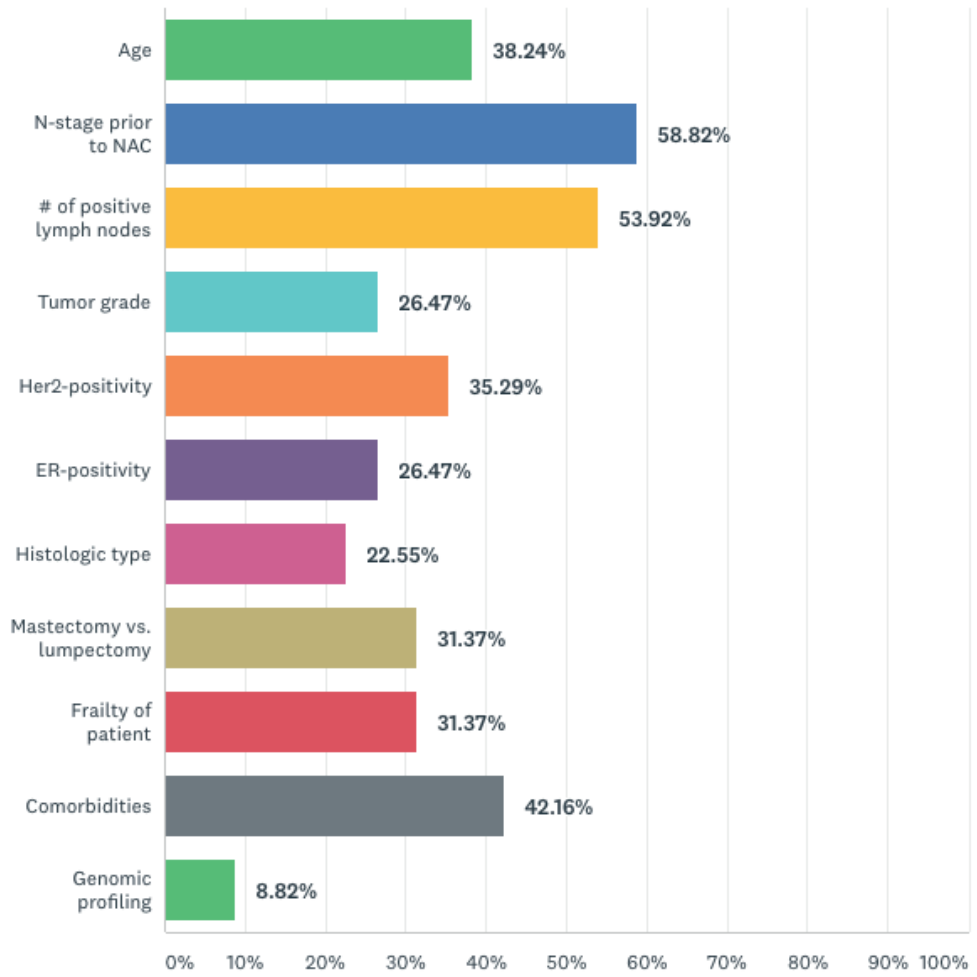

*114 respondents reported to perform less invasive restaging of the axilla with possible omission of ALND in $\mathrm{cN}+$ patients of whom 102 respondents reported on the above characteristics. Respondents could select multiple answers, therefore percentages do not add up to $100 \%$. Sixty-four percent of respondents selected 3 or more characteristics.

Several respondents (28/225) reported that the prescription of adjuvant radiotherapy and extension of fields also depended on factors other than pathologic response, like the number of suspicious or positive lymph nodes prior to NST. In case final pathologic assessment of lymph nodes showed axillary $\mathrm{pCR}, 70 \%$ of remaining respondents $(138 / 197)$ recommended no further axillary treatment (this rate was similar for respondents that did and did not routinely perform ALND (86/121 (71\%) and 52/76 (68\%) respectively, $p=.692$ ). In case final pathologic assessment of lymph nodes showed macrometastasis, $48 \%$ of respondents (93/194) recommended adjuvant regional radiotherapy (this rate was similar for respondents that did and did not routinely perform ALND (38/78 (48.7\%) and 55/116 (47.4\%) respectively, $p=.859)$. 
Figure 3.2 Type of axillary staging procedure based on the degree of nodal response seen on imaging (A: complete nodal response, B: Partial nodal response, C: no nodal response).

\section{A. Complete nodal response on imaging}

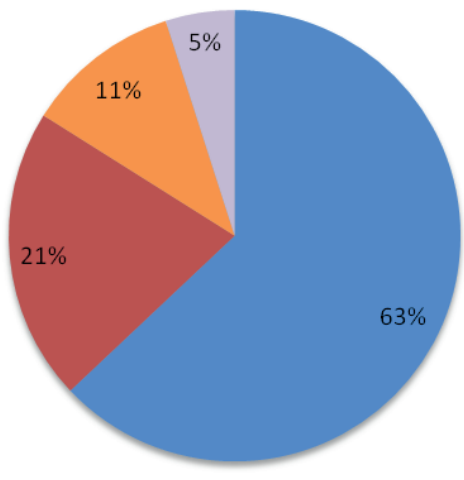

aLNB

Excision of pretreatment marked lymph node with SLNB

Excision of pretreatment marked lymph node without SLNB

nther*

* Other: $2 x$ wait and see, $1 x$ adjuvant radiotherapy, $1 x$ dependent on breast response and axillary tumour burden pre-NST.

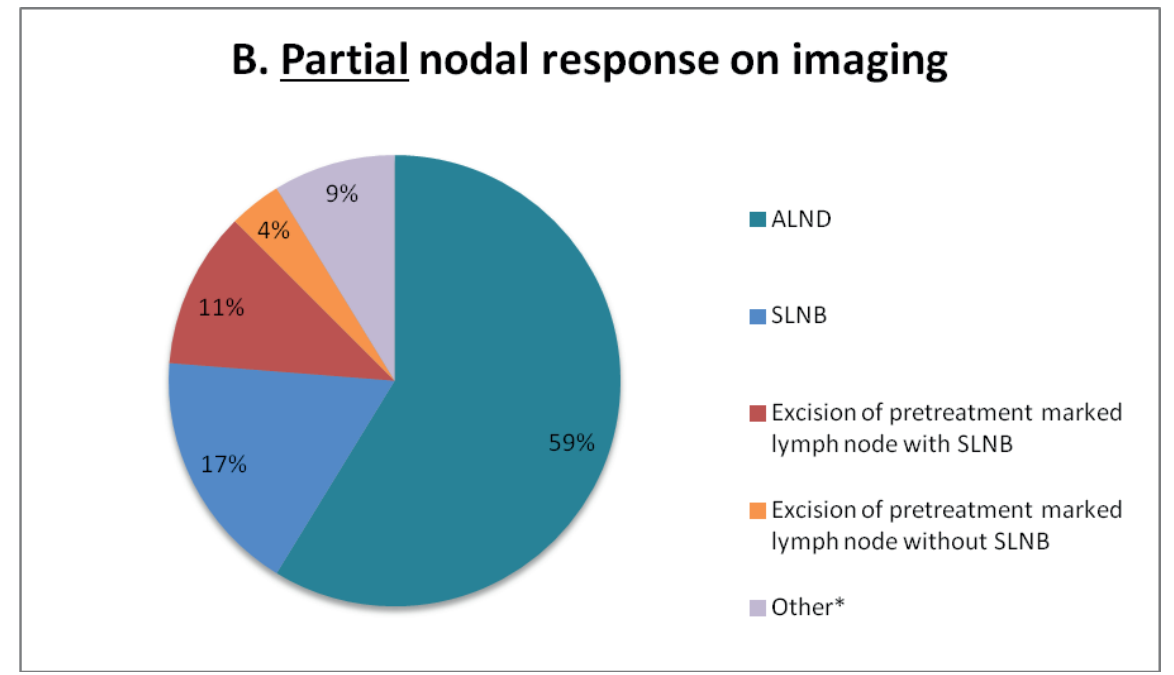

*Other: 1x ALND with axillary reverse mapping, 1x level 1 axillary dissection, 5x dependent on breast response and/or axillary tumour burden pre-NST and/or axillary tumour burden post-NST. 


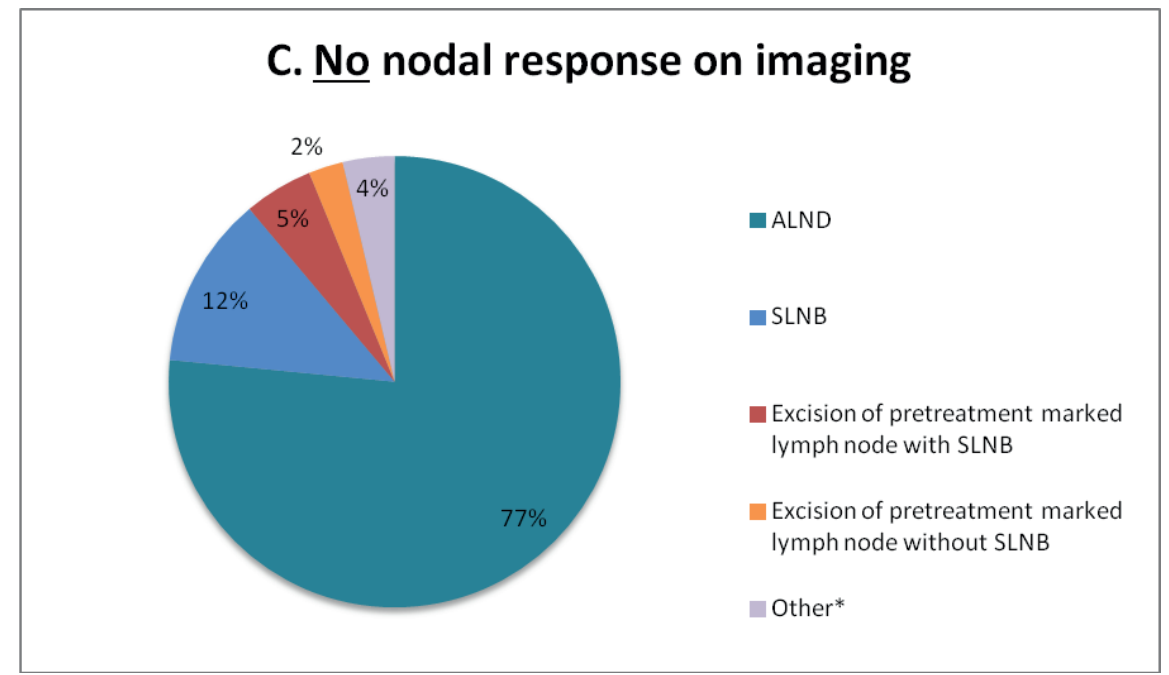

*Other: $3 x$ dependent on axillary tumour burden pre-NST and/or breast surgical treatment plan.

NB: 84 of 92 respondents who used nodal response on imaging to guide axillary restaging in $\mathrm{cN}+$ patients submitted an answer. Three respondents were excluded from this analysis because of contradicting answers

Amongst respondents that omitted ALND in $\mathrm{cN}+$ patients compared to respondents that routinely performed ALND, satisfaction rates with local protocols were $74.6 \%$ and $66 \%$ respectively $(p=.133)$ and institutional consensus rates were $60.5 \%$ and $66 \%$ respectively $(\mathrm{p}=.356)$. Patient preferences influence axillary staging and management always in $14 \%$, most of the time in $32 \%$, sometimes in $47 \%$ and never in $7 \%$ of respondents. These rates were similar for respondents that performed ALND routinely and respondents that omitted ALND in $\mathrm{cN}+$ patients.

\section{DISCUSSION}

Patients with cN+ breast cancer treated with NST achieve an axillary pCR in up to 74\%, depending on tumour characteristics. ${ }^{1,6,7}$ In pursue of omitting ALND in these patients, several less invasive staging procedures have been proposed to identify axillary PCR. This survey displays the great variety of axillary staging procedures and treatment protocols, thereby indicating a worldwide need for consensus regarding the optimal method for axillary staging after NST in $\mathrm{cN}+$ patients. 
Our survey shows that axillary staging and management vary from a minimum 'wait and see' approach (in case of complete nodal response on imaging) to a 'most extensive' ALND combined with adjuvant radiotherapy. A survey amongst members of the American society of breast surgeons indicated that only $15 \%$ still performed ALND in all $\mathrm{cN}+$ patients after NST. ${ }^{8}$ In our current survey, as much as $57.3 \%$ still performed ALND routinely in all $\mathrm{cN}+$ patients after NST. Interestingly, the survey by Caudle et al. documented that respondents that had not incorporated less invasive staging procedures were more aware of their downsides (like the false negative rates of the Z1071, Sentina and SN-FNAC trials). ${ }^{8}$ We did not explicitly ask respondents to provide reasons for still performing ALND, but several respondents voluntarily reported "concerns on long-term oncologic safety" of less invasive procedures. This indicates that there is still need for convincing evidence to support replacing ALND with less invasive procedures.

Over recent years, two methods have been proposed for axillary staging that involve excision of the pre-treatment marked positive lymph node, i.e. MARI (in which only the marked lymph node is excised after NST) ${ }^{4}$ and TAD/RISAS (in which both the marked lymph node and the sentinel lymph nodes are excised after NST). ${ }^{5,9,10}$ The marking of positive lymph nodes prior to NST, was performed by $1 / 3$ of respondents. Even respondents who routinely performed ALND, reported marking positive lymph nodes prior to NST (27\%). This was comparable to the results of the previously mentioned survey, which indicates that specialists do not always aim to retrieve the marked lymph node during surgery. Trial participation might be a reason for this. Widespread implementation of marking the positive lymph node prior to NST may be hampered by lack of resources or experience to perform this procedure.

Besides excision of the marked lymph node, the SLNB has been proposed to replace ALND. ${ }^{1,11-13}$ Although accuracy of SLNB has been questioned, our survey shows that a substantial number of respondents does opt for solitary post-NST SLNB to enable subsequent omission of ALND in $\mathrm{cN}+$ patients. Several currently accruing trials focus on different less invasive axillary management options for $\mathrm{CN}+$ patients treated with NST (amongst others RISAS ${ }^{10}$, TAXIS ${ }^{14}$, Alliance $11202^{15}$, NSABP-51/RTOG $1304^{16}$ ). While awaiting results of these ongoing trials to reach consensus and to ensure consistent axillary treatment plans, it is important to inform patients adequately on the benefits and shortcomings of the different available options. Less invasive staging procedures may avoid the morbidity associated with ALND, but may also leave therapy resistant disease behind (of which implications on prognosis are yet unknown).

The vast majority (85\%) of respondents who perform less invasive restaging of the axilla with possible omission of ALND relies on the degree of nodal response on 
imaging to guide axillary staging. Several imaging modalities have previously been studied for their accuracy regarding nodal response assessment. ${ }^{17-19}$ In general, imaging is associated with low accuracy for discriminating between axillary PCR and axillary residual disease. Therefore, its use is impeded in clinical practice. Nonetheless, some studies reported on the accuracy of SLNB when performed only in patients who have a complete nodal response on imaging and concluded that incorporating imaging may decrease the risk of missing residual disease. ${ }^{20-22}$

This survey has a few limitations. The survey was directed at all breast cancer specialists, yet over $90 \%$ of respondents comprised surgeons and surgical oncologists. Approximately $25 \%$ of respondents were practicing outside of Europe, which could further add to the wide variety of reported practices. Comments provided by respondents indicated that our survey did not cover all current axillary staging and management practices. Amongst voluntarily reported practices not covered in our survey were: considering breast response as a factor for omission of ALND, sampling lymph nodes with FNAC or CNB after NST to guide axillary surgery, performing SLNB with removing at least 4 sentinel lymph nodes and determining the need for radiotherapy prior to NST. Regarding breast response, previous studies have shown a correlation between a complete response in the breast and ypNO status. ${ }^{23,24}$ However, this link is especially strong in cNO patients. When it comes to $\mathrm{cN}+$ patients, this correlation is weak and breast PCR should therefore not be used to guide axillary surgery.

Some respondents questioned whether the development of recurrences following omission of ALND in $\mathrm{CN}+$ patients treated with NST may have legal repercussions. Although the answer options in our survey did not cover all beliefs and practices, our survey did manage to provide a thorough overview of the wide variety of current practices and the associated significant rates for lack of consensus and dissatisfaction with local protocols.

\section{Conclusion}

In conclusion, axillary staging and management practices for $\mathrm{cN}+$ patients treated with NST appear to vary widely. This indicates the need for a consensus. Breast cancer specialists should continue to join efforts to encourage $\mathrm{cN}+$ patients to participate in clinical trials or registries regarding axillary staging and management after NST. Consequently, we can gather evidence and crystallize accurate and safe axillary staging and management of $\mathrm{cN}+$ patients treated with NST. 


\section{ACKNOWLEDGMENTS}

We thank all participants for contributing to this study. We thank the following Associations for assistance with circulating the survey: Association of Breast Surgery, British Association of Surgical Oncology and European Society of Surgical Oncology. JM Simons received salary from the Dutch Cancer Society (KWF Kankerbestrijding). 


\section{REFERENCES}

1. van Nijnatten TJ, Schipper RJ, Lobbes MB, Nelemans PJ, Beets-Tan RG, Smidt ML. The diagnostic performance of sentinel lymph node biopsy in pathologically confirmed node positive breast cancer patients after neoadjuvant systemic therapy: A systematic review and meta-analysis. Eur J Surg Oncol. 2015;41(10):1278-1287.

2. Mougalian SS, Hernandez M, Lei X, et al. Ten-year outcomes of patients with breast cancer with cytologically confirmed axillary lymph node metastases and pathologic complete response after primary systemic chemotherapy. JAMA Oncol. 2016;2(4):508-516.

3. Hennessy BT, Hortobagyi GN, Rouzier R, et al. Outcome after pathologic complete eradication of cytologically proven breast cancer axillary node metastases following primary chemotherapy. J Clin Oncol. 2005;23(36):9304-9311.

4. Donker M, Straver ME, Wesseling J, et al. Marking axillary lymph nodes with radioactive iodine seeds for axillary staging after neoadjuvant systemic treatment in breast cancer patients: the MARI procedure. Ann Surg. 2015;261(2):378-382.

5. Caudle AS, Yang WT, Krishnamurthy S, et al. Improved axillary evaluation following neoadjuvant therapy for patients with node-positive breast cancer using selective evaluation of clipped nodes: Implementation of targeted axillary dissection. J Clin Oncol. 2016;34(10):1072-1078.

6. Dominici LS, Negron Gonzalez VM, Buzdar AU, et al. Cytologically proven axillary lymph node metastases are eradicated in patients receiving preoperative chemotherapy with concurrent trastuzumab for HER2-positive breast cancer. Cancer. 2010;116(12):2884-2889.

7. van la Parra RF, Kuerer HM. Selective elimination of breast cancer surgery in exceptional responders: historical perspective and current trials. Breast Cancer Res. 2016;18(1):28.

8. Caudle AS, Bedrosian I, Milton DR, et al. Use of sentinel lymph node dissection after neoadjuvant chemotherapy in patients with node-positive breast cancer at diagnosis: Practice patterns of American Society of Breast Surgeons Members. Ann Surg Oncol. 2017;24(10):2925-2934.

9. Siso C, de Torres J, Esgueva-Colmenarejo A, et al. Intraoperative ultrasound-guided excision of axillary clip in patients with node-positive breast cancer treated with neoadjuvant therapy (ILINA Trial) : A new tool to guide the excision of the clipped node after neoadjuvant treatment. Ann Surg Oncol. 2018;25(3):784-791.

10. van Nijnatten TJA, Simons JM, Smidt ML, et al. A novel less-invasive approach for axillary staging after neoadjuvant chemotherapy in patients with axillary node-positive breast cancer by combining radioactive lodine seed localization in the axilla with the sentinel node procedure (RISAS): A Dutch prospective multicenter validation study. Clin Breast Cancer. 2017;17(5):399-402.

11. Boileau JF, Poirier B, Basik M, et al. Sentinel node biopsy after neoadjuvant chemotherapy in biopsyproven node-positive breast cancer: the SN FNAC study. J Clin Oncol. 2015;33(3):258-264.

12. Boughey JC, Suman VJ, Mittendorf EA, et al. Sentinel lymph node surgery after neoadjuvant chemotherapy in patients with node-positive breast cancer: the ACOSOG Z1071 (Alliance) clinical trial. JAMA. 2013;310(14):1455-1461.

13. Kuehn T, Bauerfeind I, Fehm T, et al. Sentinel-lymph-node biopsy in patients with breast cancer before and after neoadjuvant chemotherapy (SENTINA): a prospective, multicentre cohort study. Lancet Oncol. 2013;14(7):609-618.

14. Henke G, Knauer M, Ribi K, et al. Tailored axillary surgery with or without axillary lymph node dissection followed by radiotherapy in patients with clinically node-positive breast cancer (TAXIS): study protocol for a multicenter, randomized phase-III trial. Trials. 2018;19(1):667.

15. NCT01901094. Comparison of axillary lymph node dissection with axillary radiation for patients with node-positive breast cancer treated with chemotherapy. Principal investigator: Judy Boughey. Mayo Clinic. Availableat:https://clinicaltrials.gov/ct2/show/NCT01901094. Accessed January 24, 2019.

16. NСT01872975. Standard or comprehensive radiation therapy in treating patients with early-stage breast cancer previously treated with chemo-therapy and surgery. Principal investigator: Norman Wolmar, MD. NSABPFoundation Inc. Available at: https://clinicaltrials.gov/ct2/show/NCT01872975. Accessed January 24, 2019. 
17. van Nijnatten TJA, Schipper RJ, Lobbes MBI, et al. Diagnostic performance of gadofosveset-enhanced axillary MRI for nodal (re)staging in breast cancer patients: results of a validation study. Clin Radiol. 2018;73(2):168-175.

18. Schipper RJ, Moossdorff M, Beets-Tan RGH, Smidt ML, Lobbes MBI. Noninvasive nodal restaging in clinically node positive breast cancer patients after neoadjuvant systemic therapy: a systematic review. Eur J Radiol. 2015;84(1):41-47.

19. Le-Petross HT, McCall LM, Hunt KK, et al. Axillary ultrasound identifies residual nodal disease after chemotherapy: Results from the American College of Surgeons Oncology Group Z1071 trial (Alliance). AJR Am J Roentgenol. 2018;210(3):669-676.

20. Boughey JC, Ballman KV, Hunt KK, et al. Axillary ultrasound after neoadjuvant chemotherapy and its impact on sentinel lymph node surgery: Results from the American College of Surgeons Oncology Group Z1071 trial (Alliance). J Clin Oncol. 2015;33(30):3386-3393.

21. Kang E, Chung IY, Han SA, et al. Feasibility of sentinel lymph node biopsy in breast cancer patients with initial axillary lymph node metastasis after primary systemic therapy. J Breast Cancer. 2011; 14(2):147-152.

22. Pinero-Madrona A, Escudero-Barea MJ, Fernandez-Robayna F, et al. Selective sentinel lymph node biopsy after neoadjuvant chemotherapy in breast cancer: results of the GEICAM 2005-07 study. Cir Esp. 2015;93(1):23-29.

23. Samiei S, van Nijnatten TJA, de Munck L, et al. Correlation between pathologic complete response in the breast and absence of axillary lymph node metastases after neoadjuvant systemic therapy. Ann surg. 2018.

24. Tadros AB, Yang WT, Krishnamurthy S, et al. Identification of patients with documented pathologic complete response in the breast after neoadjuvant chemotherapy for omission of axillary surgery. JAMA Surg. 2017;152(7):665-670. 




\section{PART II}

In search of an accurate less invasive axillary staging method for node positive breast cancer 



\section{CHAPTER}

Diagnostic accuracy of different surgical procedures for axillary staging after neoadjuvant systemic therapy in node-positive breast cancer: a systematic review and meta-analysis

JM Simons

TJA van Nijnatten

CC van der Pol

EJT Luiten

LB Kloppert

ML Smidt

Ann Surg. 2019;269(3):432-442 


\section{ABSTRACT}

\section{Objective}

The aim of this study was to perform a systematic review and meta-analysis to assess the accuracy of different surgical axillary staging procedures compared with ALND.

\section{Summary of background data}

Optimal axillary staging after neoadjuvant systemic therapy (NST) in node-positive breast cancer is an area of controversy. Several less invasive procedures, such as sentinel lymph node biopsy (SLNB), marking axillary lymph node with radioactive iodine seed (MARI), and targeted axillary dissection (a combination of SLNB and a MARI-like procedure), have been proposed to replace the conventional axillary lymph node dissection (ALND) with its concomitant morbidity.

\section{Methods}

PubMed and Embase were searched for studies comparing less invasive surgical axillary staging procedures to ALND to identify axillary burden after NST in patients with pathologically confirmed node-positive breast cancer ( $\mathrm{cN}+$ ). A meta-analysis was performed to compare identification rate (IFR), false-negative rate (FNR), and negative predictive value (NPV).

\section{Results}

Of 1132 records, 20 unique studies with 2217 patients were included in quantitative analysis: 17 studies on SLNB, 1 study on MARI, and 2 studies on a combination procedure. Overall axillary pathologic complete response rate was $37 \%$. For SLNB, pooled rates of IFR and FNR were $89 \%$ and $17 \%$. NPV ranged from $57 \%$ to $86 \%$. For MARI, IFR was $97 \%$, FNR $7 \%$, and NPV $83 \%$. For the combination procedure, IFR was $100 \%$, FNR ranged from $2 \%$ to $4 \%$, and NPV from $92 \%$ to $97 \%$.

\section{Conclusion}

Axillary staging by a combination procedure consisting of SLNB with excision of a preNST marked positive lymph node appears to be most accurate for axillary staging after NST. More evidence from prospective multicenter trials is needed to confirm this. 


\section{INTRODUCTION}

De-escalation of axillary surgery in clinically node positive $(\mathrm{cN}+)$ breast cancer patients is a topic of debate. The significant number of patients with axillary pathologic complete response (ax-pCR) resulting from increased use of neoadjuvant systemic therapy (NST) urges the need for a less invasive procedure to replace the conventional axillary lymph node dissection (ALND) in order to diminish unnecessary morbidity in patients with ax-pCR. ${ }^{1-3}$ Seeking for a less invasive procedure, both prevention of unnecessary morbidity and preservation of oncologic safety are of utmost importance.

Until recently, ALND was routinely performed after NST in cN+ patients, irrespective of axillary response to NST. Recent surveys among members of the American Society of Breast Surgeons as well as Dutch surgeons reported changes in axillary surgery in $\mathrm{CN}+$ patients treated with NST. ${ }^{4,5}$ The majority of specialists were willing to replace ALND by a less invasive staging procedure in patients with a favorable treatment response. A wide variety of less invasive staging procedures such as sentinel lymph node biopsy (SLNB), removal of a marked pathologically-proven positive lymph node (MARI and MARI-like procedures), or a combination of these two procedures (eg, targeted axillary dissection) are incorporated in clinical practice according to local preferences. ${ }^{6-9}$ These results denote the lack of consensus on the preferred procedure for axillary staging after NST in pretreatment $\mathrm{cN}+$ patients. Up to now, it is not clear which procedure is optimal.

The aim of this review is to provide an overview of different less invasive procedures for axillary staging after NST in pretreatment $\mathrm{cN}+$ patients, which are currently in use. By evaluating the accuracy of different less invasive axillary staging procedures, we aim to determine the optimal procedure for axillary staging after NST in cN+ breast cancer to safely replace ALND.

\section{METHODS}

\section{Criteria for considering studies for this review}

This systematic review was performed according to the Preferred Reporting Items for Systematic Reviews and Meta-Analyses (PRISMA) guidelines for diagnostic test accuracy. ${ }^{10}$ A systematic literature search was performed by the first author (JM) for randomized controlled trials, cohort studies, and case-control studies testing less invasive axillary staging procedures after NST in $\mathrm{cN}+$ breast cancer patients treated with NST. Studies were only included if nodal positivity was pathologically confirmed 
before starting with NST. Any study in which a less invasive axillary staging procedure was compared with the gold standard, that is, ALND, was included. In case completion ALND (CALND) was not performed routinely, studies were not considered for quantitative analysis. If relevant studies included both $\mathrm{cNO}$ and $\mathrm{cN}+$ patients, only the $\mathrm{cN}+$ patients were considered for analysis. In case only part of the study population consisted of patients with pathologic confirmed nodal positivity, only the pathologically confirmed $\mathrm{cN}+$ patients were considered for analysis. When it was not possible to discriminate between $\mathrm{cNO}$ and $\mathrm{cN}+$ patients or between pathologically confirmed and non-pathologically confirmed cN+ patients, studies were excluded. Reviews, case reports, conference abstracts, and editorials were excluded. In case of inclusion of the same study population in 2 or more papers, the most extensive paper was included. Studies reporting small study populations (10 patients or less) and studies in which nodal positivity was confirmed by SLNB before NST were excluded. The primary outcome was the overall ax-pCR rate and the accuracy of the studied less invasive axillary staging procedure. Studies were therefore excluded if reported data did not allow construction of a $2 \times 2$ contingency table with absolute numbers of true positive (TP), true negative (TN), false-positive (FP), and false-negative (FN) test results. FP is always 0 , as the index test and reference test are considered the same in case of a positive index test result (ie, presence of residual axillary disease). The secondary outcome was the identification rate (IFR) of the studied less invasive axillary staging procedure.

\section{Search methods for identification of studies}

The following electronic databases were searched until April 20, 2018, with no restriction on language or date of publication: Medline (via PubMed) and EMBASE (via EMBASE.com). A health sciences librarian was consulted to help develop a detailed search strategy. Details of the full search strategies in both databases are provided in Appendix 4.1. The reference lists of included studies and existing reviews were manually checked for additional relevant studies.

\section{Selection of studies and quality assessment}

Duplicate references were identified and removed with Endnote. Titles and abstracts of all remaining references were scanned independently by 2 authors (JS and TVN). Subsequently, these 2 authors independently assessed the full text papers of all potentially eligible studies. Disagreement was resolved by mutual consensus. Eligible studies were assessed for quality using the Quality Assessment of Diagnostic Accuracy Studies 2 (QUADAS- 2) questionnaire. ${ }^{11}$ The QUADAS-2 was tailored to our analysis, as described in the guideline. Finally, all included studies were evaluated for quality by the 2 independent authors. 


\section{Data extraction and analysis}

For each included study, the following parameters were extracted: first author, year of publication, type of hospital, study design, sample size, characteristics of trial participants (including primary tumor type, TNM-stage, type of evaluation of axillary involvement, and NST regimens), type of less invasive axillary staging procedure after NST and characteristics of the procedure, type of pathological assessment of lymph nodes (including use of immunohistochemistry (IHC), definition of ax-pCR, ax-pCR rate, accuracy, and IFR of the less invasive axillary staging procedure. For each less invasive axillary staging procedure, the ax-pCR rate, IFR, false-negative rate (FNR), and negative predictive value (NPV) were calculated. Rate of ax-pCR was based on data of the contingency tables. The IFR was defined as the number of successful procedures divided by the total number of patients in whom the procedure was attempted. The FNR was defined as the number of FN divided by the total number of patients with presence of residual axillary disease [FN / (FN + TP)]. The NPV was defined as the number of TN divided by the total number of patients with a negative test result [TN / $(\mathrm{TN}+\mathrm{FN})]$. As FP cannot occur, numbers of TP, TN, and FN were documented for each procedure and FP was always documented to be 0 (in case FP was reported to be $>0$ in the record, this number was added up to the TP number). Statistical analysis was performed with Stata/SE Statistical Software for Windows, version 14.2 (StataCorp LP, College Station, TX). To calculate pooled proportions for ax-pCR rate, IFR and FNR random-effects models for meta-analysis were used with $95 \%$ exact confidence intervals (Cls) with help of the metaprop command. ${ }^{12}$ All considered outcomes are presented in forest plots including pooled estimates. Chi-squared test was performed to test for statistical heterogeneity and was quantified by $I^{2}$-index. ${ }^{13}$ As recommended in the Cochrane Handbook for Diagnostic Test Accuracy Reviews, ${ }^{14}$ reporting bias (eg, publication bias) was not assessed.

Subgroup analyses were performed to evaluate the impact of several factors on FNR. Factors that were considered relevant were number of examined lymph nodes, sampling method for SLNB, ycN status, definition of ax-pCR, and use of IHC in addition to standard H\&E evaluation. Statistical significance was considered as $p$-values (2-sided) $\leq 0.05$.

\section{RESULTS}

\section{Study selection}

In total, 1920 records were identified through database searching and reference checking. After deduplication, 1132 records were screened, which resulted in the selection of 116 records for retrieval of full texts. Assessment of full text for eligibility yielded 27 records for 
qualitative synthesis; a total of 20 records were included in quantitative synthesis. See Figure 4.1 for a flow chart depicting the study selection process.

Figure 4.1 Flow diagram depicting the study selection process.
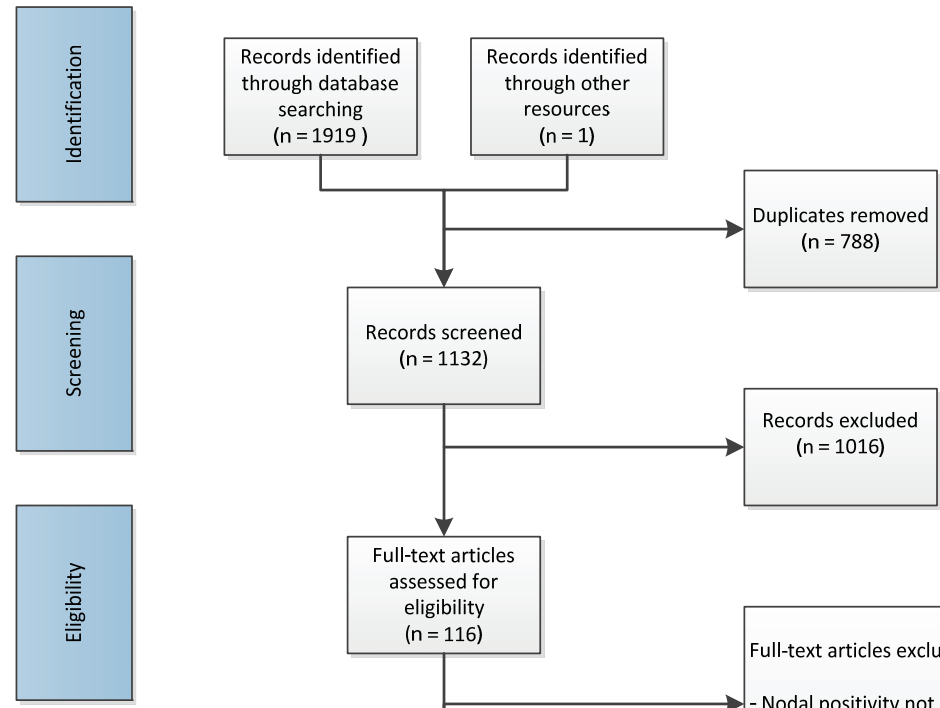

Full-text articles

assessed for

eligibility

$(n=116)$

Full-text articles excluded $(n=89)$
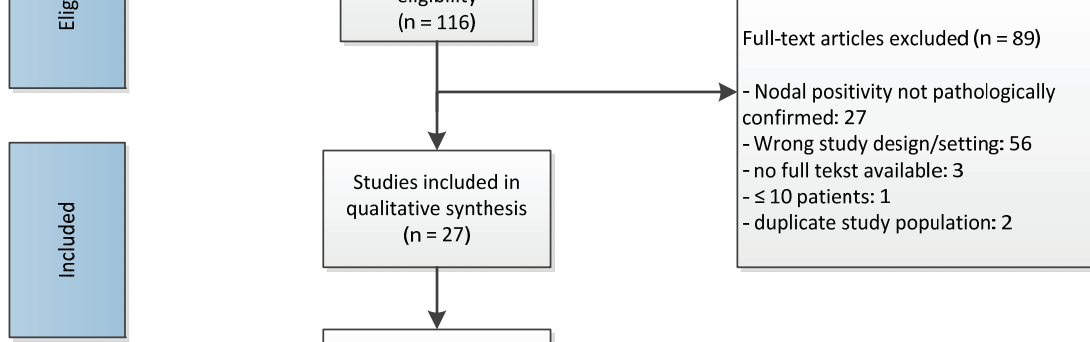

duplicate study population: 2

\section{Study characteristics}

\section{Index tests}

Three different axillary staging procedures were identified: SLNB, excision of a pretreatment marked biopsy-proven positive lymph node (hereinafter all such procedures are referred to as $\mathrm{ML}$ ), and a combination procedure involving both SLNB and ML. A total of 2217 patients were included ( 2002 for SLNB, 95 for ML, 120 for the combination procedure) in whom the axillary staging procedure was successful and followed by cALND. See Table 4.1 for general characteristics of all studies included for qualitative analysis. 


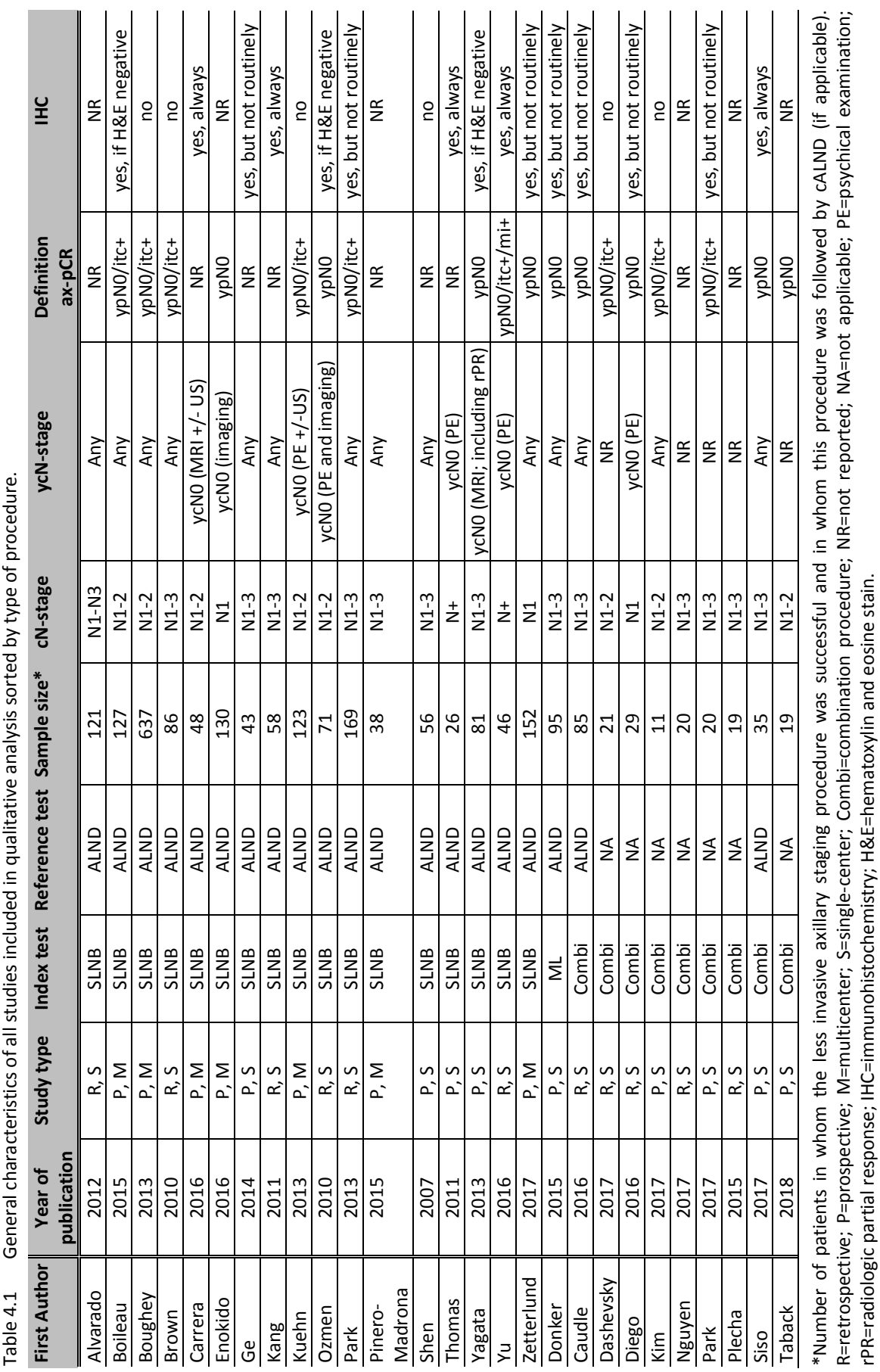




\section{Reference tests}

In 20 studies, the axillary staging procedure was always followed by ALND as part of trial protocol. A total of 17 trials investigated accuracy of SLNB, ${ }^{15-31} 1$ trial investigated $\mathrm{ML}^{8}$ and 2 trials investigated a combination procedure. ${ }^{6,32}$ Studies validating the combination procedure were scarce, yet several studies did report on cohorts of patients in whom a combination procedure was performed without routine cALND. Therefore, we decided to include these studies, 7 in total, in the qualitative analysis. ${ }^{7,33-38}$ See Table 4.2 for detailed characteristics of these studies. In this table, we have included results of a study by our own research group (manuscript submitted). As cALND was not routinely performed in these studies, they were excluded from quantitative analysis.

\section{Risk of bias and applicability}

Figure 4.2 shows the methodological quality of all included studies. In general, studies included in the quantitative analysis showed a lower risk of bias than studies included only in the qualitative analysis.

\section{Results of individual studies included in quantitative analysis}

\section{Pooled prevalence of $a x-p C R$}

The overall prevalence of ax-pCR in all 20 included studies was 37\% (see Appendix 4.2). The $I^{2}$-statistic was $57.08 \%(p<0.01)$. Test for heterogeneity between subgroups based on staging procedure was not significant, supporting the pooling of all studies in 1 overall rate.

\section{SLNB}

The IFR of SLNB was available for 16 of 17 studies. The overall IFR was $89 \%$ in a pooled sample of 2154 patients (see Appendix 4.3). For all studies, data to calculate FNR and NPV were available for a total of 2002 patients. An overall FNR of $17 \%$ was found (see Figure 4.3 ) and NPV ranged from $57 \%$ to $86 \%$ (see Table 4.3 SLNB). The $I^{2}$ statistic revealed values of variation due to heterogeneity of $68.3 \%$ for IFR and $38.7 \%$ for FNR ( $p<0.01$ and $p=0.05$, respectively). Ten studies documented the definition of ax-pCR: overall FNR was $16 \%$ when ax-pCR was defined as ypNO and $17 \%$ when ax-PCR was defined as ypNo/itc+ ( $p=0.61,1$ study defined ax-pCR as ypNo/itc+/mi+). FNR was reported for single versus dual-tracer sampling separately in 5 studies and another 6 studies used either single-tracer or dual-tracer sampling in $100 \%$ of patients. Pooled FNR was $13 \%$ for dual-tracer sampling and $16 \%$ for single-tracer sampling $(p=0.53)$. 


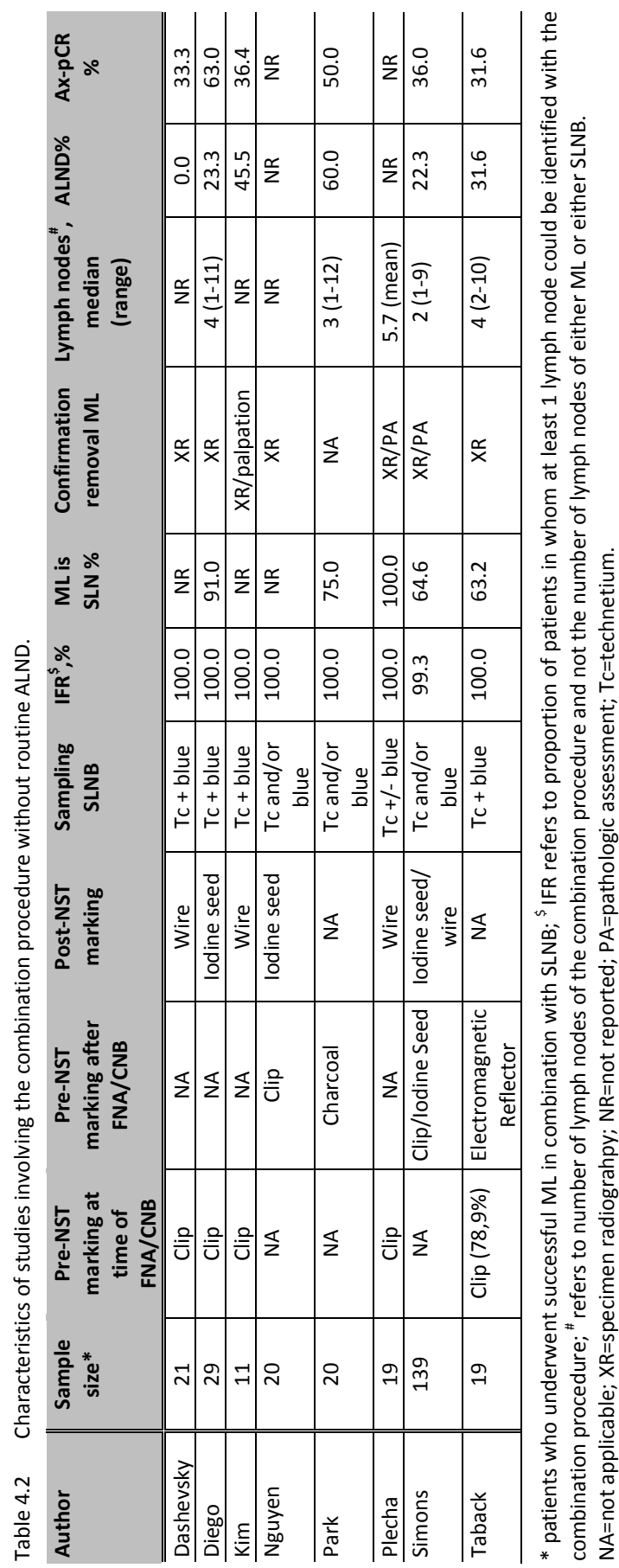


A total of 14 studies reported on the use of IHC: overall FNR was $15 \%$ when IHC was used (either always or in selected patients) versus $17 \%$ when IHC was not used $(p=0.47)$. In 6 studies, FNR was reported separately in relation to the number of SLN(s): all 6 studies reported FNR for excision of 3 or more SLNs (NB: in 1 study, this was 2 or more SLNs) and 5 of 6 studies also reported FNR for excision of <3 SLNs. Overall FNR was $8 \%$ with removal of at least 3 SLNs and $22 \%$ with removal of $<3$ SLN(s) $(p<0.0001)$. In 7 studies, only $\mathrm{cN}+$ patients with ycNO status were included. In addition to these studies, another 4 studies reported on FNR separately for patients with any ycN status versus ycNO status. Overall FNR was $14 \%$ when only patients with ycNO status were taken into account versus $18 \%$ when patients irrespective of ycN status were taken into account $(p=0.14)$.

Figure 4.2(A-B) Assessment of risk of bias.

4.2A. Studies included in quantitative analysis.

\begin{tabular}{|c|c|c|c|c|c|c|c|}
\hline \multirow[t]{4}{*}{ Study } & \multicolumn{4}{|c|}{ RISK OF BIAS } & \multicolumn{3}{|c|}{ APPLICABILITY CONCERNS } \\
\hline & PATIENT & INDEX & REFERENCE & FLOW & PATIENT & INDEX TEST & REFERENCE \\
\hline & SELECTION & TEST & STANDARD & AND & SELECTION & & STANDARD \\
\hline & & & & TIMING & & & \\
\hline Alvarado & $?$ & ? & (;) & $?$ & (:) & (:) & (;) \\
\hline Boileau & (:) & ;) & ;) & ;) & ;) & (:) & (:) \\
\hline Boughey & (;) & ;) & ;:) & ;:) & ;) & ;) & ;:) \\
\hline Brown & ? & ;) & (:) & ;:) & ? & (:) & ;:) \\
\hline Carrera & ;) & ;) & ;) & (:) & ;) & (;) & (:) \\
\hline Enokido & (:) & ;) & (:) & ;) & ;) & (-) & (:) \\
\hline $\mathrm{Ge}$ & ? & ? & ;) & (;) & ? & (:) & (:) \\
\hline Kang & : : & ? & ;:) & $?$ & ? & ? & ;:) \\
\hline Kuehn & ;) & ;) & (:) & ;) & ;) & ;) & (:) \\
\hline Ozmen & $?$ & ;) & (;) & $?$ & ;) & (:) & (:) \\
\hline Park & ? & (:) & (:) & $?$ & $?$ & ;) & (:) \\
\hline Pinero- & (:) & ? & (:) & ;) & (:) & (;) & (;) \\
\hline \multicolumn{8}{|l|}{ Madrona } \\
\hline Shen & ;) & ? & ;) & (:) & ;) & ;) & (:) \\
\hline Thomas & (;) & ? & ;) & (:) & (:) & (;) & (:) \\
\hline Yagata & (;) & (:) & (:) & ;) & ;) & ;) & (-) \\
\hline $\mathrm{Yu}$ & ? & ;:) & ;:) & ;:) & ? & (:) & (:) \\
\hline Zetterlund & (:) & (:) & (:) & (:) & (:) & (:) & (:) \\
\hline Donker & ;) & ;) & ;:) & ;) & ;) & ;) & ;) \\
\hline Caudle & :) & ;) & (:) & ? & ? & ;) & (:) \\
\hline Siso & ? & (:) & (:) & $?$ & ? & (;) & (:) \\
\hline
\end{tabular}

(:)Low Risk :High Risk ? Unclear Risk 
4.2B. Studies included in qualitative analysis only.

\begin{tabular}{lccccccc}
\hline Study & \multicolumn{3}{c}{ RISK OF BIAS } & \multicolumn{2}{c}{ APPLICABILITY CONCERNS } \\
\cline { 2 - 7 } & $\begin{array}{c}\text { PATIENT } \\
\text { SELECTION }\end{array}$ & $\begin{array}{c}\text { INDEX } \\
\text { TEST }\end{array}$ & $\begin{array}{c}\text { REFERENCE } \\
\text { STANDARD }\end{array}$ & $\begin{array}{c}\text { FLOW } \\
\text { AND } \\
\text { TIMING }\end{array}$ & $\begin{array}{c}\text { PATIENT } \\
\text { SELECTION }\end{array}$ & INDEX TEST & REFERENCE \\
STANDARD
\end{tabular}

(i)Low Risk :High Risk ? Unclear Risk

Figure 4.3 Forest plot for the FNR of SLNB.

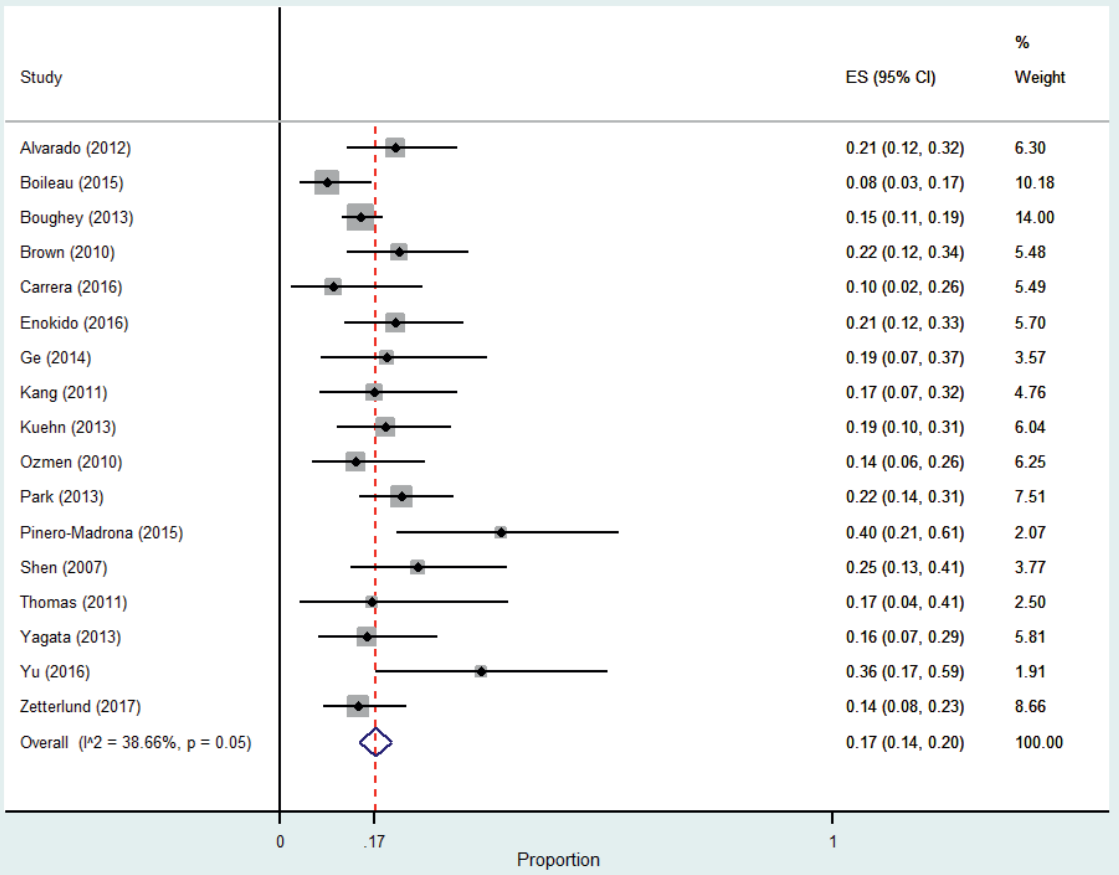

ES: effect size. The pooled FNR of SLNB is 17\% (14-20\%). 


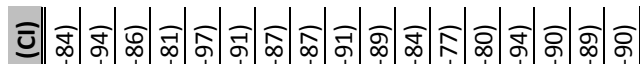

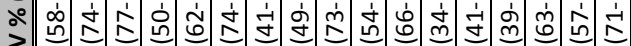

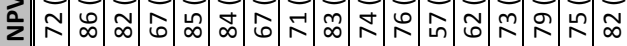

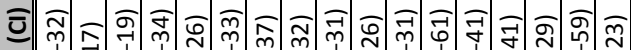

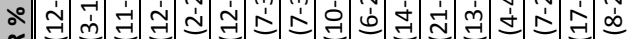

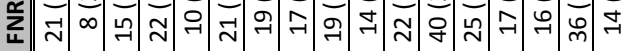

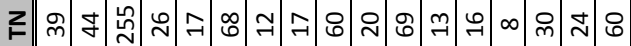

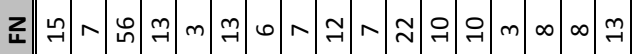

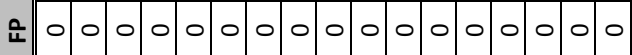

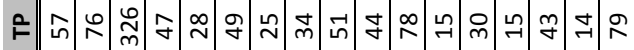
:̊n

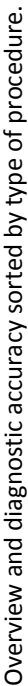

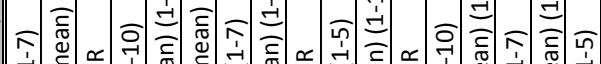
हn:

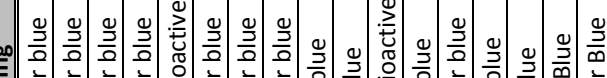

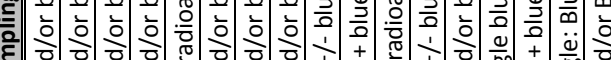
竕

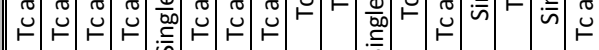

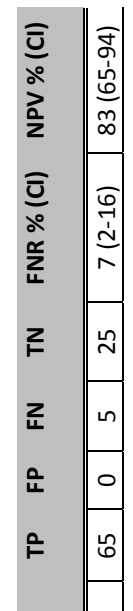

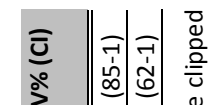

วิ

ธิ

产 仓े 仓े

3 约

$3 \quad D \quad$ 年

落

은 000 苞

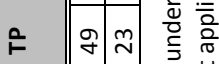

×

3

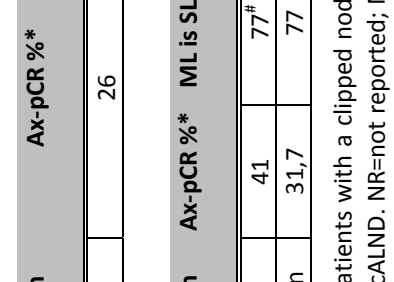

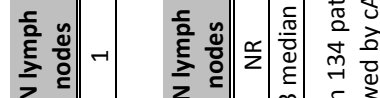

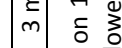

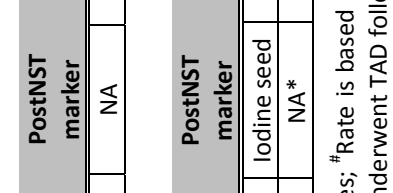

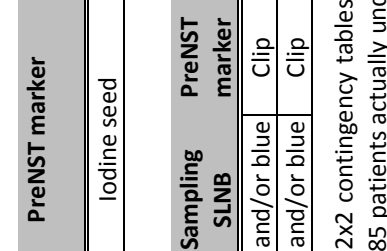

$\therefore$ ก.

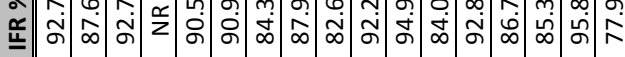

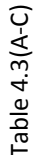

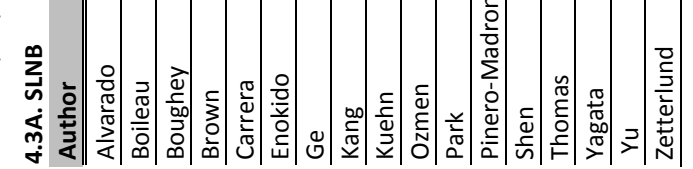

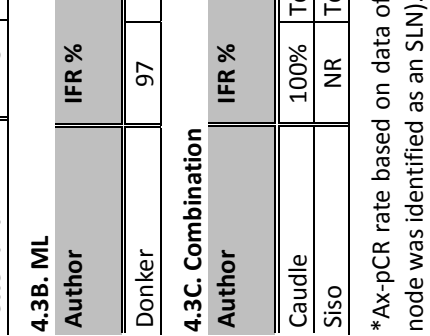


$M L$

One study reported on a ML procedure: this study involved the validation of the MARI procedure (marking axillary lymph nodes with radioactive iodine seeds) in 95 patients. In this study, the pathologically proven positive lymph node was marked with an I-125 seed pre-NST. After completion of NST, at the time of surgery, the lymph node with the lodine seedwas removed. The IFR was 97\%, the FNR 7\%, and the NPV $83.3 \%$. See Table $4.3(\mathrm{ML})$ for characteristics of this procedure.

\section{Combination procedure}

Two studies investigated a combination procedure: one involved clipping of the positive lymph node pre-NST later followed by I-125 seed localization of the clipped node post-NST in combination with SLNB and one involved clipping of the positive lymph node pre-NST followed by US-guided excision of the clipped-node in combination with SLNB. Table 4.3 (Combination) shows values for FNR and NPV for the 2 combination procedures. As only 2 studies were available for analysis, pooling of proportions was not performed. The studies appeared clinically similar, as they targeted the same population in terms of inclusion criteria, definition of ax-pCR, and use of IHC.

\section{DISCUSSION}

In this systematic review, the accuracy of 3 different procedures for axillary staging after NST in $\mathrm{cN}+$ patients was evaluated. This is the first review up to now that compared all these different less invasive staging procedures with the gold standard ALND. The goal was to provide an overview of currently available procedures in order to guide decision making regarding replacing ALND in selected $\mathrm{cN}+$ patients.

The SLNB for axillary staging after NST in $\mathrm{CN}+$ patients has been extensively studied over the past years. The SLNB procedure is widely accepted as axillary staging procedure in cNO patients. Even when performed after NST, the accuracy of SLNB for cNO patients is accepted. ${ }^{39}$ SLNB for axillary staging after NST in pre-treatment cN+ patients, however, is associated with unacceptably high rates of FNR. In 2015, the accuracy of SLNB in cN+ patients after NST was evaluated in a systematic review that included 8 studies with pathologically proven $\mathrm{cN}+$ patients. ${ }^{40}$ That review reported an overall FNR of $15 \%$ and the NPV of SLNB did not exceed $86 \% .^{40}$ In the current metaanalysis, a total of 17 studies with 2002 patients (the 8 studies of the previous review were also included) were analyzed. The overall FNR is $17 \%$ and the NPV still does not exceed 86\%: in case SLNB predicts ax-PCR, residual axillary disease is actually missed in at least 1 in 6 patients. The overall IFR is $89 \%$. Previous studies reported multiple 
factors that may improve IFR and accuracy of SLNB, for example, using dual-tracer sampling technique, evaluating the SLNs with IHC in addition to standard H\&E evaluation and removing 3 or more SLNs. As IHC and single- versus dual-tracer sampling was not used consistently within and between studies, it is not possible to draw definite conclusions from this review on whether/or not specific sampling and pathologic evaluation methods should be promoted. Our results did show that FNR was favorable (yet not statistically significant) for both dual-tracer sampling and pathologic evaluation with IHC. FNR was also favorable for patients with ycNO status based on physical examination and/or imaging compared with any ycN status (FNR of $14 \%$ vs. $18 \%, p=0.14$ ). Regarding the number of SLNs, removing $\geq 3$ lymph nodes was associated with a significantly better FNR in our meta-analysis ( $8 \%$ vs. $22 \%, p<0.0001$ ). However, removing $\geq 3$ SLNs is not achievable in a significant number of patients ${ }^{17}$ and whether this will be achieved is unpredictable preoperatively. This renders SLNB impractical, as random node-picking should be discouraged. Currently recruiting studies as Alliance 11202 and NSABP-51/RTOG 1304 will determine whether the SLNB, despite its rather poor overall accuracy and shortcomings, can have a place in axillary staging after NST in $\mathrm{cN}+$ patients. ${ }^{41,42}$

The MARI procedure was the first ML procedure to be proposed as an alternative to SLNB for axillary staging after NST in $\mathrm{CN}+$ patients. ${ }^{8}$ By marking the pathologically proven positive lymph node before start of NST, it was expected to enable accurate assessment of treatment response after completion of NST. The MARI procedure was validated in 1 single-center trial with 95 patients. This study reported an improved FNR (7\%), but the NPV of $83.3 \%$ was less favorable, that is, in 1 of 6 patients with a negative MARI, axillary residual disease is left behind. Therefore, equally to SLNB, MARI as a stand-alone procedure is insufficiently accurate to safely replace ALND. Despite the shortcomings of MARI (potential of missing residual axillary disease and limited evidence by 1 single-dedicated breast cancer-center trial to support this procedure), it is already implemented in clinical practice. A recent publication suggests combining information on axillary burden on pre-NST PET-CT (ie, number of suspicious lymph nodes: $<4$ vs. $\geq 4$ ) with MARI outcome ${ }^{9}$ to determine adjuvant axillary treatment: no further axillary treatment, axillary radiotherapy, or cALND with axillary radiotherapy. Results of 1 prospective implementation study showed that this treatment strategy indeed results in a major reduction of ALND. ${ }^{43}$ Data of longer follow-up have to determine whether implementation of this protocol is untimely and whether it does not only reduce morbidity but also preserves oncologic safety in terms of disease-free and overall survival. Prospective trials with sufficient follow-up are therefore urgently needed.

In the Z1071 trial, a clip was placed in the positive lymph node before NST in a subset of patients. ${ }^{44}$ The trial protocol did not require surgeons to selectively target and 
remove the clipped node at time of surgery, but did encourage surgeons and pathologists to document whether the clipped node was located in the SLNB or ALND specimen. In 141 of 170 patients with a clipped node, the location of the clipped node was documented: $75.9 \%$ in the SLNB specimen and $24.1 \%$ in the ALND specimen. This suggested that removing the clipped node together with $\operatorname{SLN}(\mathrm{s})$ at time of surgery may improve accuracy of SLNB and may possibly overcome shortcomings associated with SLNB or MARI if used as stand-alone procedures. Up to now, only 2 trials evaluated accuracy of such a combination procedure and were included in our meta-analysis. ${ }^{6,32}$ This procedure is associated with excellent IFRs. Caudle et $\mathrm{al}^{6}$ confirmed that the clipped node does not necessarily have to be a SLN, as this was the case in only $77 \%$. Furthermore, FNR is low ( $2 \%$ to $4 \%$ ) and NPV is high (92\% to $97 \%$ ). These results are promising: when ax-pCR is predicted, residual axillary disease is missed in 1 in 12 to 33 patients. The evidence for this procedure is yet limited with only 2 trials available ( 1 retrospective and 1 prospective study), involving small sample sizes and single-center study designs. The ongoing Dutch RISAS trial (NCT02800317 at https://clinicaltrials.gov) will prove whether the promising results of a combination procedure can be confirmed in a large, prospective, multicenter trial. ${ }^{45}$

Although evidence to support replacing ALND by less invasive procedures is limited, several reports have been recently published on implementation of such procedures, especially procedures involving excision of the ML and SLNs. A variety of methods are used to target the pathologically proven positive lymph node: marking with a clip preNST followed by placing an iodine seed or wire in the clipped node post-NST $7,33-35,37$ and primary marking with an iodine seed, clip, charcoal, or electromagnetic reflector. $^{36,38}$ Also, the time of marking the lymph node pre-NST differs: either immediately at time of $\mathrm{FNAC/CNB},{ }^{7,33,34,37}$ at a second appointment once metastatic burden of the punctured lymph node is confirmed by the pathologist ${ }^{35,36}$ or even at both occasions. ${ }^{38}$ Currently, further research has to define which combination procedure is most accurate, patient-friendly, and cost-effective. Identification of the $\mathrm{ML}$ at time of surgery is highly feasible, provided that clipping (with/without secondary localization of the clip) of the node was successful. Success rates of this part of the procedure are often not sufficiently reported and may be improved to further optimize combination procedures.

The abovementioned 3 different staging procedures intend to offer a less invasive strategy compared with the conventional ALND, yet $\geq 10$ lymph nodes are removed in some patients with SLNB and combination procedures. It is important to realize that these procedures serve as a staging procedure to identify ax-pCR and not as a managing procedure to remove all residual diseases. Hence, it should be the primary goal to remove as few lymph nodes as possible. In this way, patients with ax-pCR can truly benefit from less invasive staging procedures. At the same time, when these 
procedures identify axillary residual disease, adjuvant axillary treatment plans should consist of cALND. Results of the Alliance 11202 and NSABP-51/RTOG 1304 trials have to be awaited to determine whether CALND may be replaced by axillary radiotherapy. ${ }^{41,42}$

As this review is limited by the heterogeneity of included studies, results of the review should be interpreted with caution. The random effects model that was used for statistical analysis takes in account that, although similar interventions were studied, different populations were included. Factors such as definition of ax-PCR, sampling method for SLNB, and use of IHC for pathologic assessment of lymph nodes may all impact accuracy of the studied intervention. These factors differed widely among included studies and further research is necessary to determine, among others, what should be the preferred definition of ax-pCR. The prognostic impact of residual ITCs and micrometastasis may be different for patients treated in the neoadjuvant compared with adjuvant setting, as they might be therapy-resistant. A retrospective study of $\mathrm{cN}+$ patients treated with NAC and always followed by ALND suggested that patients with residual ITCs and micrometastases carry a similar prognosis as patients with ypN0. ${ }^{46}$ These results have yet to be confirmed in trials where patients with ypNO and ITCS or micrometastasis did not undergo ALND. In addition, the value of IHC has not yet been thoroughly studied, as most studies that used IHC in addition to standard H\&E evaluation, did so randomly, and not in a routine matter. Contrary to improving accuracy of detecting residual axillary disease, a potential undesired result of IHC may be detection of residual disease that would have otherwise been left undetected (of which implications on prognosis and need for adjuvant treatment are unknown). The question whether IHC may not only result in improved accuracy but may also result in overtreatment is yet left unanswered.

In this review, we only included patients in whom cN+ status was pathologically proven before NST. This is particularly important to determine true accuracy of the different staging procedures. When patients who are expected to be $\mathrm{cN}+$ based on physical examination or imaging only, the number of true negatives rises and the chance to have false negatives decreases. This may result in a false impression of improved rates of FNR and NPV.

In conclusion, the SLNB as well as ML procedures seem insufficiently accurate as stand-alone procedures for axillary staging after NST in $\mathrm{cN}+$ patients. Accuracy of these procedures may improve by taken in account axillary burden on pre-NST and/or post-NST imaging. A combination procedure involving excision of the ML and SLNs appears most accurate for axillary staging and has the lowest risk of missing axillary residual disease when ax-pCR is predicted. More evidence from prospective multicenter trials is needed to confirm this. 


\section{ACKNOWLEDGMENTS}

We thank the following individuals for assistance with search strategies and statistical analysis:

P.H. Wiersma and F.P. Weijdema, health science librarians, Utrecht University Library.

J.B. Reitsma, clinical epidemiologist, Julius Center for Health Sciences and Primary Care, University Medical Center Utrecht.

R.J.P.M. Scholten, professor of clinical epidemiology, Julius Center for Health Sciences and Primary Care, University Medical Center Utrecht. 


\section{REFERENCES}

1. van Bommel AC, Spronk PE, Vrancken Peeters MT, et al. Clinical auditing as an instrument for quality improvement in breast cancer care in the Netherlands: The national NABON Breast Cancer Audit. J Surg Oncol. 2017;115(3):243-249.

2. Vugts G, Maaskant-Braat AJ, Nieuwenhuijzen GA, Roumen RM, Luiten EJ, Voogd AC. Patterns of care in the administration of neo-adjuvant chemotherapy for breast cancer. A population-based study. Breast J. 2016;22(3):316-321.

3. DICA. Annual report of the NABON Breast Cancer Audit 2016 - Dutch Institute for Clinical Auditing. https://dica.nl/jaarrapportage-2016/nbca.

4. Caudle AS, Bedrosian I, Milton DR, et al. Use of sentinel lymph node dissection after neoadjuvant chemotherapy in patients with node-positive breast cancer at diagnosis: Practice patterns of American Society of Breast Surgeons Members. Ann Surg Oncol. 2017;24(10):2925-2934.

5. Vugts G, Maaskant-Braat AJ, de Roos WK, Voogd AC, Nieuwenhuijzen GA. Management of the axilla after neoadjuvant chemotherapy for clinically node positive breast cancer: A nationwide survey study in The Netherlands. Eur J Surg Oncol. 2016;42(7):956-964.

6. Caudle AS, Yang WT, Krishnamurthy S, et al. Improved axillary evaluation following neoadjuvant therapy for patients with node-positive breast cancer using selective evaluation of clipped nodes: Implementation of targeted axillary dissection. J Clin Oncol. 2016;34(10):1072-1078.

7. Diego EJ, McAuliffe PF, Soran A, et al. Axillary staging after neoadjuvant chemotherapy for breast cancer: A pilot study combining sentinel lymph node biopsy with radioactive seed localization of pretreatment positive axillary lymph nodes. Ann Surg Oncol. 2016;23(5):1549-1553.

8. Donker M, Straver ME, Wesseling J, et al. Marking axillary lymph nodes with radioactive iodine seeds for axillary staging after neoadjuvant systemic treatment in breast cancer patients: the MARI procedure. Ann Surg. 2015;261(2):378-382.

9. Koolen BB, Donker M, Straver ME, et al. Combined PET-CT and axillary lymph node marking with radioactive iodine seeds (MARI procedure) for tailored axillary treatment in node-positive breast cancer after neoadjuvant therapy. Br J Surg. 2017;104(9):1188-1196.

10. Mclnnes MDF, Moher D, Thombs BD, et al. Preferred reporting items for a systematic review and meta-analysis of diagnostic test accuracy studies: the PRISMA-DTA statement. JAMA. 2018;319(4):388-396.

11. Whiting PF, Rutjes AW, Westwood ME, et al. QUADAS-2: a revised tool for the quality assessment of diagnostic accuracy studies. Ann Intern Med. 2011;155(8):529-536.

12. Nyaga VN, Arbyn M, Aerts M. Metaprop: a Stata command to perform meta-analysis of binomial data. Arch Public Health. 2014;72(1):39.

13. Higgins JP, Thompson SG. Quantifying heterogeneity in a meta-analysis. Stat Med. 2002;21(11):15391558.

14. Macaskill P, Gatsonis C, Deeks JJ, Harbord RM, Takwoingi Y. Cochrane Handbook for Systematic Reviews of Diagnostic Test Accuracy. In: Deeks JJ, Bossuyt PM, Gatsonis C editor(s). Version 1.0. Vol. Chapter 10: Analysing and Presenting Results, The Cochrane Collaboration, 2010.

15. Alvarado $\mathrm{R}$, Yi $\mathrm{M}$, Le-Petross $\mathrm{H}$, et al. The role for sentinel lymph node dissection after neoadjuvant chemotherapy in patients who present with node-positive breast cancer. Ann Surg Oncol. 2012; 19(10):3177-3184.

16. Boileau JF, Poirier B, Basik M, et al. Sentinel node biopsy after neoadjuvant chemotherapy in biopsyproven node-positive breast cancer: the SN FNAC study. J Clin Oncol. 2015;33(3):258-264.

17. Boughey JC, Suman VJ, Mittendorf EA, et al. Sentinel lymph node surgery after neoadjuvant chemotherapy in patients with node-positive breast cancer: the ACOSOG Z1071 (Alliance) clinical trial. JAMA. 2013;310(14):1455-1461.

18. Brown AS, Hunt KK, Shen J, et al. Histologic changes associated with false-negative sentinel lymph nodes after preoperative chemotherapy in patients with confirmed lymph node-positive breast cancer before treatment. Cancer. 2010;116(12):2878-2883. 
19. Carrera D, de la Flor M, Galera J, et al. Validation of sentinel lymph node biopsy in breast cancer women N1-N2 with complete axillary response after neoadjuvant chemotherapy. Multicentre study in Tarragona. Validacion de la biopsia selectiva del ganglio centinela en mujeres con cancer de mama N1-2 con respuesta axilar completa tras la neoadyuvancia. Estudio multicentrico en la provincia de Tarragona. Rev Esp Med Nucl Imagen Mol. 2016;35(4):221-225.

20. Enokido K, Watanabe C, Nakamura S, et al. Sentinel lymph node biopsy after neoadjuvant chemotherapy in patients with an initial diagnosis of cytology-proven lymph node-positive breast cancer. Clin Breast Cancer. 2016;16(4):299-304.

21. Ge WK, Yang B, Zuo WS, et al. Sentinel lymph node biopsy does not apply to all axillary lymph nodepositive breast cancer patients after neoadjuvant chemotherapy. Thorac Cancer. 2014;5(6):550-555.

22. Kang E, Chung IY, Han SA, et al. Feasibility of sentinel lymph node biopsy in breast cancer patients with initial axillary lymph node metastasis after primary systemic therapy. J Breast Cancer. 2011;14(2):147-152.

23. Kuehn T, Bauerfeind I, Fehm T, et al. Sentinel-lymph-node biopsy in patients with breast cancer before and after neoadjuvant chemotherapy (SENTINA): a prospective, multicentre cohort study. Lancet Oncol. 2013;14(7):609-618.

24. Ozmen V, Unal ES, Muslumanoglu ME, et al. Axillary sentinel node biopsy after neoadjuvant chemotherapy. Eur J Surg Oncol. 2010;36(1):23-29.

25. Park S, Park JM, Cho JH, Park HS, Kim SI, Park BW. Sentinel lymph node biopsy after neoadjuvant chemotherapy in patients with cytologically proven node-positive breast cancer at diagnosis. Ann Surg Oncol. 2013;20(9):2858-2865.

26. Pinero-Madrona A, Escudero-Barea MJ, Fernandez-Robayna F, et al. Selective sentinel lymph node biopsy after neoadjuvant chemotherapy in breast cancer: results of the GEICAM 2005-07 study. Cir Esp. 2015;93(1):23-29.

27. Shen J, Gilcrease MZ, Babiera GV, et al. Feasibility and accuracy of sentinel lymph node biopsy after preoperative chemotherapy in breast cancer patients with documented axillary metastases. Cancer. 2007;109(7):1255-1263.

28. Thomas S, Prakash A, Goyal V, Popli MB, Agarwal S, Choudhury M. Evaluation of sentinel node biopsy in locally advanced breast cancer patients who become clinically node-negative after neoadjuvant chemotherapy: a preliminary study. Int J Breast Cancer. 2011;2011:870263.

29. Yagata H, Yamauchi H, Tsugawa K, et al. Sentinel node biopsy after neoadjuvant chemotherapy in cytologically proven node-positive breast cancer. Clin Breast Cancer. 2013;13(6):471-477.

30. Yu Y, Cui N, Li HY, et al. Sentinel lymph node biopsy after neoadjuvant chemotherapy for breast cancer: retrospective comparative evaluation of clinically axillary lymph node positive and negative patients, including those with axillary lymph node metastases confirmed by fine needle aspiration. BMC Cancer. 2016;16(1):808.

31. Zetterlund LH, Frisell J, Zouzos A, et al. Swedish prospective multicenter trial evaluating sentinel lymph node biopsy after neoadjuvant systemic therapy in clinically node-positive breast cancer. Breast Cancer Res Treat. 2017;163(1):103-110.

32. Siso C, de Torres J, Esgueva-Colmenarejo A, et al. Intraoperative ultrasound-guided excision of axillary clip in patients with node-positive breast cancer treated with neoadjuvant therapy (ILINA trial) : A new tool to guide the excision of the clipped node after neoadjuvant treatment. Ann Surg Oncol. 2018;25(3):784-791.

33. Dashevsky BZ, Altman A, Abe H, et al. Lymph node wire localization post-chemotherapy: Towards improving the false negative sentinel lymph node biopsy rate in breast cancer patients. Clin Imaging. 2018;48:69-73.

34. Kim EY, Byon WS, Lee $\mathrm{KH}$, et al. Feasibility of preoperative axillary lymph node marking with a clip in breast cancer patients before neoadjuvant chemotherapy: A preliminary study. World J Surg. 2018;42(2):582-589.

35. Nguyen TT, Hieken TJ, Glazebrook KN, Boughey JC. Localizing the clipped node in patients with nodepositive breast cancer treated with neoadjuvant chemotherapy: Early learning experience and challenges. Ann Surg Oncol. 2017;24(10):3011-3016. 
36. Park S, Koo JS, Kim GM, et al. Feasibility of charcoal tattooing of cytology-proven metastatic axillary lymph node at diagnosis and sentinel lymph node biopsy after neoadjuvant chemotherapy in breast cancer patients. Cancer Res Treat. 2018;50(3):801-812.

37. Plecha D, Bai S, Patterson H, Thompson C, Shenk R. Improving the accuracy of axillary lymph node surgery in breast cancer with ultrasound-guided wire localization of biopsy proven metastatic lymph nodes. Ann Surg Oncol. 2015;22(13):4241-4246.

38. Taback $B$, Jadeja $P, H a$ R. Enhanced axillary evaluation using reflector-guided sentinel lymph node biopsy: A prospective feasibility study and comparison with conventional lymphatic mapping techniques. Clin Breast Cancer. 2018.

39. Fontein DB, van de Water W, Mieog JS, Liefers GJ, van de Velde CJ. Timing of the sentinel lymph node biopsy in breast cancer patients receiving neoadjuvant therapy - recommendations for clinical guidance. Eur J Surg Oncol. 2013;39(5):417-424.

40. van Nijnatten TJ, Schipper RJ, Lobbes MB, Nelemans PJ, Beets-Tan RG, Smidt ML. The diagnostic performance of sentinel lymph node biopsy in pathologically confirmed node positive breast cancer patients after neoadjuvant systemic therapy: A systematic review and meta-analysis. Eur J Surg Oncol. 2015;41(10):1278-1287.

41. NCT01901094. Comparison of axillary lymph node dissection with axillary radiation for patients with node-positive breast cancer treated with chemotherapy. [accessed February 4, 2018]; Available from URL: https://clinicaltrials.gov/ct2/show/NCT01901094.

42. NCT01872975. Standard or comprehensive radiation therapy in treating patients with early-stage breast cancer previously treated with chemotherapy and surgery. [accessed April 13, 2018]; Available from URL: https://clinicaltrials.gov/ct2/show/NCT01872975.

43. van der Noordaa MEM, van Duijnhoven FH, Straver ME, et al. Major reduction in axillary lymph node dissections after neoadjuvant systemic Therapy for node-positive breast cancer by combining PET/CT and the MARI procedure. Ann Surg Oncol. 2018;25(6):1512-1520.

44. Boughey JC, Ballman KV, Le-Petross HT, et al. Identification and resection of clipped node decreases the false-negative rate of sentinel lymph node surgery in patients presenting with node-positive breast cancer (TO-T4, N1-N2) who receive neoadjuvant chemotherapy: Results from ACOSOG Z1071 (Alliance). Ann Surg. 2016;263(4):802-807.

45. van Nijnatten TJA, Simons JM, Smidt ML, et al. A Novel less-invasive approach for axillary staging after neoadjuvant chemotherapy in patients with axillary node-positive breast cancer by combining radioactive iodine seed localization in the axilla with the sentinel node procedure (RISAS): A Dutch prospective multicenter validation study. Clin Breast Cancer. 2017;17(5):399-402.

46. van Nijnatten TJ, Simons JM, Moossdorff M, et al. Prognosis of residual axillary disease after neoadjuvant chemotherapy in clinically node-positive breast cancer patients: isolated tumor cells and micrometastases carry a better prognosis than macrometastases. Breast Cancer Res Treat. 2017;163(1):159-166. 


\section{APPENDIX 4.1}

\section{PubMed search strategy}

("Breast Neoplasms"[Mesh] OR ((carcinoma OR carcinomas OR tumor OR tumours OR tumour OR tumours OR neoplasm OR neoplasms OR malignancy OR adenocarcinoma OR cancer) AND (breast OR mamma))) AND ("Sentinel Lymph Node Biopsy"[Mesh] OR sentinel lymph node biopsy OR slnb OR (MARI[tiab]) OR (Axilla* AND staging[tiab]) OR targeted axillary dissection) AND (sensitivity OR specificity OR "Sensitivity and Specificity"[Mesh] OR "Predictive Value of Tests"[Mesh] OR negative predictive value OR positive predictive value OR likelihood ratio OR diagnosis OR "false-negative" OR "false-positive") AND ("Lymph Nodes"[Mesh] OR "Lymphatic Vessels"[Mesh] OR "Lymphatic Metastasis"[Mesh] OR "Lymphatic System"[Mesh] OR "Axilla"[Mesh] OR axilla*) AND (("Neoadjuvant Therapy"[Mesh] OR neoadjuvant OR preoperative* OR primary) AND ("Antineoplastic Agents"[Mesh] OR chemotherapy OR immunotherapy OR systemic therapy))

\section{EMBASE search strategy*}

((breast OR mamma) AND (cancer OR carcinoma OR neoplasm OR malignancy OR adenocarcinoma OR 'breast cancer'/exp OR tumor OR carcinomas OR tumors OR tumour OR tumours)) AND (('sentinel lymph node biops*' OR slnb OR 'sentinel node*' OR 'sentinel lymph node biopsy'/exp OR 'mari':ab,ti OR (axilla* AND staging) OR 'targeted axillary dissection')) AND (sensitivity OR specificity OR 'predictive value' OR 'likelihood ratio' OR ppv OR npv OR diagnosis OR 'false negative' OR 'false positive') AND ((axilla* OR lymph*) AND (node* OR metastasis)) AND ((neoadjuvant OR primary OR preoperative*) AND (therapy OR immunotherapy OR chemotherapy OR systemic))

*Search results were restricted to articles, reviews, articles in press, conference paper, conference review 


\section{APPENDIX 4.2}

Forest plot of the ax-pCR rate.

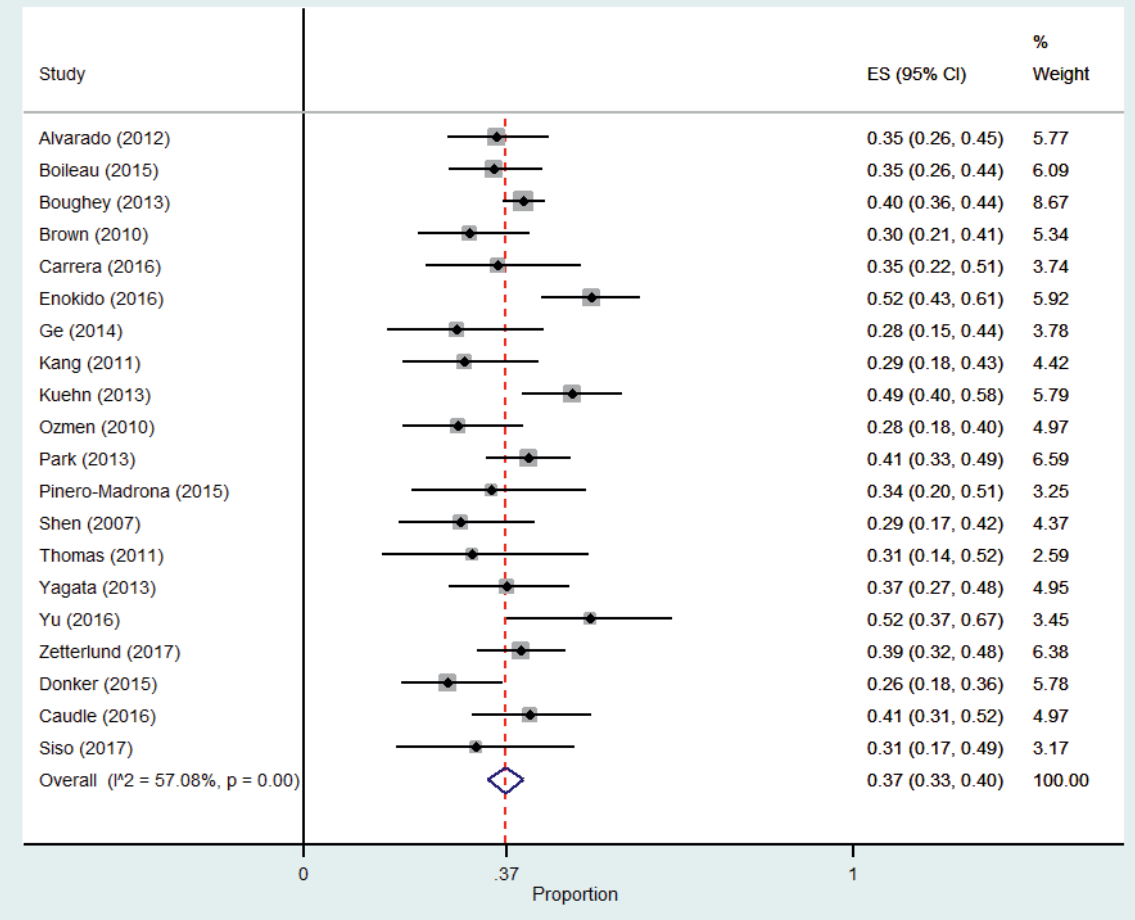

ES: effect size. The pooled ax-pCR is $37 \%$ (33-40\%). 


\section{APPENDIX 3}

Forest plot for the identification rate of SLNB.

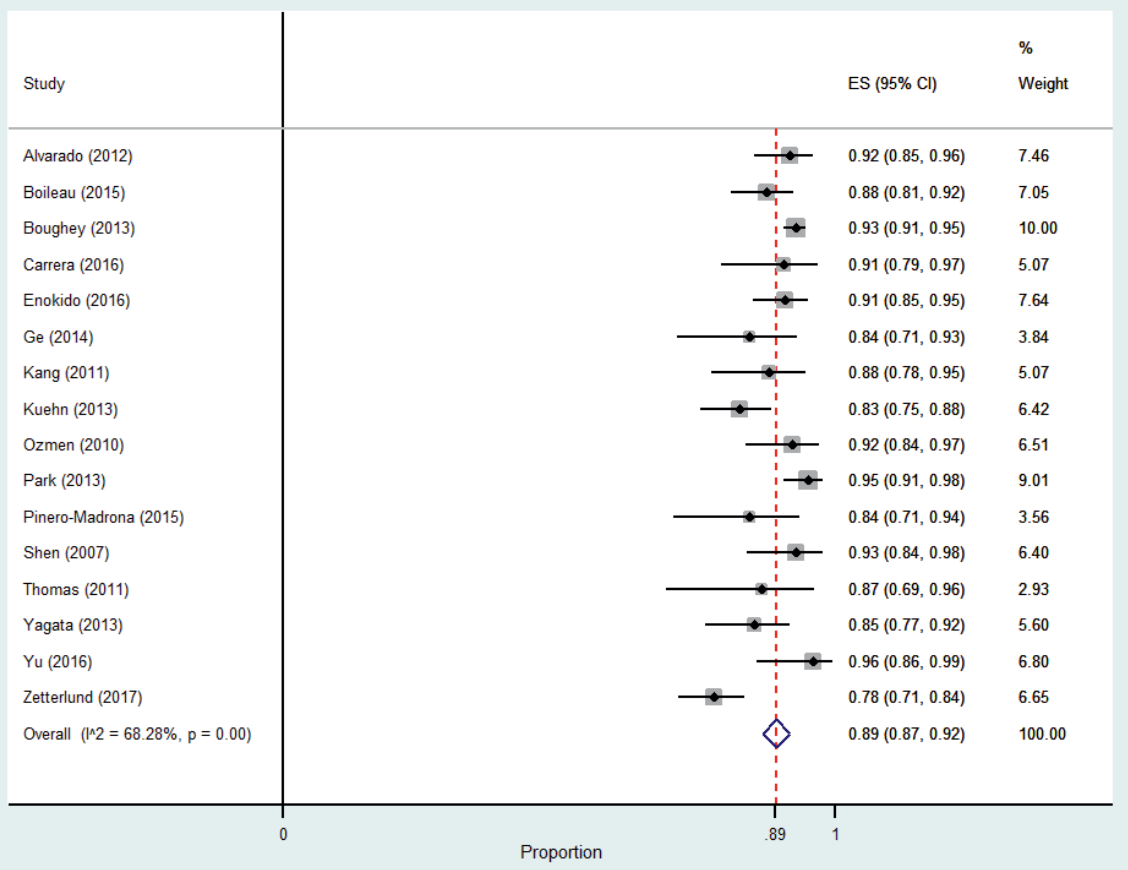

ES: effect size. The pooled identification rate of SLNB is $89 \%$ (87-92\%). 



\section{CHAPTER}

A novel less-invasive approach for axillary staging after neoadjuvant chemotherapy in patients with axillary node-positive breast cancer by combining radioactive iodine seed localization in the axilla with the sentinel node procedure (RISAS): a Dutch prospective multicenter validation study

JM Simons* TJA van Nijnatten* ML Smidt

CC van der Pol

PJ van Diest

A Jager

$B$ van Klaveren

BLR Kam MBI Lobbes

$M$ de Boer

K Verhoef

LB Kloppert*

EJT Luiten*

Clin Breast Cancer. 2017;17(5):399-402

* Authors contributed equally to this work 


\section{ABSTRACT}

\section{Background}

In 1 of 3 patients with initial lymph node-positive $(\mathrm{cN}+)$ breast cancer, neoadjuvant chemotherapy (NAC) results in an axillary pathologic complete response (ax-pCR). This urges the need for a less-invasive axillary staging method. Recently introduced lessinvasive procedures have been insufficient in accurately identifying ax-pCR. Therefore, we propose a novel less-invasive axillary staging procedure: the Radioactive lodine Seed localization in the Axilla with the Sentinel node procedure (RISAS), a combination of the procedure of marking axillary lymph nodes with radioactive iodine seeds (MARI) and sentinel lymph node biopsy (SLNB).

\section{Patients and methods}

In the present open single-arm multicenter validation study, $225 \mathrm{cN}+$ (biopsy-proven) patients will undergo the RISAS procedure, in which a positive lymph node is marked by an iodine-125 seed before NAC. After NAC completion, this iodine-125 seedmarked lymph node is removed, together with any additional sentinel lymph nodes. The RISAS procedure is subsequently followed by completion axillary lymph node dissection (ALND). The RISAS lymph nodes will be compared with the lymph nodes from the completion ALND specimen. The primary endpoint is accuracy of the RISAS procedure. The identification rate, false-negative rate, negative predictive value, and possible concordance between the MARI and SLNB will be reported.

\section{Conclusion}

The present prospective multicenter RISAS trial will enable us to validate the combination of MARI and SLNB for assessing the axillary response to NAC in $\mathrm{CN}+$ patients. If RISAS proves to be an accurate axillary staging procedure, ALND could safely be abandoned in the case of ax-pCR confirmed using the RISAS procedure. 


\section{INTRODUCTION}

Surgical management of the axilla in breast cancer evolved greatly in the past decades. In clinically node negative patients axillary staging has shifted from axillary lymph node dissection (ALND) to sentinel lymph node biopsy (SLNB). In clinically node positive patients $(\mathrm{cN}+)$, ALND is still recommended for axillary staging. ${ }^{1,2}$ However, the shift from adjuvant to neoadjuvant chemotherapy (NAC) has resulted in an increasing need for a less-invasive axillary staging approach, because NAC enables downstaging of axillary disease. ${ }^{3,4}$ In $\leq 36 \%$ of all patients, conversion of $\mathrm{cN}+$ to an axillary pathologic complete response (ax-pCR) as a result of NAC has been reported. ${ }^{5}$ In the case of HER2neu positive patients, ax-pCR can be achieved in $\leq 74 \%$ of patients when NAC is combined with HER2-targeted therapy. ${ }^{6,7}$ Patients in whom ax-pCR is achieved are not expected to benefit from ALND; however, a significant proportion will experience side effects. Consequently, routine ALND is increasingly being regarded as overtreatment in these patients.

To date, imaging techniques have not been able to accurately identify the presence of an ax-pCR. As for surgical techniques, various less-invasive axillary staging procedures have been proposed for $\mathrm{cN}+$ patients after NAC completion. The SLNB seems to be insufficient, with reported false negative rates (FNRs) ranging from $7 \%{ }^{8}$ to $25 \% .{ }^{9}$ Because the SLNB aims to identify patients with an ax-pCR without missing residual disease, a negative predictive value (NPV) of $\geq 95 \%$ would be desirable. A high NPV is especially important since residual tumour is considered resistant to administered chemotherapy and might require additional treatment. However, the NPVs for SLNB have not exceeded $86 \%{ }^{5}$; thus residual axillary disease will be missed in 1 of every 6 patients for whom an ax-pCR is suggested by the $\operatorname{SLN}(\mathrm{s})$. Factors contributing to the improvement of the FNR/NPV of SLNB include using the dual tracer technique and removal of $\geq 3$ SLNs. ${ }^{8,10-12}$ In daily practice, the latter is not always achievable, as was demonstrated by the SENTINA (sentinel neoadjuvant) trial, in which most patients had only 1 to 2 SLNs removed. ${ }^{12}$ Donker et al. ${ }^{13}$ introduced an alternative, less-invasive axillary staging procedure: the MARI procedure (marking axillary lymph nodes with radioactive iodine seeds). In the MARI procedure, an I-125 seed is placed in the biopsy-proven positive axillary lymph node before NAC. After NAC completion, the I-125 seed-containing node is removed at breast surgery. In 98 pretreatment $\mathrm{cN}+$ patients, an identification rate of $97 \%$ and an FNR of $7 \%$ was reported. The MARI procedure has a reported NPV of $83 \%$, indicating that residual axillary disease is still overlooked to the same extent as with the SLNB. ${ }^{13,14}$ In summary, both MARI and SLNB remain insufficiently accurate to use as solitary staging procedures.

Caudle et al. ${ }^{15}$ proposed Targeted Axillary Dissection (TAD), involving clip placement in a biopsy-proven positive axillary lymph node before NAC, followed by localization of 
this clipped node with an I-125 seed after NAC completion. Excision of the I-125 seedmarked node was combined with excision of SLNs. All cN+ patients treated with NAC and in whom the biopsied node was marked with a clip were included in a prospective registry. Retrospectively, 85 patients who had undergone TAD, followed by a completion ALND, were identified. An FNR of $2 \%$ and an NPV of $97 \%$ were reported. $^{15}$ These results are promising; however, the study was hampered by the single-center study design and small sample size, with only 35 patients in whom an ax-PCR was achieved. Consequently, widespread implementation of TAD cannot be recommended before a large, preferably multicenter, validation study has been performed.

Therefore, we propose a multicenter, open, single-arm validation study in which the RISAS (Radioactive lodine Seed localisation in the Axilla combined with the SLNB) procedure is investigated. To validate RISAS, completion ALND will be performed in all patients. The I-125 seed will be placed before the start of NAC. If the results prove that RISAS accurately predicts axillary nodal status after NAC completion, completion ALND and its concomitant morbidity can safely be omitted and prevented in patients with an ax-pCR predicted by RISAS.

\section{PATIENTS AND METHODS}

The RISAS study is an open, single-arm, noninferiority multicenter validation study to assess the accuracy of RISAS for axillary staging after NAC in pretreatment $\mathrm{cN}+$ breast cancer patients. Nodal positivity will always be confirmed by pathologic assessment before the start of NAC in the case of suspicious lymph nodes found on axillary ultrasonogrpahy. Patients will be recruited from approximately eight participating hospitals in the Netherlands during a 2-year period. Each hospital has $\geq$ one year of experience with I-125 seed-guided localization. Given a noninferiority margin of $6.25 \%$ and assuming a prevalence of positive ALND findings of $64 \%$, a total of 225 patients will be needed to test the noninferiority of RISAS compared with ALND.

Patients fulfilling all eligibility criteria will - after completion of NAC - undergo the RISAS procedure and, subsequently, completion ALND (Table 5.1). Patients in whom I-125 seed placement is not possible and in whom the RISAS procedure is not followed by completion ALND will be excluded from final analysis.

The chemotherapy regimens will be determined according to current Dutch breast cancer guidelines ${ }^{1}$ and multidisciplinary team decision making. In HER2neu positive patients, NAC will be combined with HER2-targeted therapy. 
Table 5.1 Eligibility criteria.

\begin{tabular}{l||l} 
Inclusion criteria & Exclusion criteria \\
\hline \hline Female, age $\geq 18$ years & Pregnancy or nursing \\
Invasive breast cancer with biopsy proven positive & Contraindications to RISAS (e.g. allergic reaction to \\
axillary lymph node(s) & blue dye) \\
NAC & $\begin{array}{l}\text { Recurrent breast cancer } \\
\text { History of axillary surgery or radiotherapy } \\
\text { Positive infra- and / or supraclavicular lymph nodes } \\
\text { Distant metastasis }\end{array}$ \\
\hline
\end{tabular}

NAC, neoadjuvant chemotherapy; RISAS, Radioactive lodine Seed localisation in the Axilla combined with the Sentinel node procedure.

The RISAS procedure consists of the MARI procedure combined with SLNB. First, the most suspicious node is sampled using core needle biopsy or fine needle aspiration to confirm nodal positivity. Subsequently, the MARI procedure is performed. This consists of marking the pathologically proven positive lymph node with an I-125 seed before the start of NAC using ultrasound guidance. Retrieval of the sampled node is facilitated by reporting multiple characteristics of th node (eg, diameter of the node and diameter of the cortex). Furthermore, the radiologist will capture an image of the biopsied lymph node. lodine seeds with a maximum activity of $7.4 \mathrm{MBq}(0.2 \mathrm{mCi})$ will be used. ${ }^{16}$ SLNB will be performed using the standard dual tracer technique. After excision of the RISAS nodes, the surgeon will proceed with completion ALND. The RISAS nodes and the remaining nodes of the axillary basin will be secured separately.

The pathologist will assess the RISAS nodes and remaining nodes removed in the completion ALND specimen separately. The presence of isolated tumor cells, micrometastases, and / or macrometastases will be registered. Routine hematoxylin and eosin staining will be performed on all lymph nodes. Immunohistochemistry will only be performed on each RISAS node that is negative on hematoxylin and eosin staining, irrespective of the ALND specimen findings.

The institutional review board of the Erasmus Medical Centre approved the present study (approval no. MEC-2016-412), which was registered at ClinicalTrials.gov (ClinicalTrials.gov identifier, NCT02800317).

Basic statistical analysis will be used. The test outcome of both RISAS and completion ALND can be either negative or positive. If the test outcome of RISAS is positive, the test outcome of the completion ALND will also be considered positive. The outcomes will be formulated in a $2 \times 2$ contingency table. The primary endpoint of our study is accuracy of the RISAS procedure and will be defined by calculating sensitivity, NPV, and FNR. 


\section{DISCUSSION}

With the increasing need for less-invasive axillary staging after NAC, various procedures have been proposed. MARI and SLNB remain insufficient for this purpose, because they miss potentially therapy-resistant disease to a rather high extent. ${ }^{5,11,13}$ TAD has shown promising results; however, owing to the retrospective analysis of patients and small sample size of the single-center study, it seems premature to implement this procedure as standard practice in replacement of ALND. ${ }^{15}$ Our present RISAS study will provide answers to questions regarding TAD, the role of immunohistochemistry, and the appropriate procedure if fewer than 2 nodes will be removed. ${ }^{17}$ An overview of the studies proposing different less-invasive axillary staging procedures is provided in Table 5.2.

Table 5.2 Overview of studies comparing less invasive surgical procedures with axillary lymph node dissection for axillary staging after NAC in pre-treatment $\mathrm{cN}+$ patients.

\begin{tabular}{|c|c|c|c|c|}
\hline & ACOSOG Z107111 & MARI-procedure13 & TAD15 & RISAS \\
\hline Type of procedure & SLNB & $\overline{M A R I}$ & $\begin{array}{l}\text { SLNB + I-125 seed } \\
\text { placement in the } \\
\text { clipped node after } \\
\text { completion of NAC }\end{array}$ & $\begin{array}{c}\text { SLNB }+1-125 \text { seed } \\
\text { placement prior to } \\
\text { NAC }\end{array}$ \\
\hline Sample size* & 687 & 98 & 85 & 225 \\
\hline $\begin{array}{l}\text { Prevalence axillary ax- } \\
\text { pCR }\end{array}$ & 40.0 & 26.0 & 41.0 & \# \\
\hline Identification rate (\%) & 92.7 & 97.0 & 100 & \# \\
\hline FNR (\%) & 14.7 & 7.0 & 2.0 & \# \\
\hline NPV (\%) & 82.0 & 83.0 & 97.2 & \# \\
\hline
\end{tabular}

* This includes all patients in whom concomitant ALND is performed, " pending on results. SLNB sentinel lymph node biopsy, MARI Marking the Axillary lymph node with Radioactive lodine seeds, TAD Targeted Axillary Dissection, RISAS Radioactive lodine seed localisation in the Axilla combined with Sentinel node procedure, I-125 seed iodine-125 seed, NAC neoadjuvant chemotherapy, $a x-p C R$ pathologic complete response, FNR false-negative rate, NPV negative predictive value.

Because RISAS combines MARI and SLNB, the accuracy of RISAS is thought to not be hindered by the shortcomings of the solitary techniques. Metastatic lymph nodes can block and deviate normal lymphatic drainage, resulting in "false SLNs". Given that the RISAS procedure not only retrieves (false) SLNs but also pretreatment pathologically proven metastatic lymph nodes, it has the potential to be equal to ALND in staging of the axilla. Caudle et al. ${ }^{15}$ have already demonstrated that the clipped node was not retrieved as an SLN in $23 \%$ of patients.

An advantage of RISAS compared with TAD is that the biopsy-proven positive node is primarily marked by an I-125 seed and not preceded by clip placement. The exact placement of the I-125 seed directly adjacent to the clip might be challenging and, 
thus, could possibly hamper accuracy. Furthermore, direct marking of the lymph node by an I-125 seed obviates an extra hospital visit after NAC completion, which minimizes patient burden and obviates an additional procedure, reducing logistical issues.

\section{Conclusion}

The RISAS study is a multicenter prospective noninferiority study to validate the combination of MARI and SLNB for predicting the axillary response to NAC in pretreatment $\mathrm{cN}+$ patients. The ultimate goal is to identify ax-pCR patients using a minimally invasive procedure to prevent unnecessary ALND, with its associated side effects, without comprising oncologic safety. ALND could then become a management procedure reserved for patients with residual axillary disease.

\section{ACKNOWLEDGMENTS}

The present study received grant support from the Alpe d'Huzes/Dutch Cancer Society (KWF Kankerbestrijding) fund (grant AZB 2015-8023). 


\section{REFERENCES}

1. Richtlijn Mammacarcinoom, NABON 2012.

2. National Comprehensive Cancer Network. NCCN clinical practice guidelines in oncology: breast cancer. V.1.2015.

3. Boughey JC, McCall LM, Ballman KV, et al. Tumor biology correlates with rates of breast-conserving surgery and pathologic complete response after neoadjuvant chemotherapy for breast cancer: findings from the ACOSOG Z1071 (Alliance) Prospective Multicenter Clinical Trial. Ann Surg. 2014;260(4):608-614; discussion 614-606.

4. Cortazar P, Zhang L, Untch M, et al. Pathological complete response and long-term clinical benefit in breast cancer: the CTNeoBC pooled analysis. Lancet. 2014;384(9938):164-172.

5. van Nijnatten TJ, Schipper RJ, Lobbes MB, Nelemans PJ, Beets-Tan RG, Smidt ML. The diagnostic performance of sentinel lymph node biopsy in pathologically confirmed node positive breast cancer patients after neoadjuvant systemic therapy: A systematic review and meta-analysis. Eur J Surg Oncol. 2015;41(10):1278-1287.

6. Dominici LS, Negron Gonzalez VM, Buzdar AU, et al. Cytologically proven axillary lymph node metastases are eradicated in patients receiving preoperative chemotherapy with concurrent trastuzumab for HER2-positive breast cancer. Cancer. 2010;116(12):2884-2889.

7. van la Parra RF, Kuerer HM. Selective elimination of breast cancer surgery in exceptional responders: historical perspective and current trials. Breast Cancer Res. 2016;18(1):28.

8. Mamounas EP, Brown A, Anderson S, et al. Sentinel node biopsy after neoadjuvant chemotherapy in breast cancer: results from National Surgical Adjuvant Breast and Bowel Project Protocol B-27. J Clin Oncol. 2005;23(12):2694-2702.

9. Shen J, Gilcrease MZ, Babiera GV, et al. Feasibility and accuracy of sentinel lymph node biopsy after preoperative chemotherapy in breast cancer patients with documented axillary metastases. Cancer. 2007;109(7):1255-1263.

10. Boileau JF, Poirier B, Basik M, et al. Sentinel node biopsy after neoadjuvant chemotherapy in biopsyproven node-positive breast cancer: the SN FNAC study. J Clin Oncol. 2015;33(3):258-264.

11. Boughey JC, Suman VJ, Mittendorf EA, et al. Sentinel lymph node surgery after neoadjuvant chemotherapy in patients with node-positive breast cancer: the ACOSOG Z1071 (Alliance) clinical trial. JAMA. 2013;310(14):1455-1461.

12. Kuehn T, Bauerfeind I, Fehm T, et al. Sentinel-lymph-node biopsy in patients with breast cancer before and after neoadjuvant chemotherapy (SENTINA): a prospective, multicentre cohort study. Lancet Oncol. 2013;14(7):609-618.

13. Donker M, Straver ME, Wesseling J, et al. Marking axillary lymph nodes with radioactive iodine seeds for axillary staging after neoadjuvant systemic treatment in breast cancer patients: the MARI procedure. Ann Surg. 2015;261(2):378-382.

14. Vugts G, Nieuwenhuijzen GA, Maaskant-Braat AJ, Schipper RJ, Smidt ML. Axillary response monitoring after neoadjuvant chemotherapy in breast cancer: Can we avoid the morbidity of axillary treatment? Ann Surg. 2016;263(2):e28-29.

15. Caudle AS, Yang WT, Krishnamurthy S, et al. Improved axillary evaluation following neoadjuvant therapy for patients with node-positive breast cancer using selective evaluation of clipped nodes: implementation of targeted axillary dissection. J Clin Oncol. 2016;34(10):1072-1078.

16. Straver ME, Loo CE, Alderliesten T, Rutgers EJ, Vrancken Peeters MT. Marking the axilla with radioactive iodine seeds (MARI procedure) may reduce the need for axillary dissection after neoadjuvant chemotherapy for breast cancer. Br J Surg. 2010;97(8):1226-1231.

17. Fedor DM, Landercasper J. Can a seed-sized tool from Texas spare clinically node positive breast cancer patients from a complete axillary dissection? Gland Surg. 2016;5(4):450-452. 




\section{CHAPTER}

Excision of both the pretreatment marked positive node and sentinel nodes improves axillary staging after neoadjuvant systemic therapy study

JM Simons

MLMA van Pelt

AWKS Marinelli

ME Straver

AM Zeillemaker LM Pereira Aris-Bouda

TJA van Nijnatten

LB Kloppert KK Hunt

EJT Luiten

CC van der Pol

Br J Surg. 2019 Oct. Doi: 10.1002/bjs.11320 


\section{ABSTRACT}

\section{Background}

Marking the axilla with radioactive iodine seed and sentinel lymph node (SLN) biopsy have been proposed for axillary staging after neoadjuvant systemic therapy in clinically node-positive breast cancer. This study evaluated the identification rate and detection of residual disease with combined excision of pretreatment-positive marked lymph nodes (MLNs) together with SLNs.

\section{Methods}

This was a multicentre retrospective analysis of patients with clinically node-positive breast cancer undergoing neoadjuvant systemic therapy and the combination procedure (with or without axillary lymph node dissection). The identification rate and detection of axillary residual disease were calculated for the combination procedure, and for MLNs and SLNs separately.

\section{Results}

At least one MLN and/or SLN(s) were identified by the combination procedure in 138 of 139 patients (identification rate $99.3 \%$ ). The identification rate was $92.8 \%$ for MLNs alone and $87.8 \%$ for SLNs alone. In 88 of 139 patients (63.3\%) residual axillary disease was detected by the combination procedure. Residual disease was shown only in the MLN in 20 of 88 patients (23\%) and only in the SLN in ten of $88(11 \%)$, whereas both the MLN and SLN contained residual disease in the remainder (58 of $88,66 \%)$.

\section{Conclusion}

Excision of the pretreatment-positive MLN together with SLNs after neoadjuvant systemic therapy in patients with clinically node-positive disease resulted in a higher identification rate and improved detection of residual axillary disease. 


\section{INTRODUCTION}

Over the past few decades, there has been a trend towards de-escalation of surgical management of the axilla. In patients with clinically node-positive $(\mathrm{cN}+)$ disease, axillary lymph node dissection (ALND) is still performed frequently, providing both regional control and information for adjuvant therapy recommendations. Neoadjuvant systemic therapy (NST) is often given to patients with $\mathrm{cN}+$ disease, leading to a pathological complete response ( $p C R)$ in the axilla in approximately onethird of patients. ${ }^{1}$ Patients with an axillary pCR do not benefit from ALND, yet do suffer from both short- and long-term side-effects of the operation. There is a need for less invasive axillary staging methods for these patients.

Various less invasive procedures have been proposed for axillary staging after NST in patients with $\mathrm{cN}+$ tumours before treatment. However, neither the sentinel lymph node (SLN) nor the marking the axilla with a radioactive iodine seed (MARI) procedure have low enough false-negative rates (FNRs) for these techniques to comfortably replace ALND. ${ }^{1-4}$ Based on the negative predictive values (NPVs) of these procedures, residual axillary disease may be missed in at least one in six patients with $\mathrm{cN}+$ disease in whom an axillary pCR is suggested..$^{5-7}$ To improve accuracy, Caudle and colleagues ${ }^{8}$ introduced targeted axillary dissection, which is a combination of SLN biopsy (SLNB) and a MARI-like procedure; this was shown to have a FNR of $2 \%$ and a NPV of $97 \%$ in a cohort of 85 patients. Based on this, residual axillary disease would be missed in only one of 33 patients in whom an axillary PCR is suggested. The ongoing Dutch RISAS trial (NCT02800317) $)^{9}$ will assess whether these promising results of a combination procedure can be validated in a prospective multicentre study.

In the absence of high-level evidence, various protocols involving less invasive axillary staging are being implemented in clinical practice. Those in favour of SLNB alone believe that pretreatment marking of the positive lymph node is an unnecessary extra procedure, and instead support the removal of at least three sentinel nodes to improve accuracy. Advocates of the MARI procedure believe that SLNB is not of additional benefit if removal of the marked lymph node (MLN) is guaranteed, whereas others stress the need to combine these procedures to secure accurate staging. At the same time, omission of ALND is controversial as long-term follow-up of patients with $\mathrm{cN}+$ disease in whom ALND is omitted is not yet available.

This large multicentre retrospective study analysed a cohort of patients with $\mathrm{cN}+$ breast cancer who underwent a combination procedure after NST. The combination procedure comprised excision of both a pretreatment-positive MLN and SLN(s) instead of performing standard ALND for axillary staging after NST. The identification 
rate and detection of residual disease of the combination procedure, and its advantages over either the MARI procedure or SLNB alone, are reported.

\section{METHODS}

This retrospective cohort study included patients with pathologically proven nodepositive breast cancer who underwent a combination procedure with excision of the MLN and SLN(s) after NST. Patients with distant metastasis and those who underwent SLNB before NST were not eligible. Patients were treated between September 2014 and November 2017 in four hospitals in the Netherlands: University Medical Centre Utrecht in Utrecht, Amphia Hospital in Breda, Haaglanden Medical Centre in The Hague and Alrijne Hospital in Leiderdorp. Multidisciplinary tumour boards at these centres reviewed local protocols for axillary staging, which led to replacing or preceding ALND with excision of the MLN and SLN(s) after NST in patients with cN+ disease. Type of adjuvant axillary surgery and/or radiation therapy was also decided by the multidisciplinary tumour boards. Medical records were obtained to collect data on age, breast cancer subtype, receptor status, TNM classification (AJCC cancer staging manual, $7^{\text {th }}$ Edition, 2010) before and after NST, NST regimens, imaging findings, radiological and surgical procedures, and adjuvant treatment.

This study protocol was reviewed by the Medical Research Ethics Committee of University Medical Centre Utrecht (number 18/111); the requirement for written informed consent was waived.

\section{Neoadjuvant systemic therapy}

Systemic therapy regimens were determined according to the Dutch breast cancer guidelines (2012) and local multidisciplinary tumour board preferences. In human epidermal growth factor receptor 2 (HER2)-positive disease, NST was combined with HER2-targeted therapy.

\section{Pretreatment marking of positive lymph node}

Suspicious lymph nodes identified on imaging were examined pathologically by sampling the (most) suspicious lymph node using fine-needle aspiration or core needle biopsy. Subsequently, the same lymph node, if proven $\mathrm{N}+$, was marked by a radiologist under ultrasound guidance before the start of NST by means of either an iodine seed or a radio-opaque clip, depending on local practice. If a clip was used, a wire or iodine seed was placed within the clipped lymph node after completion of NST, to facilitate removal of the clipped node during surgery. If there were multiple suspicious lymph nodes, only one node was biopsied, and marked if positive. 


\section{Sentinel lymph node biopsy}

SLNB was performed by a single-tracer method ( $\left.{ }^{99 m} \mathrm{Tc}\right)$ in Haaglanden Medical Centre, and by a dual-tracer method (Tc and blue dye) in the other three centres. In the event of negative lymphoscintigraphy, Tc was reinjected before surgery depending on local protocols. In some patients, sampling with blue dye alone was performed. Suspicious and/or enlarged non-SLN(s) were removed at the discretion of the surgeon.

\section{Surgery}

The combination procedure was performed simultaneously with removal of the breast by a dedicated breast surgeon. The MLN containing the iodine seed was excised under guidance of a hand-held probe set to detect ${ }^{125} \mathrm{I}$. If the MLN contained a wire, the wire was used to guide excision of the MLN. The probe was then set to detect Tc and used to identify SLN(s). Surgeons were trained to note whether the MLN also showed Tc radioactivity or blue dye uptake, that is whether the MLN was the sentinel node. In all patients with an iodine seed, excision of this seed was confirmed by means of a specimen radiograph and/or the absence of ${ }^{125} \mathrm{I}$ radioactive counts in the axilla. Specimen radiography was not undertaken routinely in patients who had wire localization.

\section{Pathology}

All lymph nodes were sectioned and stained with haematoxylin and eosin, and the pathologist could opt to use immunohistochemical analysis of the MLN and/or SLN(s). The following items were reported: number of lymph nodes, presence of an iodine seed or clip, number of positive lymph nodes and extent of residual disease. The number of examined lymph nodes reported by the pathologist was documented for the MLN and SLN separately. Their sum was the number of examined lymph nodes for the combination procedure, unless the MLN was the sentinel node. The pathological outcome of the combination procedure was based on the combined outcome of the MLN and SLNB (Table S6.1, supporting information).

\section{Adjuvant treatment}

Adjuvant treatment was based on national guidelines and local multidisciplinary tumour board preferences. As residual disease may have been missed by the combination procedure in a limited number of patients, adjuvant axillary radiotherapy was frequently recommended, and also in the event of an axillary pCR. The need for completion ALND was determined on an individual basis. 


\section{Statistical analysis}

The aim was to report on experiences with the combination procedure and not the accuracy of the combination procedure, as completion ALND was not undertaken in all patients. The focus was therefore on identification rate and detection of axillary residual disease for the MLN, SLNB and the combination procedure. In terms of identification rate, the combination procedure was considered successful if at least one lymph node (MLN and/or SLN) was identified. Regarding the detection of axillary residual disease, it was determined if the ML and the SLN were one and the same node based on data from the surgery and/or pathology report.

Either the $X^{2}$ test or Fisher's exact test was used to compare unpaired data. The McNemar exact test was used for analysis of paired assessments of the proportion of patients with $\mathrm{cN}+$ disease in whom residual axillary disease was detected by the MLN, SLN(s) or by the combination of MLN with SLN(s). Statistical analysis was performed using SPSS ${ }^{\oplus}$ for Windows version 24 (IBM, Armonk, New York, USA).

\section{RESULTS}

A total of 139 patients from four institutions were included in this study (Figure 6.1). Patient, tumour and treatment characteristics are shown in Tables 6.1 and 6.2. An overall axillary pCR was identified in 50 of 139 patients $(36.0 \%)(74 \%$ for patients with HER2-positive, $44 \%$ for those with triple-negative, and $7.4 \%$ for patients with hormone receptor-positive, HER2-negative tumours respectively), based on final pathological assessment of MLN, SLNs and, if applicable, completion ALND. A breast pCR was identified in 48 of 139 patients (34.5\%), and a pCR in both the breast and axilla in 40 of $139(28.8 \%)$.

\section{Preoperative details of nodal assessment}

Data on the number of suspicious lymph nodes before NST was available for 130 of 139 patients (93.5\%). The median number of suspicious lymph nodes identified on ultrasound examination was 1 (range 0-5). The median number identified by MRI and/or PET-CT was 2 (range 0-9), with data available for 126 of 139 patients (90.6\%).

The MLN was marked primarily with an iodine seed in 68 of 139 patients (48.9\%) and with a clip in 71 (51.1\%). When a clip was used, a wire (58 of $71,82 \%$ ) or iodine seed (12 of $71,17 \%$ ) was placed within the lymph node after completion of NST. In one patient $(1 \%)$, placement of a wire was not attempted owing to the location of the lymph node and the risk of pneumothorax.

Lymphoscintigraphy was undertaken in 131 of 139 patients $(94.2 \%)$ as part of the SLNB procedure, and one or more hotspots were identified in 104 of 131 (79.4\%). 
SLNB was performed using a dual-tracer technique in 76 of 139 patients (54.7\%) and a single-tracer technique in $63(45.3 \%) ; 55$ of the latter patients $(87 \%)$ patients had lymphoscintigraphy with Tc only and eight (13\%) with blue dye only.

Figure 6.1 Flow chart for the study.

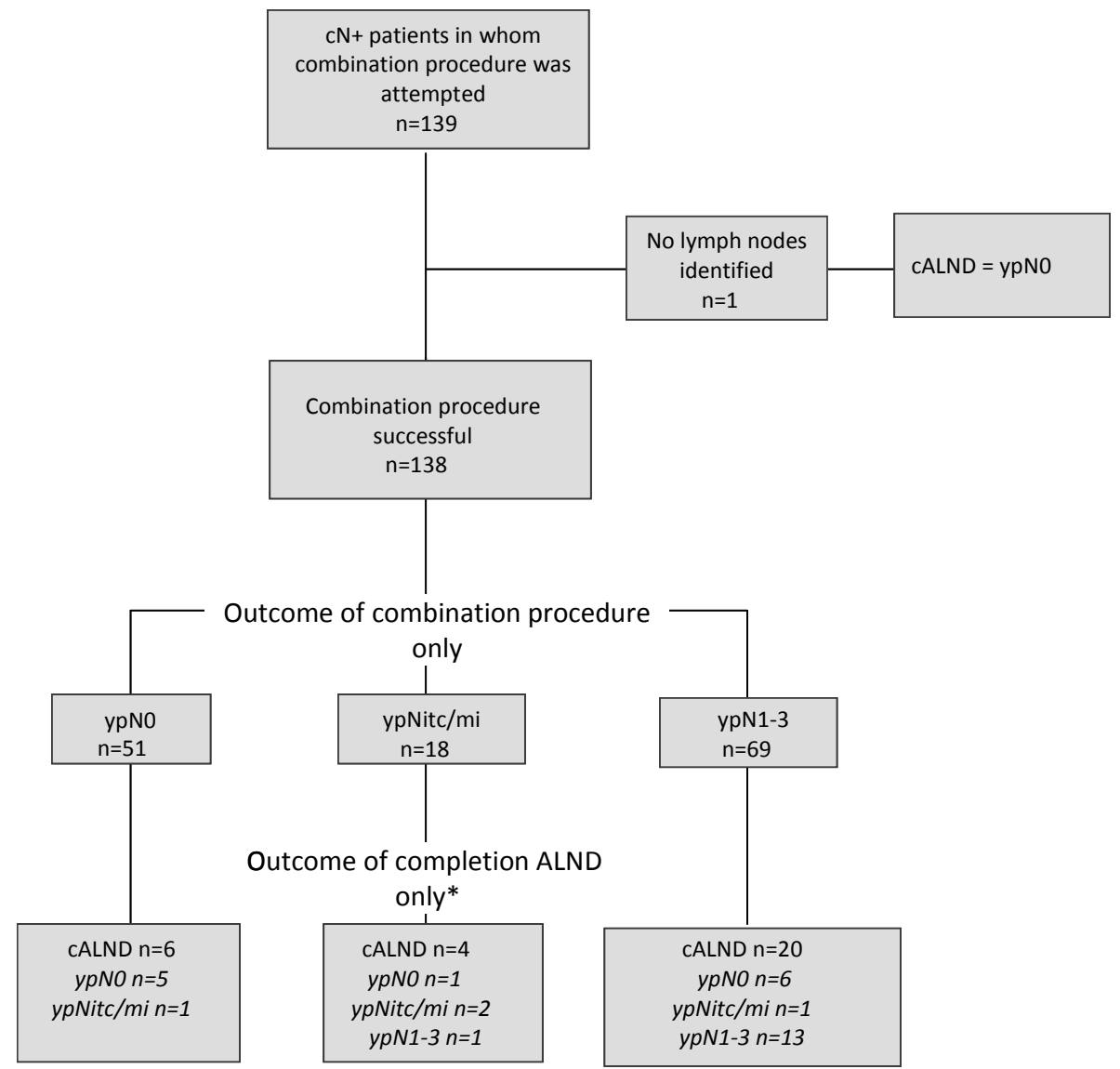

* If completion ALND was performed. CALND; completion ALND. cALND, completion axillary lymph node dissection; ITC, isolated tumor cells; mi, micrometastases. 
Table 6.1 Patient and tumour characteristics among patients with clinically node-positive disease.

\begin{tabular}{|c|c|}
\hline Characeteristic & $\begin{array}{l}\text { No. of patients* } \\
(n=139)\end{array}$ \\
\hline $\begin{array}{l}\text { Treating centre } \\
\text { University Medical Centre Utrecht } \\
\text { Amphia Hospital } \\
\text { Medical Centre Haaglanden } \\
\text { Alrijne Hospital } \\
\end{array}$ & $\begin{array}{l}23(16.5) \\
22(15.8) \\
59(42.4) \\
35(25.2)\end{array}$ \\
\hline Age (years) ${ }^{\dagger}$ & $56(26-82)$ \\
\hline $\begin{array}{l}\text { Clinical tumour category } ¥ \\
\text { cT1 } \\
\text { cT2 } \\
\text { cT3 } \\
\text { cT4 }\end{array}$ & $\begin{array}{l}19(14) \\
78(57.4) \\
27(19.9) \\
12(8.8)\end{array}$ \\
\hline $\begin{array}{l}\text { Clinical node category } \\
\text { cN1 } \\
\text { cN2 } \\
\text { cN3 }\end{array}$ & $\begin{array}{r}102(73.4) \\
26(18.7) \\
11(7.9)\end{array}$ \\
\hline $\begin{array}{l}\text { Histology } \\
\text { Ductal } \\
\text { Lobular } \\
\text { Ductulolobular } \\
\text { Other§ }\end{array}$ & $\begin{array}{l}117(84.2) \\
10(7.2) \\
7(5.0) \\
5(3.6)\end{array}$ \\
\hline $\begin{array}{l}\text { Molecular subtype } \\
\text { HR+/HER2+ } \\
\text { HR-/HER2+ } \\
\text { HR+/HER2- } \\
\text { Triple-negative } \\
\end{array}$ & $\begin{array}{l}24(17.3) \\
22(15.8) \\
68(48.9) \\
25(18.0) \\
\end{array}$ \\
\hline $\begin{array}{l}\text { Method of confirmation of nodal positivity } \\
\text { FNAC } \\
\text { CNB }\end{array}$ & $\begin{array}{c}126(90.6) \\
13(9.4)\end{array}$ \\
\hline
\end{tabular}

* With percentages in parentheses are unless indicated otherwise; † values are median (range). ¥ Data available for 136 patients; one patient had relapse in mastectomy scar (patient A), one had ductal carcinom in situ after neoadjuvant systemic therapy (NST) but no histopathological diagnosis before NST (patient B), and one had axillary relapse without signs of local relapse (patient $\mathrm{C}$ ). § Tubulolobular carcinoma in one patient, tubular carcinoma in one patient, data missing for three patients (including patients B and C). HR, hormone receptor; HER2, human epidermal growth factor receptor 2; FNAC, fine-needle aspiration cytology; CNB, core needle biopsy.

\section{Identification rates}

The MLN procedure had an overall identification rate of $92.8 \%$ (129 of 139). The identification rate was $93 \%$ both for primary marking with an iodine seed and with a clip ( $p=0.994)$. In patients with a clip, the identification rate was $93 \%$ (55 of 58 ) for patients with a clip-wire combination and 95\% (11 of 12) for those with a clip-seed combination $(p=0.668)$. The iodine seed was retrieved in all patients, but a lymph node was not identified in six, meaning that the iodine seed was not located within a lymph node but in the adjacent adipose tissue. SLN(s) were identified in nine of ten patients in whom the MLN was not identified. 
Table 6.2 Treatment characteristics.

\begin{tabular}{l||c} 
Characteristic & $\begin{array}{c}\text { No. of patients } \\
\text { ( } \boldsymbol{n}=139)\end{array}$ \\
\hline \hline Neoadjuvant regimen & $82(59.0)$ \\
Chemotherapy only & $44(31.7)$ \\
Chemotherapy + HER2-directed therapy & $13(9.4)$ \\
Endocrine therapy only & $68(48.9)$ \\
\hline Type of pretreatment lymph node marker & $71(51.1)$ \\
lodine seed & $87(63.5)$ \\
Clip & $50(36.5)$ \\
\hline Breast surgery* & $108(77.7)$ \\
Breast-conserving surgery & $31(22.3)$ \\
Mastectomy & \\
\hline Axillary surgery & \\
Combination procedure only & \\
Combination procedure + completion ALND & \\
\hline
\end{tabular}

Values in parentheses are percentages. * Excluding patients $A$ and $C$ in Table 6.1. HER2, human epidermal growth factor receptor 2; ALND, axillary lymph node dissection.

The SLNB procedure had an overall identification rate of $87.8 \%$ (122 of 139). The identification rate was $86 \%$ for the dual-tracer and $90.4 \%$ for the single-tracer technique $(p=0.375)$. Three or more SLNs were removed from 46 of 122 patients (37.7\%). An MLN was identified in 16 of 17 patients in whom no SLN(s) were identified.

The combination procedure had an overall identification rate of $99.3 \%$ (138 of 139). At least one MLN and/or SLN was identified in 138 patients. No nodes were retrieved from one patient because no SLN was identified during surgery and the iodine seed was not located within or adjacent to a lymph node. Completion ALND in this patient showed an axillary $\mathrm{pCR}$. The median number of lymph nodes resected with the combination procedure was 2 (mean 2.6; range 1-9, i.q.r. 1-3). Both the MLN and SLN(s) were identified in 113 of 139 patients (81.3\%); either the MLN alone (16 of 139, $11.5 \%$ ) or only the SLN(s) (9 of 139, 6.5\%) were identified in the remaining 25 patients (Figure 6.2). Whether the MLN and the sentinel node were the same was reported for 96 of 113 patients (85.0\%), and this was the case in 62 of 96 (65\%).

\section{Detection of residual axillary disease with the combination procedure}

Residual axillary disease was detected in 88 of 139 patients (63.3\%) with the combination procedure. The median number of lymph nodes resected with the combination procedure was 2 (range 1-9) among patients with and 2 (1-7) in those without residual disease. Both the MLN and SLN(s) contained residual disease in 58 of 88 patients (66\%). Residual disease was detected only in the MLN in 20 of 88 (23\%); no $\operatorname{SLN}(\mathrm{s})$ were identified in 11 of these patients, and in nine patients the SLN did not 
contain residual disease whereas the MLN did. Residual disease was detected only in the SLN(s) in ten of 88 patients (11\%); no MLN was identified in one patient, and in nine patients the MLN did not contain residual disease but the SLN(s) did (Figure 6.3). The proportion of all patients in whom residual axillary disease was identified by the combination procedure (MLN and SLN(s)) was $63.3 \%$ and this was significantly higher than the proportion identified using either MLN (56.1\%; $p=0.002)$ or SLN (48.9\%; $\mathrm{p}<0.001)$ procedures alone. The proportion of patients in whom residual axillary disease was identified did not differ significantly between the MLN and the SLN procedures (56.1 versus 48.9\%; $\mathrm{p}=0.100$ ).

Palpable or suspicious lymph nodes were removed in addition to the MLN and/or SLN(s) in 29 of 139 patients (20.9\%) at the discretion of the surgeon. Additional nodes were removed in 21 of 122 patients (17.2\%) with SLNs identified, and in eight of 17 (47\%) with no SLNs identified. The additionally removed nodes showed macrometastasis, whereas the MLN and/or SLN(s) were negative, in three of 29 patients. In the remaining 26 patients, the additional nodes did not change the outcome based on the MLN and/or SLN(s); the disease was classified as ypN+ in 15 patients and as ypNO in 11.

Figure 6.2 Success rate in identifying both the marked lymph node and sentinel lymph nodes in a patient.

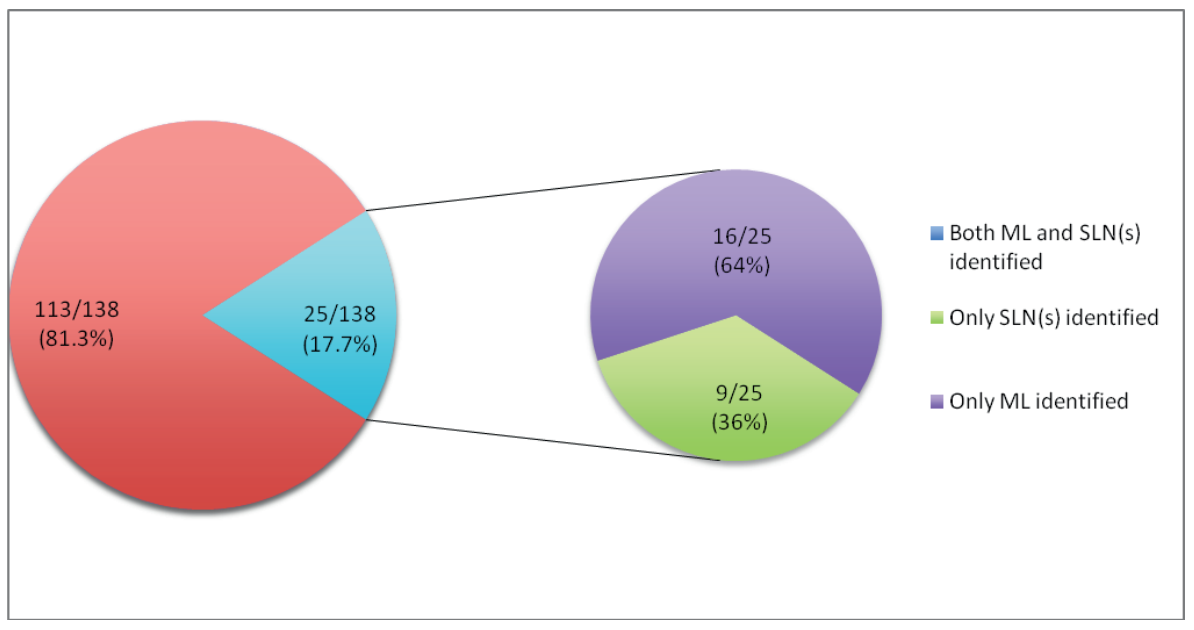

The figure represents 138 of 139 patients, as no marked lymph node (MLN) or sentinel lymph node (SLN) was identified in one patient. Patients in whom the MLN and SLN were one and the same were included in the group with both MLN and SLN(s) identified. In 25 of 138 patients, it was not possible to identify both the MLN and SLNs, but either the MLN or SLN(s) was identified. 
Figure 6.3 Types of node in which disease was found among patients with residual axillary disease identified by the combination procedure.

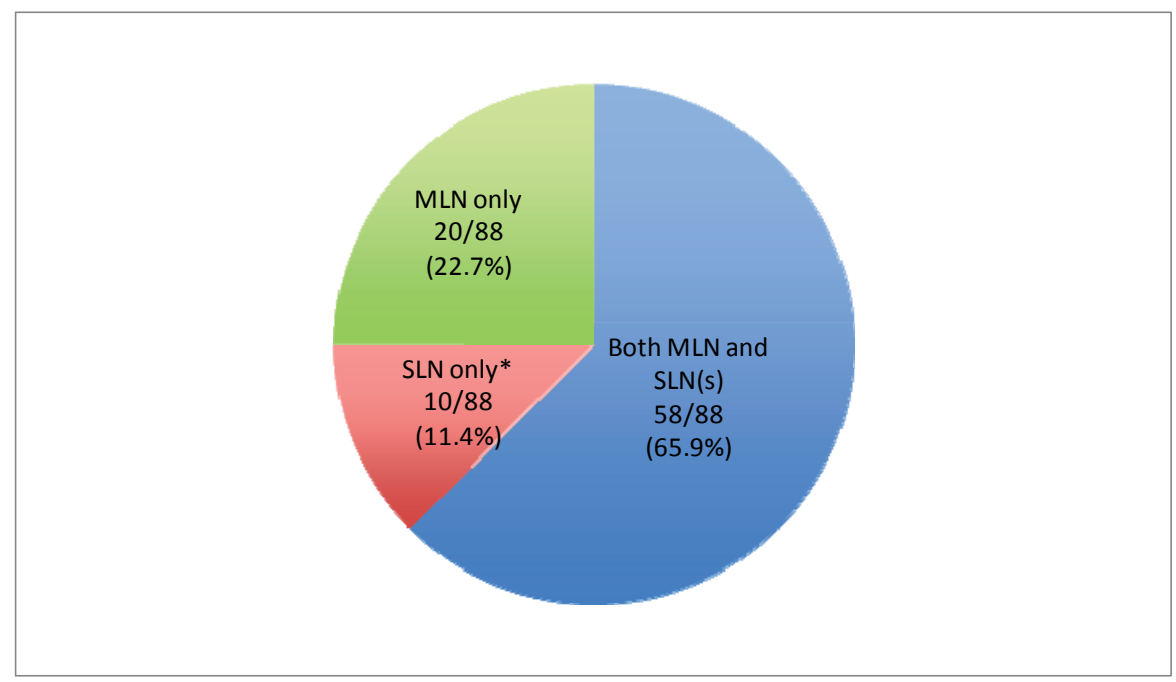

The population comprises all 88 patients in whom residual axillary disease was detected by the combination procedure. Residual disease was found only in the marked lymph node (MLN) and not in the sentinel lymph node (SLN) (either because no SLN was identified or because the SLN was free from disease), only in the $\operatorname{SLN}(s)$ and not in the MLN (either because no MLN was identified or because the MLN was free from disease) or in the MLN as well as the SLN(s). *Including palpable non-SLN(s) if applicable.

\section{Adjuvant axillary radiotherapy}

Adjuvant radiotherapy treatment plans were available for 138 of 139 patients (99.3\%). Adjuvant radiotherapy of the axilla was planned in 114 of 138 patients (82.6\%), including the periclavicular nodes in $74.6 \%$ and including internal mammary nodes in $2.6 \%$.

\section{DISCUSSION}

This retrospective analysis of a large multicentre cohort of patients who presented with biopsy-confirmed $\mathrm{cN}+$ disease evaluated the identification rate and detection of residual disease of the MLN in combination with SLNB after NST. This less invasive combination procedure had an excellent identification rate of $99.3 \%$, and enabled improved detection of residual axillary disease compared with either the MLN alone or the SLNB procedure alone. 
The optimal staging and management of the axilla in patients with $\mathrm{cN}+$ disease who receive NST is controversial. The need for consensus on the most appropriate method for axillary staging in this situation is reflected by the varying practices worldwide. Although SLNB has proven accurate in patients with clinically node-negative disease, both before and after $\mathrm{NST}^{10}$, it is associated with less favourable accuracy in those with $\mathrm{cN}+$ tumours treated with NST. The St Gallen International Expert Consensus Conference $^{11}$ on axillary surgery after NST considered SLNB to be adequate for axillary staging before NST in patients with $\mathrm{cN}+$ disease. The recommendation for patients with a clinically positive axilla after NST, and with a limited number of SLN(s) resected (fewer than 3) or with macrometastatic disease identified in the SLNs, remains ALND. It has been reported that the accuracy of SLNB in patients with $\mathrm{cN}+$ tumours treated with NST depends on the number of excised $\operatorname{SLN}(\mathrm{s})$ and whether the dual-tracer technique is used. Although the dual-tracer technique was used in only $54.7 \%$ of patients in the present cohort, the identification rate for SLNB was high at $87.8 \%$. However, only $37.7 \%$ had three or more SLNs removed. To act in accordance with the St Gallen recommendations would have required completion ALND in a significant number of patients, including those with an axillary pCR. As most patients will not have at least three SLNs identified at surgery, ensuring removal of the MLN in addition to the SLN(s) may be a better strategy. Targeted axillary dissection, developed at MD Anderson Cancer Center, appears promising, with a FNR of $2 \%$ and NPV of $97 \%{ }^{8}$ Recently, the prospective ILINA trial ${ }^{12}$ was reported, which included ultrasoundguided excision of the MLN in combination with SLNB. Of 46 patients with $\mathrm{cN}+$ disease treated with NST, 35 completed the protocol followed by ALND, resulting in a FNR of $4.1 \%$ and NPV of $91.7 \%$. Although both studies reported promising results, evidence is hampered by single-centre study designs and small sample sizes. The Dutch multicentre RISAS trial ${ }^{6}$ is currently accruing with the aim of enrolling a prospective cohort of 225 patients with $\mathrm{cN}+$ disease.

A survey ${ }^{13}$ among members of the American Society of Breast Surgeons revealed that only $15 \%$ of 638 respondents still perform ALND in all patients with $\mathrm{cN}+$ tumours treated with NST, and that 54\% offer SLNB with possible omission of ALND in over half of their patients. The survey also showed that different methods are used for localization of the clipped and marked lymph node (73\% wire, $13 \%$ iodine seed, $14 \%$ other) after completion of NST. In the present cohort, identification rates were similar for patients in whom the positive lymph node was marked primarily with an iodine seed versus a clip. For patients in whom a clip was placed, identification rates were also similar between placing a wire or an iodine seed after NST. Other marking techniques reported previously, such as charcoal tattooing ${ }^{14,15}$, were not used here. Besides using a combination of the MLN and $\operatorname{SLN}(s)$, recent reports ${ }^{16,17}$ have suggested combining the outcome of MARI with the number of fluorodeoxyglucose-avid lymph 
nodes on PET-CT carried out before NST to determine the need for further axillary treatment. Among 159 patients with $\mathrm{cN}+$ disease included in the analysis, the proposed treatment algorithm resulted in no further axillary treatment in $24.5 \%$, axillary radiotherapy in $57.3 \%$, and ALND in combination with axillary radiotherapy in $18.2 \%$. As in the present cohort, longer follow-up is needed to prove the long-term oncological safety of omitting ALND with the risk of leaving residual disease behind. Sufficient data have not yet been reported on the long-term outcome of patients with $\mathrm{cN}+$ disease in whom ALND was replaced by less invasive staging procedures.

In previous cohorts that received combination procedures ${ }^{15,18,19}$, sometimes ten or more lymph nodes were retrieved, even though these procedures aim to offer a less invasive alternative to ALND. In the present cohort, only one to three lymph nodes were excised in half of the patients. Furthermore, the median number of excised lymph nodes was the same for patients with and without residual axillary disease. This suggests that the improved detection of residual disease achieved with the combination procedure did not result from removing more lymph nodes.

As ALND was not performed routinely in the present cohort, it was not possible to calculate the FNR and NPV for the combination procedure. Although not all patients underwent ALND, the combination procedure improved the detection rate of residual axillary disease compared with that had the MLN procedure or SLNB been performed as a stand-alone staging procedure. This might because one method covered the failure of the other. An axillary PCR may be predicted based on the MLN, whereas residual axillary disease is predicted based on the $\operatorname{SLN}(\mathrm{s})$ and vice versa. A possible explanation for false-negative $\operatorname{SLN}(s)$ could be residual disease obstructing normal lymphatic drainage or NST altering normal lymphatic drainage. The phenomenon of false-negative SLN(s) has been reported previously by Caudle and colleagues ${ }^{13}$, who noted that the clipped node was not retrieved as a SLN in $23 \%$ of patients in their cohort. A possible explanation for a false-negative MLN could be unsuccessful marking of the most suspicious lymph node, with the marker located in adipose tissue or in non-metastatic lymph nodes, or the existence of differential responses to chemotherapy in the axilla.

The present study is limited by its retrospective design. The combination procedure differed between institutions, although these differences reflect real-world clinical practice. Optimal axillary management after NST in patients with $\mathrm{cN}+$ disease before treatment is currently being investigated in the Alliance $\mathrm{A} 011202^{20}$ and National Surgical Adjuvant Breast and Bowel Project 51/Radiation Therapy Oncology Group $1304^{21}$ trials. These trials will determine optimal management of the axilla based on response to chemotherapy. Until then, the combination procedure for axillary staging 
in patients with $\mathrm{cN}+$ tumours treated with neoadjuvant chemotherapy can be recommended when omission of ALND is considered.

\section{ACKNOWLEDGMENTS}

No preregistration exists for the study reported in this article. J.M.S. received a salary from the Dutch Cancer Society (KWF Kankerbestrijding). 


\section{REFERENCES}

1. Simons JM, van Nijnatten TJA, van der Pol CC, Luiten EJT, Koppert LB, Smidt ML. Diagnostic accuracy of different surgical procedures for axillary staging after neoadjuvant systemic therapy in nodepositive breast cancer: A systematic review and meta-analysis. Ann Surg. 2019;269(3):432-442.

2. Boileau JF, Poirier B, Basik M, et al. Sentinel node biopsy after neoadjuvant chemotherapy in biopsyproven node-positive breast cancer: the SN FNAC study. J Clin Oncol. 2015;33(3):258-264.

3. Boughey JC, Suman VJ, Mittendorf EA, et al. Sentinel lymph node surgery after neoadjuvant chemotherapy in patients with node-positive breast cancer: the ACOSOG Z1071 (Alliance) clinical trial. JAMA. 2013;310(14):1455-1461.

4. Kuehn T, Bauerfeind I, Fehm T, et al. Sentinel-lymph-node biopsy in patients with breast cancer before and after neoadjuvant chemotherapy (SENTINA): a prospective, multicentre cohort study. Lancet Oncol. 2013;14(7):609-618.

5. Donker M, Straver ME, Wesseling J, et al. Marking axillary lymph nodes with radioactive iodine seeds for axillary staging after neoadjuvant systemic treatment in breast cancer patients: the MARI procedure. Ann Surg. 2015;261(2):378-382.

6. van Nijnatten TJ, Schipper RJ, Lobbes MB, Nelemans PJ, Beets-Tan RG, Smidt ML. The diagnostic performance of sentinel lymph node biopsy in pathologically confirmed node positive breast cancer patients after neoadjuvant systemic therapy: A systematic review and meta-analysis. Eur J Surg Oncol. 2015;41(10):1278-1287.

7. Vugts G, Nieuwenhuijzen GA, Maaskant-Braat AJ, Schipper RJ, Smidt ML. Axillary response monitoring after neoadjuvant chemotherapy in breast cancer: Can we avoid the morbidity of axillary treatment? Ann Surg. 2016;263(2):e28-29.

8. Caudle AS, Yang WT, Krishnamurthy S, et al. Improved axillary evaluation following neoadjuvant therapy for patients with node-positive breast cancer using selective evaluation of clipped nodes: Implementation of targeted axillary dissection. J Clin Oncol. 2016;34(10):1072-1078.

9. van Nijnatten TJA, Simons JM, Smidt ML, et al. A novel less-invasive approach for axillary staging after neoadjuvant chemotherapy in patients with axillary node-positive breast cancer by combining radioactive lodine seed localization in the axilla with the sentinel node procedure (RISAS): A Dutch prospective multicenter validation study. Clin Breast Cancer. 2017;17(5):399-402.

10. Hunt KK, Yi M, Mittendorf EA, et al. Sentinel lymph node surgery after neoadjuvant chemotherapy is accurate and reduces the need for axillary dissection in breast cancer patients. Ann Surg. 2009; 250(4):558-566.

11. Curigliano G, Burstein HJ, E PW, et al. De-escalating and escalating treatments for early-stage breast cancer: the St. Gallen International Expert Consensus Conference on the Primary Therapy of Early Breast Cancer 2017. Ann Oncol. 2017;28(8):1700-1712.

12. Siso C, de Torres J, Esgueva-Colmenarejo A, et al. Intraoperative ultrasound-guided excision of axillary clip in patients with node-positive breast cancer treated with neoadjuvant therapy (ILINA Trial) : A new tool to guide the excision of the clipped node after neoadjuvant treatment. Ann Surg Oncol. 2018;25(3):784-791.

13. Caudle AS, Bedrosian I, Milton DR, et al. Use of sentinel lymph node dissection after neoadjuvant chemotherapy in patients with node-positive breast cancer at diagnosis: Practice patterns of American Society of Breast Surgeons Members. Ann Surg Oncol. 2017;24(10):2925-2934.

14. Kim WH, Kim HJ, Jung JH, et al. Ultrasound-guided restaging and localization of axillary lymph nodes after neoadjuvant chemotherapy for guidance of axillary surgery in breast cancer patients: Experience with activated charcoal. Ann Surg Oncol. 2018;25(2):494-500.

15. Park S, Koo JS, Kim GM, et al. Feasibility of charcoal tattooing of cytology-proven metastatic axillary lymph node at diagnosis and sentinel lymph node biopsy after neoadjuvant chemotherapy in breast cancer patients. Cancer Res Treat. 2017.

16. van der Noordaa MEM, van Duijnhoven FH, Straver ME, et al. Major reduction in axillary lymph node dissections after neoadjuvant systemic therapy for node-positive breast cancer by combining PET/CT and the MARI procedure. Ann Surg Oncol. 2018;25(6):1512-1520. 
17. Koolen BB, Donker M, Straver ME, et al. Combined PET-CT and axillary lymph node marking with radioactive iodine seeds (MARI procedure) for tailored axillary treatment in node-positive breast cancer after neoadjuvant therapy. Br J Surg. 2017;104(9):1188-1196.

18. Diego EJ, McAuliffe PF, Soran A, et al. Axillary staging after neoadjuvant chemotherapy for breast cancer: A pilot study combining sentinel lymph node biopsy with radioactive seed localization of pretreatment positive axillary lymph nodes. Ann Surg Oncol. 2016;23(5):1549-1553.

19. Taback B, Jadeja $P, H a$ R. Enhanced axillary evaluation using reflector-guided sentinel lymph node biopsy: A prospective feasibility study and comparison with conventional lymphatic mapping techniques. Clin Breast Cancer. 2018;18(5):e869-e874.

20. NCT01901094. Comparison of axillary lymph node dissection with axillary radiation for patients with node-positive breast cancer treated with chemotherapy. [accessed October 30, 2018]; Available from URL: https://clinicaltrials.gov/ct2/show/NCT01901094.

21. NCT01872975. Standard or comprehensive radiation therapy in treating patients with early-stage breast cancer previously treated with chemotherapy and surgery. [accessed October 30, 2018]; Available from URL: https://clinicaltrials.gov/ct2/show/NCT01872975. 


\section{SUPPLEMENTAL TABLE}

Table S6.1 Pathologic outcome of the combination procedure.

\begin{tabular}{|l|c|c|c|} 
& Outcome ML* & Outcome SLNB* & Outcome combination procedure \\
\hline Scenario A & $\mathrm{ypN}+$ & $\mathrm{ypNO}$ & $\mathrm{ypN+}$ \\
\hline Scenario B & $\mathrm{ypNO}$ & $\mathrm{ypN+}$ & $\mathrm{ypN+}$ \\
\hline Scenario C & $\mathrm{ypN}+$ & $\mathrm{ypN+}$ & $\mathrm{ypN+}$ \\
\hline Scenario D & $\mathrm{ypNO}$ & $\mathrm{ypNO}$ & $\mathrm{ypNO}$ \\
\hline
\end{tabular}

*If the ML and SLN were one and the same node (concordant $\mathrm{ML}$ ), the same pathologic outcome was documented for ML and SLNB. In case additional SLNs were removed, the highest extent of disease was documented as outcome for SLNB (e.g. concordant ML shows ypNO, but additional SLN shows macrometastasis, hence ML outcome is ypNO but SLNB outcome is ypN+). 



\section{PART III}

Prediction of axillary residual disease and prognostic implications 



\section{CHAPTER}

Correlation between pathologic complete response in the breast and absence of axillary lymph node metastases after neoadjuvant systemic therapy

S Samiei

TJA van Nijnatten $L$ de Munck KBMI Keymeulen

JM Simons LFS Kooreman

S Siesling MBI Lobbes ML Smidt 


\section{ABSTRACT}

\section{Objective}

The aim was to investigate whether pathologic complete response $(p C R)$ in the breast is correlated with absence of axillary lymph node metastases at final pathology (ypNO) in patients treated with neoadjuvant systemic therapy (NST) for different breast cancer subtypes.

\section{Background}

Pathologic complete response rates have improved on account of more effective systemic treatment regimens. Promising results in feasibility trials with percutaneous image-guided tissue sampling for the identification of breast PCR after NST raise the question whether breast surgery is a redundant procedure. Thereby, the need for axillary surgery should be reconsidered as well.

\section{Methods}

Patients diagnosed with cT1-3N0-1 breast cancer and treated with NST, followed by surgery between 2010 and 2016, were selected from the Netherlands Cancer Registry. Patients were compared according to the pathologic response of the primary tumor with associated pathologic axillary outcome. Multivariable analysis was performed to determine clinicopathological variables correlated with ypNO.

\section{Results}

A total of 4084 patients were included for analyses, of whom $986(24.1 \%)$ achieved breast pCR. In clinically node negative patients (cNO), 97.7\% (432/442) with breast pCR had ypNO compared with $71.6 \%(882 / 1232)$ without breast pCR $(p<0.001)$. In clinically node positive patients (cN1), 45.0\% (245/544) with breast pCR had ypN0 compared with $9.4 \%(176 / 1866)$ without breast pCR $(p<0.001)$. The odds of ypNO was decreased in case of clinical T3 stage (OR 0.59, 95\% Cl 0.40-0.87), cN1 (OR 0.03, 95\% $\mathrm{Cl} 0.02-0.04$ ) and ER+HER2- subtype (OR $0.30,95 \% \mathrm{Cl} 0.20-0.44)$, and increased in case of breast PCR (OR 4.53, 95\% Cl 3.27-6.28).

\section{Conclusions}

Breast pCR achieved after NST is strongly correlated with ypNO in cNO patients, especially in ER+HER2+, ER-HER2+, and triple negative subtypes. These results provide data to proceed with future clinical trials to investigate if axillary surgery can be safely omitted in these selected patients when image-guided tissue sampling identifies a breast $\mathrm{PCR}$. 


\section{INTRODUCTION}

Over recent years, systemic therapy in the treatment of breast cancer has increasingly been administered in the neoadjuvant setting. The indication of neoadjuvant systemic therapy (NST) has evolved from inoperable and locally advanced breast cancer to early stage breast cancer patients with unfavorable tumor profiles. ${ }^{1}$ NST allows treatment response to be clinically assessed and can lead to the treatment plan being modified in cases of poor response. ${ }^{2}$ It also offers the advantages of downsizing the primary tumor, decreasing the incidence of positive lymph nodes, or even results in complete eradication of cancer, so-called pathologic complete response ( $\mathrm{pCR}$ ) of the breast tumor (hereinafter referred to as breast $\mathrm{PCR}$ ) and/or the axillary lymph nodes (ypN0). ${ }^{3,4}$ As well as these NST advantages, previous studies have reported that $\mathrm{PCR}$ in the breast and axilla is associated with superior survival outcomes. ${ }^{5-7}$

Over the past decade, improvements in the efficacy of chemotherapy and targeted therapies have increased PCR rates. A meta-analysis, performed by Cortazar et al, found that breast pCR was achieved in $22.0 \%$ of patients after NST with higher pCR rates in HER2+ and triple negative breast cancer subtypes. ${ }^{8}$ Concerning axillary lymph nodes, NST can eradicate metastases in clinically node positive patients with a reported axillary $\mathrm{pCR}$ rate of $37.0 \%$. The axillary $\mathrm{pCR}$ rate for HER2+ patients increases with the use of HER2-targeted therapy to between $43 \%$ and $74 \%$. ${ }^{6,7,10,11}$ Patients who achieved axillary pCR were more likely to have breast pCR. ${ }^{6,11,12}$

At present, surgery is the gold standard for determining whether pCR after NST is achieved in breast cancer patients. However, research is increasingly being conducted with the focus on reducing or eliminating breast and/or axillary surgery. Promising results in feasibility trials with percutaneous image-guided biopsy for identifying breast pCR after NST raise the question of whether breast surgery is becoming a redundant procedure in selected group of patients with breast $\mathrm{PCR} .^{13-15}$ Since correlation between breast $\mathrm{PCR}$ and axillary $\mathrm{PCR}$ has been suggested, the need for axillary surgery should be reconsidered in the case of breast $\mathrm{PCR} .{ }^{13,14}$ Evidence for this correlation is limited, however, and not yet studied for different breast cancer subtypes.

Therefore, we aimed to investigate whether breast pCR is correlated with ypNO in patients treated with NST for different breast cancer subtypes. 


\section{METHODS}

In this study, 4084 consecutive patients were included who had all been diagnosed with primary invasive breast cancer in the Netherlands and treated with NST (chemotherapy with or without trastuzumab) between January 2010 and September 2016. To be considered for final analyses, patients needed to be staged as cT1- 3N0-1 breast cancer prior to NST administration. After the completion of NST, all patients underwent standard breast and axillary surgery. Patients were excluded if the sentinel lymph node biopsy (SLNB) had been performed before NST administration. Other exclusion criteria were unknown pathological tumor stage, distant metastases at primary breast cancer diagnosis or within 91 days after surgery, unknown breast cancer subtype, neoadjuvant endocrine therapy, or unknown number of lymph node metastases at final pathology (Figure 7.1).

Figure 7.1 Flow diagram of patient inclusion.

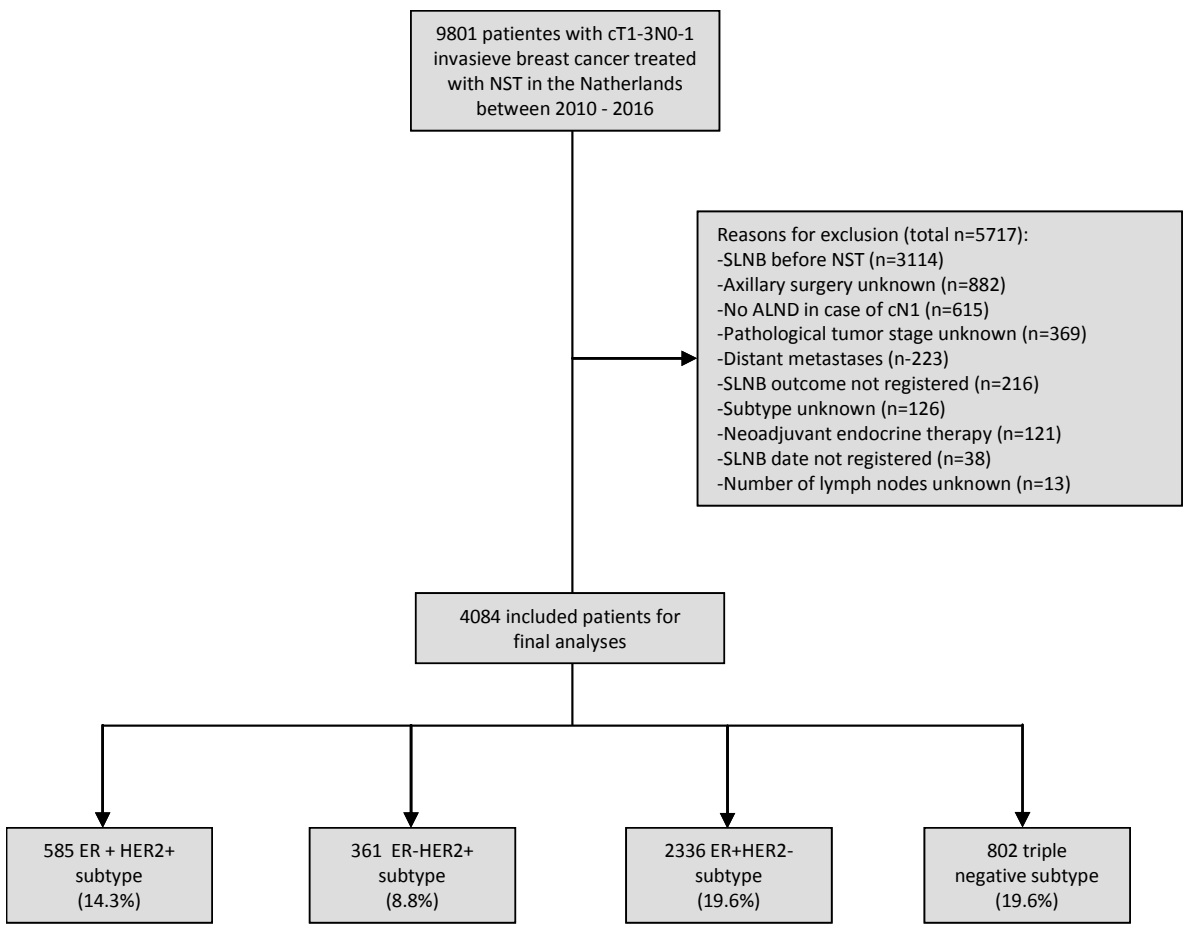

NST, neoadjuvant systemic therapy; SLNB, sentinel lymph node biopsy; ALND, axillary lymph node dissection; cN1, clinically node positive; ER, estrogen receptor; HER2, human epidermal growth factor receptor 2; Triple negative, negative for ER, PR, and HER2 
The axillary nodal status was determined before NST administration by axillary ultrasound. If axillary ultrasound showed no suspicious lymph nodes, patients were defined as clinically node negative (cNO). If suspicious lymph nodes were confirmed with additional fine-needle aspiration cytology (FNAC) or core needle biopsy, patients were defined as clinically node positive ( $\mathrm{CN} 1)$. If the patient was defined as cNO before NST administration, the patient underwent SLNB after NST. If the patient was classified as cN1 before NST administration, the patient underwent axillary lymph node dissection (ALND) after NST.

The 2008 and 2012 Dutch national guidelines were applied during the 2010 to 2016 study period. ${ }^{16,17}$ These guidelines recommended systemic therapy consisting of the following chemotherapeutic regimens: 6 cycles of TAC (Taxotere, Adriamycin, Cyclofosfamide), or 3 cycles of FEC (Fluorouracil (5FU), Epirubicin, Cyclophosphamide), or 4 cycles of AC (Adriamycine, Cyclophosphamide) followed by 12 cycles of paclitaxel or 4 cycles of docetaxel. In the case of HER2+ breast cancer, trastuzumab was recommended as the targeted therapy in addition to chemotherapy and continued until 1 year after the start. In the time frame 2010 to 2016 no HER2-targeted therapy was advised in addition to trastuzumab.

HER2 status was evaluated with immunohistochemistry (IHC). The IHC score of 0 or $1+$ was considered negative $(<10 \%$ of the tumor cells are stained, or $>10 \%$ of the tumor cells are stained, with no circumferential staining and weak color intensity). In case of a $2+$ IHC score ( $>10 \%$ circumferential membrane staining with moderate intensity), fluorescence in situ hybridization (FISH) was mandatory in addition to IHC and the result of FISH overruled. The IHC score of $3+$ was considered positive $(>30 \%$ of cells with strong intensity circumferential membrane staining).

Data were obtained from the Netherlands Cancer Registry (NCR) after this study had been approved by the Privacy Review Board of NCR, managed by the Netherlands Comprehensive Cancer Organisation (IKNL). On site trained registrars from the NCR extract data from patients' medical records after notification. Data were collected on age, tumor histology, receptor status, surgical procedures, systemic therapy, radiation therapy, clinical TNM stage, and pathology results, including stage after NST (ypTNM), tumor grade and number of axillary lymph nodes with and without metastases. Breast pCR was defined as the absence of both invasive and in situ breast cancer, with ypNO being defined as the absence of both macro- and micrometastases in the axillary lymph nodes. ${ }^{18}$ Isolated tumor cells were considered as ypNo. ${ }^{18}$

Statistical analyses were performed by using Statistical Package for the Social Sciences software (SPSS, version 24, IBM, Armonk, NY). Patients were subdivided into breast pCR or without breast $\mathrm{pCR}$ for the following breast cancer subtypes: ER 
positive(+)HER2+, ER negative-HER2+, ER+HER2-, and triple negative. For each subtype, the number of axillary lymph nodes with and without metastases at final pathology was reported. The $\chi^{2}$ test and Fisher exact test were used to compare patients with breast $\mathrm{pCR}$ and without breast $\mathrm{pCR}$. Univariable logistic regression analysis was conducted to determine the association between relevant clinicopathological variables and ypNO. Multivariable analysis was performed to identify the independent clinicopathological variables correlated with ypNO. Odds ratios (ORs) with $95 \%$ confidence intervals (Cls) were calculated. Two-sided $p$ values of $<0.05$ were considered statistically significant.

\section{RESULTS}

Of the 4084 patients (median age, 49 yrs; range, 18-83 yrs) included for analyses, 585 patients had ER+HER2+, 361 ER- HER2+, 2336 ER+HER2- and 802 had triple negative breast cancer subtype. A total of 1674 (41.0\%) patients were cNO and 2410 (59.0\%) were cN1. Breast conserving surgery was performed in 1835 (44.9\%) patients and 2249 (55.1\%) underwent mastectomy. SLNB was performed in 1483 (36.3\%) patients and 2601 (63.7\%) underwent ALND (Table 7.1).

Overall, 986 out of 4084 patients (24.1\%) achieved breast pCR. Of the patients with breast pCR, 68.7\% (677 of 986) had ypNO compared with $34.2 \%$ (1058 of 3098) without breast pCR $(p<0.001)$. In the case of cN0, 97.7\% $(432 / 442)$ with breast pCR had ypNO compared with $71.6 \%(882 / 1232)$ without breast $\mathrm{pCR}(\mathrm{p}<0.001)$. In the case of cN1, $45.0 \%(245 / 544)$ with breast pCR had ypNO compared to $9.4 \%(176 / 1866)$ without breast $\mathrm{pCR}(\mathrm{p}<0.001)$ (Table 7.2).

In univariable analysis, clinicopathological variables associated with lower odds of ypNO were age 35 to 50 years (OR $0.70,95 \% \mathrm{Cl} 0.54-0.90, \mathrm{p}<0.006$ ), age 50 to 75 years (OR 0.60, 95\% Cl 0.47-0.79, p<0.001), clinical T3 stage (OR 0.51, 95\% Cl 0.41-0.62, $\mathrm{p}<0.001$ ), cN1 stage (OR 0.06, 95\% Cl 0.05-0.07, p<0.001), ER+HER2- subtype (OR 0.32, $95 \% \mathrm{Cl} 0.27-0.39, \mathrm{p}<0.001$ ), and triple negative subtype (OR $0.31,95 \% \mathrm{Cl} 0.26-0.37$, $\mathrm{p}<0.001$ ). Tumor grade 3 (OR 1.67, 95\% Cl 1.28-2.19, p=0.045) and breast $p C R$ (OR $4.22,95 \% \mathrm{Cl} 3.62-4.93, \mathrm{p}<0.001$ ) were associated with higher odds of ypNO. In multivariable analysis after correcting for confounders, the odds of ypNO was decreased in the case of clinical T3 stage (OR 0.59, 95\% Cl 0.40-0.87, p<0.007), cN1 (OR $0.03,95 \% \mathrm{Cl} 0.02-0.04, \mathrm{p}<0.001$ ) and ER+HER2- subtype (OR $0.30,95 \% \mathrm{Cl} 0.20-$ $0.44, p<0.001$ ), and increased in the case of breast pCR (OR $4.53,95 \% \mathrm{Cl} 3.27-6.28$, $\mathrm{p}<0.001$ ) (Table 7.3). 
Table 7.1 Overview of patient, tumor and treatment characteristics.

\begin{tabular}{|c|c|c|c|c|c|}
\hline & $\begin{array}{c}\text { Total } \\
(n=4084)\end{array}$ & $\begin{array}{c}\text { ER+HER2+ } \\
(n=585)\end{array}$ & $\begin{array}{c}\text { ER-HER2+ } \\
(n=361)\end{array}$ & $\begin{array}{l}\text { ER+HER2- } \\
(n=2336)\end{array}$ & $\begin{array}{c}\text { Triple negative } \\
\quad(n=802)\end{array}$ \\
\hline Median age (years; range) & $49(18-83)$ & $48(18-75)$ & $51(23-81)$ & $50(24-76)$ & $49(24-83)$ \\
\hline \multicolumn{6}{|l|}{ Clinical stage (\%) } \\
\hline T1N0 & $294(7.2)$ & $59(10.1)$ & $15(4.2)$ & $159(6.4)$ & $61(7.6)$ \\
\hline T1N1 & $355(8.7)$ & $42(7.2)$ & $29(8.0)$ & $210(9.8)$ & $74(9.2)$ \\
\hline T2NO & $1057(25.9)$ & $172(29.4)$ & $88(24.4)$ & $557(20.2)$ & $240(30.0)$ \\
\hline $\mathrm{T} 2 \mathrm{~N} 1$ & $1354(33.2)$ & $181(30.9)$ & $120(33.2)$ & $773(36.2)$ & $280(34.9)$ \\
\hline T3NO & $323(7.9)$ & $46(7.9)$ & $25(6.9)$ & $216(7.6)$ & $36(4.5)$ \\
\hline T3N1 & $701(17.1)$ & $85(14.5)$ & $84(23.3)$ & $421(19.8)$ & $111(13.8)$ \\
\hline \multicolumn{6}{|l|}{ Tumor histology (\%) } \\
\hline Ductal & $3242(79.4)$ & $510(87.2)$ & $318(88.1)$ & $1725(73.8)$ & 689 (85.9) \\
\hline Lobular & 439 (10.7) & $29(5.0)$ & $8(2.2)$ & $388(16.6)$ & $14(1.7)$ \\
\hline Mixed ductal and lobular & $103(2.5)$ & $11(1.9)$ & $2(0.6)$ & $88(3.8)$ & $2(0.2)$ \\
\hline Other $^{a}$ & $300(7.4)$ & $35(5.9)$ & $33(9.1)$ & $135(5.8)$ & $97(12.2)$ \\
\hline \multicolumn{6}{|l|}{ Tumor grade (\%) } \\
\hline 1 & $293(7.1)$ & $25(4.3)$ & $10(2.8)$ & $253(10.8)$ & $5(0.6)$ \\
\hline 2 & $1090(26.7)$ & $170(29.1)$ & $60(16.6)$ & $764(32.7)$ & $96(12.0)$ \\
\hline 3 & $864(21.2)$ & $126(21.5)$ & $105(29.1)$ & $258(11.0)$ & $375(46.8)$ \\
\hline Unknown & $1837(45.0)$ & $264(45.1)$ & $186(51.5)$ & $1061(45.5)$ & $326(40.6)$ \\
\hline \multicolumn{6}{|l|}{ Breast surgery (\%) } \\
\hline Breast-conserving surgery & 1835 (44.9) & $304(52.0)$ & $169(46.8)$ & $967(41.4)$ & 395 (49.3) \\
\hline Mastectomy & $2249(55.1)$ & $281(48.0)$ & $192(53.2)$ & $1369(58.6)$ & $407(50.7)$ \\
\hline \multicolumn{6}{|l|}{ Axillary surgery (\%) } \\
\hline SLNB & $1483(36.3)$ & 257 (43.9) & $123(34.1)$ & 791 (33.9) & 312 (38.9) \\
\hline ALND & $2601(63.7)$ & $328(56.1)$ & $238(65.9)$ & $1545(66.1)$ & $490(61.1)$ \\
\hline Breast pCR (\%) & $986(24.1)$ & $236(40.3)$ & $247(68.4)$ & $208(8.9)$ & $295(36.8)$ \\
\hline
\end{tabular}

a Includes adenocarcinoma not further defined, mucinous adenocarcinoma, medullary carcinoma, metaplastic carcinoma among other things. ER, estrogen receptor; HER2, human epidermal growth factor receptor 2; Triple negative, negative for ER, PR and HER2; SLNB, sentinel lymph node biopsy; ALND, axillary lymph node dissection; $\mathrm{PCR}$, pathologic complete response 
Table 7.2 Overview of number of lymph node metastases for each breast cancer subtype differentiated between breast PCR and without breast PCR after NST.

\begin{tabular}{|c|c|c|c|c|c|c|}
\hline \multirow{2}{*}{$\begin{array}{l}\text { Breast pCR } \\
(n=986)\end{array}$} & & \multicolumn{5}{|c|}{ Number of Lymph Node Metastases on Final Pathology (\%) } \\
\hline & & 0 & 1 & 2 & 3 & $\geq 4$ \\
\hline \multirow{6}{*}{$\begin{array}{l}\text { ER+HER2+ } \\
(n=236)\end{array}$} & cT1N0 & $29(100)$ & 0 & 0 & 0 & 0 \\
\hline & cT2NO & 73 (99) & $1(1)$ & 0 & 0 & 0 \\
\hline & cT3NO & $20(95)$ & $1(5)$ & 0 & 0 & 0 \\
\hline & cT1N1 & $9(60)$ & $6(40)$ & 0 & 0 & 0 \\
\hline & cT2N1 & $33(53)$ & $26(42)$ & 0 & $2(3)$ & $1(2)$ \\
\hline & cT3N1 & $16(46)$ & $17(48)$ & 0 & $1(3)$ & $1(3)$ \\
\hline \multirow{6}{*}{$\begin{array}{l}\text { ER-HER2+ } \\
(n=247)\end{array}$} & cT1N0 & $13(100)$ & 0 & 0 & 0 & 0 \\
\hline & cT2NO & $72(100)$ & 0 & 0 & 0 & 0 \\
\hline & cT3NO & $12(100)$ & 0 & 0 & 0 & 0 \\
\hline & cT1N1 & $12(48)$ & $13(52)$ & 0 & 0 & 0 \\
\hline & cT2N1 & $33(43)$ & $42(55)$ & $1(1)$ & $1(1)$ & 0 \\
\hline & cT3N1 & $25(52)$ & $23(48)$ & 0 & 0 & 0 \\
\hline \multirow{6}{*}{$\begin{array}{l}\text { ER+HER2- } \\
(n=208)\end{array}$} & cT1N0 & $27(94)$ & $1(3)$ & $1(3)$ & 0 & 0 \\
\hline & cT2NO & $44(94)$ & $3(6)$ & 0 & 0 & 0 \\
\hline & cT3NO & $12(92)$ & $1(8)$ & 0 & 0 & 0 \\
\hline & cT1N1 & $4(17)$ & 12 (49) & $4(17)$ & 0 & $4(17)$ \\
\hline & cT2N1 & $25(35)$ & $33(46)$ & $6(8)$ & $2(3)$ & $6(8)$ \\
\hline & cT3N1 & 9 (39) & $10(43)$ & 0 & $2(9)$ & $2(9)$ \\
\hline \multirow{6}{*}{$\begin{array}{l}\text { Triple } \\
\text { negative } \\
(n=295)\end{array}$} & cT1N0 & $29(97)$ & 0 & $1(3)$ & 0 & 0 \\
\hline & cT2NO & $99(100)$ & 0 & 0 & 0 & 0 \\
\hline & cT3NO & $2(67)$ & $1(33)$ & 0 & 0 & 0 \\
\hline & cT1N1 & $16(49)$ & $15(45)$ & $1(3)$ & 0 & $1(3)$ \\
\hline & cT2N1 & 47 (47) & 45 (44) & $6(6)$ & $1(1)$ & $2(2)$ \\
\hline & cT3N1 & $16(55)$ & $10(35)$ & $2(7)$ & 0 & $1(3)$ \\
\hline
\end{tabular}

\begin{tabular}{ll|c|c|c|c|c}
\multirow{2}{*}{$\begin{array}{l}\text { No Breast pCR } \\
\text { (n=3098) }\end{array}$} & \multicolumn{6}{c}{ Number of Lymph Node Metastases on Final Pathology (\%) } \\
\cline { 3 - 7 } & & $\mathbf{0}$ & $\mathbf{1}$ & $\mathbf{2}$ & $\mathbf{3}$ & $\geq 4$ \\
\hline \hline & cT1N0 & $25(83)$ & $5(17)$ & 0 & 0 & 0 \\
\multirow{2}{*}{ ER+HER2+ } & cT2N0 & $87(89)$ & $8(8)$ & $2(2)$ & 0 & $1(1)$ \\
$(n=349)$ & cT3N0 & $18(72)$ & $5(20)$ & $1(4)$ & 0 & $1(4)$ \\
& cT2N1 & $9(33)$ & $10(37)$ & $2(7)$ & $1(4)$ & $5(19)$ \\
& cT3N1 & $15(13)$ & $63(53)$ & $10(8)$ & $7(6)$ & $24(20)$ \\
& cT1N0 & $15(30)$ & $13(26)$ & $4(8)$ & $3(6)$ & $15(30)$ \\
\hline & cT2N0 & $2(100)$ & 0 & 0 & 0 & 0 \\
ER-HER2+ & cT3N0 & $14(88)$ & $2(12)$ & 0 & 0 & 0 \\
$(n=114)$ & cT1N1 & $12(92)$ & 0 & $1(8)$ & 0 & 0 \\
& cT2N1 & $2(50)$ & 0 & 0 & $2(50)$ & 0 \\
& cT3N1 & $14(33)$ & $13(30)$ & $6(14)$ & $4(9)$ & $6(14)$ \\
\hline
\end{tabular}


Table 7.2 (continued)

\begin{tabular}{|c|c|c|c|c|c|c|}
\hline \multirow{2}{*}{$\begin{array}{l}\text { No Breast pCR } \\
(n=3098)\end{array}$} & & \multicolumn{5}{|c|}{ Number of Lymph Node Metastases on Final Pathology (\%) } \\
\hline & & 0 & 1 & 2 & 3 & $\geq 4$ \\
\hline \multirow{6}{*}{$\begin{array}{l}\text { ER+HER2- } \\
(n=2128)\end{array}$} & cT1N0 & $97(75)$ & $24(18)$ & $5(4)$ & 0 & $4(3)$ \\
\hline & cT2NO & $341(67)$ & $124(24)$ & $23(4)$ & $10(2)$ & $12(3)$ \\
\hline & cT3NO & $107(53)$ & $58(29)$ & $11(5)$ & $4(2)$ & $23(11)$ \\
\hline & cT1N1 & $7(4)$ & $63(34)$ & $34(18)$ & $19(10)$ & $63(34)$ \\
\hline & cT2N1 & $36(5)$ & $217(31)$ & $111(16)$ & $88(13)$ & 249 (35) \\
\hline & cT3N1 & $22(6)$ & $72(18)$ & $45(11)$ & $46(12)$ & $213(53)$ \\
\hline \multirow{6}{*}{$\begin{array}{l}\text { Triple negative } \\
(n=507)\end{array}$} & cT1N0 & $27(87)$ & $4(13)$ & 0 & 0 & 0 \\
\hline & cT2NO & 126 (89) & $12(8)$ & $2(2)$ & $1(1)$ & 0 \\
\hline & cT3NO & $26(79)$ & $4(12)$ & $2(6)$ & 0 & $1(3)$ \\
\hline & cT1N1 & $5(12)$ & $15(37)$ & $4(10)$ & $5(12)$ & 12 (29) \\
\hline & cT2N1 & $35(20)$ & $64(36)$ & $20(11)$ & $15(8)$ & 45 (25) \\
\hline & cT3N1 & 7 (9) & $18(22)$ & $10(12)$ & $7(9)$ & $40(52)$ \\
\hline
\end{tabular}

pCR, pathological complete response; NST, neoadjuvant systemic therapy; ER, estrogen receptor; HER2, human epidermal growth factor receptor 2; Triple negative, negative for ER, PR, and HER2.

\section{ER+HER2+ subtype}

Trastuzumab in neoadjuvant setting was administered in 531 out of 585 (90.8\%) ER+HER2+ patients. In this subtype, 236 out of 585 (40.3\%) patients achieved breast pCR. In all ER+HER2+ patients with breast pCR, 76.3\% (180 of 236) had ypNO compared to $48.4 \%$ (169 of 349 ) without breast $p C R(p<0.001)$. In the case of $c N 0$ with breast pCR, only 2 out of 124 patients (1.6\%) had 1 axillary lymph node metastasis at final pathology, compared to $15.0 \%$ (23 of 153) without breast pCR $(p<0.001)$. In the case of $\mathrm{cN} 1$ with breast $\mathrm{pCR}, 51.8 \%$ (58 of 112) of the patients had ypN0 compared to $19.9 \%$ (39 of 196) without breast pCR ( $<<0.001)$ (Table 7.4).

\section{ER-HER2+ subtype}

In the ER-HER2+ subtype, trastuzumab in neoadjuvant setting was administered in 336 out of 361 (93.1\%) patients. In this subtype, 247 out of 361 (68.4\%) patients achieved breast pCR. In patients with breast pCR, 67.6\% (167 of 247) had ypNO compared to $46.5 \%$ (53 of 114 ) without breast $p C R(p<0.001)$. All cNO patients with breast $p C R$ $(n=97)$ had ypNO compared to $90.3 \%$ ( 28 of 31$)$ without breast $p C R(p=0.013)$. In the case of $\mathrm{cN} 1$ with breast $\mathrm{pCR}, 46.7 \%$ (70 of 150) of the patients had ypNO compared to $30.1 \%$ ( 25 of 83 ) without breast $\mathrm{pCR}(\mathrm{p}=0.014)$. 
Table 7.3 Univariable and multivariable analyses for clinicopathological variables and the outcome ypNO after NST.

\begin{tabular}{|c|c|c|c|c|c|c|}
\hline & \multicolumn{3}{|c|}{ Univariable analysis } & \multicolumn{3}{|c|}{ Multivariable analysis } \\
\hline & OR & $95 \% \mathrm{Cl}$ & p-value & OR & $95 \% \mathrm{Cl}$ & p-value \\
\hline $\begin{array}{l}\text { Age } \\
<35 \text { years } \\
35-50 \text { years } \\
50-75 \text { years } \\
>75 \text { years }\end{array}$ & $\begin{array}{c}1 \text { [reference] } \\
0.70 \\
0.61 \\
0.60\end{array}$ & $\begin{array}{l}0.54-0.90 \\
0.47-0.79 \\
0.21-1.74\end{array}$ & $\begin{array}{c}p<0.006 \\
p<0.001 \\
p=0.35\end{array}$ & $\begin{array}{c}1 \text { [reference] } \\
0.91 \\
0.90 \\
1.55\end{array}$ & $\begin{array}{c}0.55-1.50 \\
0.55-1.48 \\
0.20-12.15\end{array}$ & $\begin{array}{l}p=0.72 \\
p=0.68 \\
p=0.68\end{array}$ \\
\hline $\begin{array}{l}\text { Clinical tumor } \\
\text { stage } \\
\text { T1 } \\
\text { T2 } \\
\text { T3 } \\
\end{array}$ & $\begin{array}{c}1 \text { [reference] } \\
0.89 \\
0.51\end{array}$ & $\begin{array}{l}0.75-1.06 \\
0.41-0.62\end{array}$ & $\begin{array}{c}p=0.20 \\
p<0.001\end{array}$ & $\begin{array}{c}1 \text { [reference] } \\
0.89 \\
0.59\end{array}$ & $\begin{array}{l}0.64-1.24 \\
0.40-0.87\end{array}$ & $\begin{array}{c}p=0.49 \\
p=0.007\end{array}$ \\
\hline $\begin{array}{l}\text { Clinical nodal } \\
\text { stage } \\
\text { NO } \\
\text { N1 }\end{array}$ & $\begin{array}{c}1 \text { [reference] } \\
0.06\end{array}$ & $0.05-0.07$ & $p<0.001$ & $\begin{array}{c}1 \text { [reference] } \\
0.03\end{array}$ & $0.02-0.04$ & $p<0.001$ \\
\hline $\begin{array}{l}\text { Tumor histology } \\
\text { Ductal } \\
\text { Lobular } \\
\text { Other } \\
\end{array}$ & $\begin{array}{c}1 \text { [reference] } \\
0.84 \\
0.98 \\
\end{array}$ & \begin{tabular}{|l|}
$0.68-1.03$ \\
$0.79-1.21$ \\
\end{tabular} & $\begin{array}{c}p=0.089 \\
p=0.83\end{array}$ & $\begin{array}{c}1 \text { [reference] } \\
0.93 \\
0.89 \\
\end{array}$ & $\begin{array}{l}0.63-1.37 \\
0.59-1.36 \\
\end{array}$ & $\begin{array}{l}p=0.70 \\
p=0.60\end{array}$ \\
\hline $\begin{array}{c}\text { Tumor grade } \\
1 \\
2 \\
3 \\
\end{array}$ & $\begin{array}{c}1 \text { [reference] } \\
1.09 \\
1.67\end{array}$ & \begin{tabular}{|l|}
$0.84-1.41$ \\
$1.28-2.19$ \\
\end{tabular} & $\begin{array}{c}p=0.53 \\
p<0.001\end{array}$ & $\begin{array}{c}1 \text { [reference] } \\
1.01 \\
1.12 \\
\end{array}$ & $\begin{array}{l}0.71-1.45 \\
0.74-1.69\end{array}$ & $\begin{array}{l}p=0.95 \\
p=0.59\end{array}$ \\
\hline $\begin{array}{c}\text { Tumor subtype } \\
\text { ER+HER2+ } \\
\text { ER-HER2+ } \\
\text { ER+HER2- } \\
\text { Triple negative } \\
\end{array}$ & $\begin{array}{c}1 \text { [reference] } \\
1.06 \\
0.31 \\
0.80 \\
\end{array}$ & $\begin{array}{l}0.81-1.38 \\
0.26-0.37 \\
0.65-0.96 \\
\end{array}$ & $\begin{array}{l}p=0.70 \\
p<0.001 \\
p=0.045\end{array}$ & $\begin{array}{c}1 \text { [reference] } \\
1.16 \\
0.30 \\
0.95\end{array}$ & $\begin{array}{l}0.68-1.98 \\
0.20-0.44 \\
0.62-1.45\end{array}$ & $\begin{array}{c}p=0.60 \\
p<0.001 \\
p=0.81\end{array}$ \\
\hline $\begin{array}{l}\text { Breast pCR } \\
\text { No } \\
\text { Yes }\end{array}$ & $\begin{array}{c}1 \text { [reference] } \\
4.22\end{array}$ & $3.62-4.93$ & $\mathrm{p}<0.001$ & $\begin{array}{c}1 \text { [reference] } \\
4.53\end{array}$ & $3.27-6.28$ & $p<0.001$ \\
\hline
\end{tabular}

$\mathrm{pCR}$, pathologic complete response; NST, neoadjuvant systemic therapy; OR, odds ratio; $\mathrm{Cl}$, confidence interval; ER, estrogen receptor; HER2, human epidermal growth factor receptor 2; Triple negative, negative for ER, PR, and HER2.

\section{ER+HER2- subtype}

In the ER+HER2- subtype, 208 out of 2336 (8.9\%) patients achieved breast pCR of whom $58.2 \%$ (121 of 208) had ypNO compared to $28.7 \%$ (610 of 2128) without breast pCR ( $p<0.001)$. In cNO patients with breast pCR, 6 out of 89 patients $(6.7 \%)$ had 1 or 2 axillary lymph node metastases at final pathology, compared to $35.3 \%$ (298 of 843) without breast $p C R(p<0.001)$. In $c N 1$ patients with breast $p C R, 31.9 \%$ (38 of 119) of the patients had ypNO compared to $5.1 \%$ (65 of 1285) without breast $p C R(p<0.001)$. 
Table 7.4 Overview of ypNO in the case of breast pCR and without breast pCR after NST for the different breast cancer subtypes.

\begin{tabular}{l||c|c|c} 
& Breast $p$ CR and ypN0 & No breast $p$ CR and ypN0 & $p^{-v a l u e}{ }^{a}$ \\
\hline \hline cT1-3N0 ER+HER2+ $(n=277)$ & $98.4(122 / 124)$ & $85.0(130 / 153)$ & $p<0.001$ \\
\hline cT1-3N1 ER+HER2+ $(n=308)$ & $51.8(58 / 112)$ & $19.9(39 / 196)$ & $p<0.001$ \\
\hline cT1-3N0 ER-HER2+ $(n=128)$ & $100(97 / 97)$ & $90.3(28 / 31)$ & $p=0.013$ \\
\hline cT1-3N1 ER-HER2+ $(n=233)$ & $46.7(70 / 150)$ & $30.1(25 / 83)$ & $p=0.014$ \\
\hline cT1-3N0 ER+HER2- $(n=932)$ & $93.3(83 / 89)$ & $64.7(545 / 843)$ & $p<0.001$ \\
\hline cT1-3N1 ER+HER2- $(n=1404)$ & $31.9(38 / 119)$ & $5.1(65 / 1285)$ & $p<0.001$ \\
\hline cT1-3N0 triple negative $(n=337)$ & $98.5(130 / 132)$ & $87.3(179 / 205)$ & $p<0.001$ \\
\hline cT1-3N1 triple negative $(n=465)$ & $48.5(79 / 163)$ & $15.6(47 / 302)$ & $p<0.001$ \\
\hline
\end{tabular}

pCR, pathologic complete response; NST, neoadjuvant systemic therapy; ER, estrogen receptor; HER2, human epidermal growth factor receptor 2; Triple negative, negative for ER, PR, and HER2. ${ }^{\mathrm{a}} \boldsymbol{\chi}^{2}$ test and Fisher's exact test between patients with breast $\mathrm{pCR}$ and ypNO versus patients without breast $\mathrm{pCR}$ and ypNO. Data are presented as percentages with the numbers in parentheses.

\section{Triple negative subtype}

In the triple negative subtype, 295 out of 802 (36.8\%) patients achieved breast pCR of whom $70.8 \%$ (209 of 295) had ypN0 compared to $44.6 \%$ (226 of 507) without breast pCR $(p<0.001)$. In the case of cNO with breast pCR, only 2 out of 132 patients $(1.5 \%)$ had 1 or 2 axillary lymph node metastases at final pathology, compared to $12.7 \%$ (26 of 205) without breast pCR ( $p<0.001)$. In cN1 patients with breast $p C R, 48.5 \%$ (79 of 163) of the patients had ypN0 compared to $15.6 \%$ (47 of 302) without breast pCR $(p<0.001)$.

\section{DISCUSSION}

In this study, we have reported on a large cohort of cT1-3N0-1 breast cancer patients who were treated with NST and showed an overall breast pCR rate of $24.1 \%$. We found that breast PCR achieved after NST is positively correlated with ypNO. Further, the findings showed that in the case of breast pCR, $97.7 \%$ of cNO patients had ypNO and in the case of $\mathrm{cN} 145.0 \%$ converted to ypNO. Of all breast cancer subtypes, only the patients with ER+HER2- subtype were less likely to have ypNO.

Recently, Tadros et al ${ }^{14}$ have demonstrated that breast $\mathrm{PCR}$ is strongly correlated with axillary nodal status after NST. They showed a total breast pCR rate of $36.6 \%$ (193 of $527)$ with a slightly higher rate of breast $\mathrm{PCR}$ in the triple negative group $(37.5 \%)$ compared to the HER2+ group (35.7\%). In contrast to the Tadros et al study, patients with clinical T3 stage and ER+HER2- subtype were also included in the present study which can explain the lower total breast $\mathrm{PCR}$ rate here. We found that in the case of 
ER+HER2- breast cancer, $\mathrm{pCR}$ rates of the breast and axilla were lower compared to the other breast cancer subtypes. For this subtype, the most important systemic therapy (ie, hormonal therapy) generally follows in the adjuvant setting. Previous studies had reported breast and axillary $\mathrm{PCR}$ rates for the ER+HER2- subtype ranging from $7.5 \%$ to $16.5 \% .{ }^{4,7,8}$ We have shown similar breast pCR rates, but higher ypNO rates due to the included number of cNO patients. In the Tadros et al study, all 527 cT1-2NO patients with HER2+ and triple negative breast cancer who achieved breast pCR after NST were found to have ypNO. ${ }^{14}$ We have confirmed this strong correlation here between breast pCR and ypNO in cNO patients for ER+HER2+, ER-HER2+, and triple negative breast cancer subtypes.

Due to the presence of more effective treatment regimens, $\mathrm{PCR}$ rates in the breast and axilla have improved dramatically over the past decade. ${ }^{19}$ Ongoing trials are currently evaluating the accuracy of image-guided minimally invasive techniques for predicting breast $P C R$ in order to potentially omit surgery. ${ }^{20,21}$ In the MICRA trial, biopsies of the original tumor bed are obtained after NST and prior to surgery in all patients with complete or partial radiologic response evaluated by MRI. ${ }^{20}$ Preliminary results show that ultrasound-guided biopsies identify breast pCR successfully in $91.4 \%$ (43 out of 47 ) of the patients after NST. In a study by Kuerer et al, ${ }^{22}$ FNAC and imageguided vacuum-assisted biopsy of the tumor bed accurately identified breast PCR after chemotherapy in $98.0 \%$ (38 out of 40 ) of the patients with a false negative rate (FNR) of $5.0 \%$. The Kuerer et al. findings have resulted in a prospective clinical trial evaluating omission of breast surgery after NST in patients with breast pCR confirmed by image-guided tissue sampling. ${ }^{21}$

Identifying breast pCR appears important for guiding axillary treatment given the previously shown correlation between breast and axillary $\mathrm{PCR} .{ }^{13,14}$ If breast $\mathrm{PCR}$ after NST can be identified prior to surgery, resulting in the complete omission of breast surgery, how should the axillary lymph nodes be handled in such patients? In recent years the focus has been on minimizing axillary surgery in an aim to reduce surgical morbidity. In this study, we observed that $97.7 \%$ of cNO breast cancer patients who achieved breast pCR had ypNO. This implies that the risk of missing patients with axillary lymph node metastases in these selected patients is highly unlikely. Therefore, these patients should proceed to clinical trials to evaluate the safety of omission of axillary surgery when breast pCR after NST is identified by image-guided tissue sampling. ${ }^{21}$ The requirement to proceed in these clinical trials is the determination of the axillary nodal status before NST administration by axillary ultrasound with or without biopsy.

In our study, only $45.0 \%$ of all cN1 patients who achieved breast pCR also had ypN0. Consequently, these cN1 patients remain at risk of having axillary lymph node 
metastases at final pathology, irrespective of breast $\mathrm{pCR}$, and omission of axillary surgery would therefore be inappropriate. The performance of imaging techniques for assessing residual disease in the axillary lymph nodes after NST remains inaccurate. ${ }^{23,24}$ As for these cN1 patients, minimally invasive surgical methods for accurately predicting the axillary status are currently under investigation. These minimally invasive techniques aim to identify ypNO after NST resulting in less ALND and thereby preventing the associated morbidity. Caudle et al showed in a retrospective study of 85 clinically node positive patients that SLNB in combination with selective removal of metastatic marked nodes for predicting ypNO after NST has a FNR of $2.0 \% .^{25}$ The Dutch RISAS trial is a currently ongoing prospective multicenter study to validate this combined procedure of SLNB and MARI (marking the axillary lymph nodes with radioactive seeds) for identifying ypNO after NST in clinically node positive patients. $^{26}$

\section{Limitations}

This study included patients from all institutions in the Netherlands and thereby an advantage is generalizability of the results. However, our study has certain limitations. Due to the retrospective nature of the data, there can be no guarantee that all patients completed NST and, therefore, were treated sufficiently, since this may have contributed to not obtaining breast and/or axillary pCR. Additionally, the number of excluded patients as a consequence of missing data could have affected our results, such as unknown pathological tumor stage and breast cancer subtype.

\section{Conclusions}

These results indicate that cNO patients who achieve breast pCR after NST are highly likely to achieve ypNO, especially in ER+HER2+, ER-HER2+, and triple negative breast cancer subtypes. Besides guiding omission of breast surgery, identifying breast $\mathrm{PCR}$ may guide de-escalating axillary treatment with the potential to omit axillary surgery in selected patients. Future clinical trials should investigate if omission of axillary surgery in these selected patients is safe when image-guided tissue sampling identifies breast pCR after NST. 


\section{REFERENCES}

1. Mamounas EP. Impact of neoadjuvant chemotherapy on locoregional surgical treatment of breast cancer. Ann Surg Oncol. 2015;22(5):1425-1433.

2. Schott $A F$, Roubidoux MA, Helvie $M A$, et al. Clinical and radiologic assessments to predict breast cancer pathologic complete response to neoadjuvant chemotherapy. Breast Cancer Res Treat. 2005;92(3):231-238.

3. Fisher B, Brown A, Mamounas E, et al. Effect of preoperative chemotherapy on local-regional disease in women with operable breast cancer: Findings from National Surgical Adjuvant Breast and Bowel Project B-18. Journal of Clinical Oncology. 1997;15(7):2483-2493.

4. Houssami N, Macaskill P, von Minckwitz G, Marinovich ML, Mamounas E. Meta-analysis of the association of breast cancer subtype and pathologic complete response to neoadjuvant chemotherapy. Eur J Cancer. 2012;48(18):3342-3354.

5. Cortazar P, Geyer CE, Jr. Pathological complete response in neoadjuvant treatment of breast cancer. Ann Surg Oncol. 2015;22(5):1441-1446.

6. Hennessy BT, Hortobagyi GN, Rouzier R, et al. Outcome after pathologic complete eradication of cytologically proven breast cancer axillary node metastases following primary chemotherapy. J Clin Oncol. 2005;23(36):9304-9311.

7. Mougalian SS, Hernandez M, Lei X, et al. Ten-year outcomes of patients with breast cancer with cytologically confirmed axillary lymph node metastases and pathologic complete response after primary systemic chemotherapy. JAMA Oncol. 2016;2(4):508-516.

8. Cortazar P, Zhang L, Untch M, et al. Pathological complete response and long-term clinical benefit in breast cancer: the CTNeoBC pooled analysis. Lancet. 2014;384(9938):164-172.

9. Vila J, Mittendorf EA, Farante G, et al. Nomograms for predicting axillary response to neoadjuvant chemotherapy in clinically node-positive patients with breast cancer. Ann Surg Oncol. 2016; 23(11):3501-3509.

10. Bayraktar S, Gonzalez-Angulo AM, Lei X, et al. Efficacy of neoadjuvant therapy with trastuzumab concurrent with anthracycline- and nonanthracycline-based regimens for HER2-positive breast cancer. Cancer. 2012;118(9):2385-2393.

11. Dominici LS, Negron Gonzalez VM, Buzdar AU, et al. Cytologically proven axillary lymph node metastases are eradicated in patients receiving preoperative chemotherapy with concurrent trastuzumab for HER2-positive breast cancer. Cancer. 2010;116(12):2884-2889.

12. Rouzier R, Extra JM, Klijanienko J, et al. Incidence and prognostic significance of complete axillary downstaging after primary chemotherapy in breast cancer patients with T1 to T3 tumors and cytologically proven axillary metastatic lymph nodes. J Clin Oncol. 2002;20(5):1304-1310.

13. Kuerer HM, Sahin AA, Hunt KK, et al. Incidence and impact of documented eradication of breast cancer axillary lymph node metastases before surgery in patients treated with neoadjuvant chemotherapy. Ann Surg. 1999;230(1):72-78.

14. Tadros AB, Yang WT, Krishnamurthy $S$, et al. Identification of patients with documented pathologic complete response in the breast after neoadjuvant chemotherapy for omission of axillary surgery. JAMA Surg. 2017;152(7):665-670.

15. Straver ME, Rutgers EJ, Russell NS, et al. Towards rational axillary treatment in relation to neoadjuvant therapy in breast cancer. Eur J Cancer. 2009;45(13):2284-2292.

16. $\mathrm{CBO}$ Kwaliteitsinstituut voor de Gezondheidszorg. Vereniging van Integrale Kankercentra. Guideline 'Treatment of breast cancer' (Richtlijn 'Behandeling van het Mammacarcinoom'). 2008.

17. СВО Kwaliteitsinstituut voor de Gezondheidszorg. Vereniging van Integrale Kankercentra. Guideline 'Treatment of breast cancer' (Richtlijn 'Behandeling van het Mammacarcinoom'). 2012.

18. Giuliano AE, Connolly JL, Edge SB, et al. Breast cancer-major changes in the American Joint Committee on Cancer eighth edition cancer staging manual. CA Cancer J Clin. 2017;67(4):290-303.

19. van la Parra RF, Kuerer HM. Selective elimination of breast cancer surgery in exceptional responders: historical perspective and current trials. Breast Cancer Res. 2016;18(1):28. 
20. TrialRegister.nl. Towards omitting breast cancer surgery in patients without residual tumor after upfront chemotherapy. NTR6120. 2016. http://www.trialregister.nl/trialreg/admin/rctview.asp?TC=6120

21. ClinicalTrialregister.gov. Eliminating Breast Cancer Surgery in Exceptional Responders With Neoadjuvant Systemic Therapy. NCT02945579. 2017. https://clinicaltrials.gov/ct2/show/NCT02945579.

22. Kuerer HM, Rauch GM, Krishnamurthy $S$, et al. A clinical feasibility trial for identification of exceptional responders in whom breast cancer surgery can be eliminated following neoadjuvant systemic therapy. Ann Surg. 2018;267(5):946-951.

23. Hieken TJ, Boughey JC, Jones KN, Shah SS, Glazebrook KN. Imaging response and residual metastatic axillary lymph node disease after neoadjuvant chemotherapy for primary breast cancer. Ann Surg Oncol. 2013;20(10):3199-3204.

24. Weber JJ, Jochelson MS, Eaton A, et al. MRI and prediction of pathologic complete response in the breast and axilla after neoadjuvant chemotherapy for breast cancer. J Am Coll Surg. 2017;225(6):740746.

25. Caudle AS, Yang WT, Krishnamurthy S, et al. Improved axillary evaluation following neoadjuvant therapy for patients with node-positive breast cancer using selective evaluation of clipped nodes: Implementation of targeted axillary dissection. J Clin Oncol. 2016;34(10):1072-1078.

26. van Nijnatten TJA, Simons JM, Smidt ML, et al. A Novel less-invasive approach for axillary staging after neoadjuvant chemotherapy in patients with axillary node-positive breast cancer by combining radioactive iodine seed localization in the axilla with the sentinel node procedure (RISAS): A Dutch prospective multicenter validation study. Clin Breast Cancer. 2017;17(5):399-402. 



\section{CHAPTER}

Prognosis of residual axillary disease after neoadjuvant chemotherapy in clinically node-positive breast cancer patients: isolated tumor cells and micrometastases carry a better prognosis than macrometastases

JM Simons* TJA van Nijnatten*

M Moossdorff

$\mathrm{L}$ de Munck

MBI Lobbes

CC van der Pol

LB Koppert

EJT Luiten

ML Smidt

Breast Cancer Res Treat. 2017;163(1):159-166

*Authors contributed equally to this work 


\section{ABSTRACT}

\section{Purpose}

The aim of this study was to compare disease-free survival (DFS) and overall survival (OS) between clinically node-positive breast cancer patients, treated with neoadjuvant chemotherapy (NAC), with axillary pathologic complete response (ypNO), residual axillary isolated tumour cells or micrometastases (ypNitc/mi), and residual axillary macrometastases (ypN1-3).

\section{Methods}

All patients diagnosed with clinically node-positive primary invasive breast cancer treated with NAC and subsequent axillary lymph node dissection between 2005 and 2008 were retrospectively analyzed. Data were obtained from the Netherlands Cancer Registry. Patients were stratified by final pathological axillary status: ypNO, ypNitc/mi, or ypN1-3. The main outcome measures DFS and OS were analyzed using KaplanMeier survival analysis. Uni- and multivariable cox regression analyses were used to determine independent predictors for DFS and OS.

\section{Results}

A total of 1347 patients were included. Pathologic nodal status was ypNO in $22.2 \%$, ypNitc/mi in 3.8\%, and ypN1-3 in $74.0 \%$ of patients. Overall, 5-year DFS was $57.8 \%$ and mean OS was 7.4 years. DFS and OS were comparable between ypNO and $y p N i t c / m i \quad(H R \quad 1.38$ (0.40-4.79, $p=0.613)$ and $H R \quad 0.92 \quad(0.27-3.09, p=0.889)$, respectively), but significantly different between ypNO and ypN1-3 (HR 1.78 (1.06-3.00, $p=0.031$ ) and HR 1.70 (1.07-2.71, p=0.026), respectively).

\section{Conclusions}

Clinically node-positive patients, treated with NAC, with axillary nodal status ypNO or ypNitc/mi carry similar prognosis regarding DFS and OS. Axillary nodal status ypN1-3 is associated with a less favorable prognosis. Future studies should consider ypNO and $\mathrm{ypNitc/mi}$ as one entity. 


\section{INTRODUCTION}

Over the past 20 years, a trend toward a less invasive approach regarding the surgical management of the axilla in breast cancer patients has been observed. Nowadays, a sentinel lymph node biopsy (SLNB) has been widely adopted for staging of early-stage clinically node-negative breast cancer. ${ }^{1}$ In case of a sentinel lymph node (SLN) containing isolated tumour cells (ITCS) or micrometastases, a completion of axillary lymph node dissection (ALND) does not improve survival, nor does it reduce regional recurrence. Consequently, ALND following SLNB has been abandoned in these patients. $^{2-4}$ The ACOSOG Z0011 trial demonstrated no significant effect on prognosis when ALND is omitted in case of a SLN containing a limited number of metastases, even macrometastases, in patients treated with breast conserving therapy. ${ }^{3}$

In clinically node-positive ( $\mathrm{CN}+$ ) patients, ALND is regarded as standard surgical therapy. However, increased utilization of neoadjuvant chemotherapy (NAC) results in axillary pathologic complete response (pCR) in $30-40 \%$ of patients. ${ }^{5}$ Consequently, the value of ALND is topic of debate. Various studies demonstrated that axillary pCR after NAC is associated with improved prognosis. ${ }^{6-8}$ Residual axillary disease has a less favorable prognosis, but it is unknown whether different degrees of residual axillary disease (i.e., ITCs, micrometastases, macrometastases) all have similar prognosis.

Hence, the purpose of this study was to compare prognosis of axillary $p C R$, residual ITCs, or micrometastases and residual macrometastases in $\mathrm{cN}+$ patients treated with NAC.

\section{METHODS}

\section{Data collection}

In this study, all pathologically confirmed $\mathrm{cN}+$ patients diagnosed with primary invasive breast cancer and treated with NAC (with or without immunotherapy) followed by ALND between 2005 and 2008 were included. Exclusion criteria were synchronous breast cancer, primary surgical treatment, neoadjuvant radiation therapy, neoadjuvant endocrine therapy, unknown pathological nodal status, and distant metastases diagnosed within 91 days after primary breast cancer diagnosis. Patients who did not undergo ALND were also excluded.

Data were obtained from the Netherlands Cancer Registry (NCR), which is managed by the Netherlands Comprehensive Cancer Organisation (NCCO). The PALGA foundation (Pathologisch-Anatomisch Landelijk Geautomatiseerd Archief), a nationwide network and registry of histopathology and cytopathology diagnosis in the Netherlands, regularly submits reports of all diagnosed malignancies to the cancer registry. After 
notification, trained data collection registrars from the NCR extracted data from patients' records. Data were collected on age, tumour type, receptor status, surgical procedures, systemic therapy, adjuvant radiation therapy, and pathology results, including pathological TNM stage and tumour grade. During a 5-year period after initial diagnosis, the first of the following breast cancer events was registered: any local, regional, or contralateral recurrence or distant metastasis. Date of death or date of emigration was derived from the Municipal Personal Records Database (Basisregistratie Personen, BRP) and files until December 31, 2014 were analyzed. Patients were stratified into three subgroups according to final pathologic axillary nodal status after completion of NAC and definitive surgery: pCR (ypNO), residual isolated tumour cells or micrometastases (ypNitc/mi), and residual macrometastases (ypN1-3).

\section{Neoadjuvant chemotherapy (NAC) with/without immunotherapy}

During the study period, the Dutch national guideline of 2005 was in use. ${ }^{9}$ This guideline recommended chemotherapy regimens consisting of five courses 5 Fluorouracil, Epirubicin, Cyclophosphamide (FEC), or six courses of Taxotere, Adriamycin, and Cyclophosphamide (TAC). In case of Her2Neu receptor (Her2) amplification, targeted therapy (trastuzumab) was recommended in addition to chemotherapy.

\section{Statistics}

Statistical analyses were performed using Statistical Package for the Social Sciences software (Version 22, IBM, Armonk, New York, USA). General characteristics between the three subgroups were compared using Chi squared test for categorical data and One-way ANOVA for continuous data, after confirmation of Levene's test for equality of variances. If Levene's test demonstrated significant differences among the population variances, Kruskall-Wallis test was used.

DFS was defined as time from diagnosis to any local (including carcinoma in situ), regional, or contralateral recurrence, distant metastasis or mortality within 5 years after the primary diagnosis. Events occurring 0-91 days after diagnosis were considered synchronous to the original tumour and were not counted as recurrence. OS was defined as the time interval between date of diagnosis and date of death, date of first event, date of last follow-up, or date of emigration.

DFS and OS for the three subgroups were calculated with Kaplan-Meier curves and compared with the log-rank test. P-values (two-sided) $<0.05$ were considered statistically significant. Relevant clinicopathological variables associated with DFS and 
OS were examined using univariable and, where applicable, multivariable Cox proportional hazards regression, with Hazard Ratio (HR) and corresponding 95\% confidence intervals.

\section{RESULTS}

Between 2005 and 2008, 8176 patients were diagnosed with $\mathrm{cN}+$ breast cancer in the Netherlands. Patients were excluded for several reasons: 6553 patients underwent primary surgery; 204 patients did not undergo ALND; 9 patients were treated with neoadjuvant radiotherapy; 61 patients were treated with neoadjuvant endocrine therapy; and ypN status was unknown for 11 patients (Figure 8.1). A final total of 1347 patients were included for this study: $299 \mathrm{ypN0}, 51 \mathrm{ypNitc/mi}$ and $997 \mathrm{ypN1-3.}$

The incidence of $\mathrm{pCR}$ of the primary tumour was higher in patients with ypNO compared to ypNitc/mi and ypN1-3 patients (41.1\% vs. $19.6 \%$ and $7.1 \%$, respectively, $\mathrm{p}<0.001)$. Furthermore, lobular carcinoma was observed more often in patients with ypN1-3 than in ypNO and ypNitc/mi patients (9.4\% vs. $5.4 \%$ and $3.9 \%$, respectively, $\mathrm{p}=0.039$ ). Adjuvant radiation therapy was applied more often in ypN1-3 as compared to ypNO and ypNitc/mi patients $(92.1 \%$ vs. $80.9 \%$ and $80.4 \%$, respectively, $p<0.001$, Table 8.1).

Figure $8.1 \quad$ Flowchart of included patients.

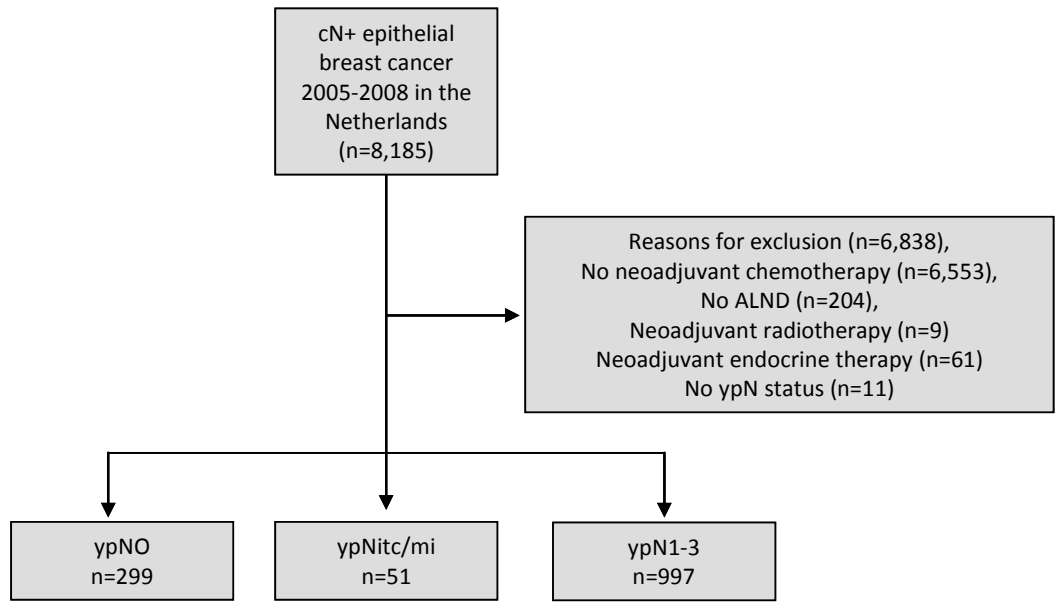

$\mathrm{cN}+$ clinically node positive status, SLNB sentinel lymph node biopsy, ALND axillary lymph node dissection, ypNO axillary pathologic complete response, ypNitc/mi axillary residual isolated tumour cells or micrometastases, ypN1-3 axillary residual macrometastases. 
Table 8.1 General characteristics.

\begin{tabular}{|c|c|c|c|c|}
\hline & $\begin{array}{c}\text { ypNO } \\
(n=299)\end{array}$ & $\begin{array}{c}\text { ypNitc/mi } \\
(n=51)\end{array}$ & $\begin{array}{l}\text { ypN1-3 } \\
\text { (n=997) }\end{array}$ & p-value \\
\hline Mean age (years) (range) & $\begin{array}{c}48.9 \\
(27-77) \\
\end{array}$ & $\begin{array}{c}48.2 \\
(29-81) \\
\end{array}$ & $\begin{array}{c}50.4 \\
(22-85) \\
\end{array}$ & 0.053 \\
\hline $\begin{array}{l}\text { Clinical T-stage (\%) } \\
\text { cT0-is } \\
\text { cT1-2 } \\
\text { cT3-4 } \\
\text { cTx }\end{array}$ & $\begin{array}{c}1(0.3) \\
138(47.0) \\
153(52.0) \\
7 \\
\end{array}$ & $\begin{array}{c}0 \\
26(52.0) \\
24(48.0) \\
1 \\
\end{array}$ & $\begin{array}{c}5(0.5) \\
458(47.0) \\
509(52.0) \\
25 \\
\end{array}$ & $\begin{array}{l}0.826 \\
0.780 \\
0.853\end{array}$ \\
\hline $\begin{array}{l}\text { Pathologic T-stage (\%) } \\
\text { ypT0-is } \\
\text { урT1-2 } \\
\text { ypT3-4 } \\
\text { Unknown } \\
\end{array}$ & $\begin{array}{c}123(50.8) \\
107(44.2) \\
12(5.0) \\
57 \\
\end{array}$ & $\begin{array}{c}10(26.3) \\
25(65.8) \\
3(7.9) \\
13 \\
\end{array}$ & $\begin{array}{c}71(8.5) \\
570(68.5) \\
191(23.0) \\
165 \\
\end{array}$ & $\begin{array}{l}<0.001 \\
<0.001 \\
<0.001\end{array}$ \\
\hline $\begin{array}{l}\text { Tumor grade }(\%)^{*} \\
1-2 \\
3 \\
\text { Unknown } \\
\end{array}$ & $\begin{array}{c}28(31.5) \\
61(68.5) \\
210 \\
\end{array}$ & $\begin{array}{c}7(50.0) \\
7(50.0) \\
37 \\
\end{array}$ & $\begin{array}{c}197(43.9) \\
252(56.1) \\
548 \\
\end{array}$ & $\begin{array}{c}<0.001 \\
0.051\end{array}$ \\
\hline $\begin{array}{l}\text { Tumor type (\%) } \\
\text { Ductal } \\
\text { Lobular } \\
\text { Other }\end{array}$ & $\begin{array}{l}227(75.9) \\
16(5.4) \\
56(18.7) \\
\end{array}$ & $\begin{array}{c}41(80.4) \\
2(3.9) \\
8(15.7) \\
\end{array}$ & $\begin{array}{c}746(74.8) \\
94(9.4) \\
157(15.8) \\
\end{array}$ & $\begin{array}{l}0.739 \\
0.039 \\
0.470 \\
\end{array}$ \\
\hline $\begin{array}{l}\text { Subtype (\%) } \\
\text { ER+PR+, Her2- } \\
\text { ER+PR-, Her2- } \\
\text { ER+Her2+ } \\
\text { ER-Her2+ } \\
\text { Triple negative } \\
\text { Unknown }\end{array}$ & $\begin{array}{c}35(12.8) \\
21(7.7) \\
47(17.1) \\
97(35.4) \\
74(27.0) \\
25 \\
\end{array}$ & $\begin{array}{c}11(22.5) \\
7(14.3) \\
20(40.8) \\
5(10.2) \\
6(12.2) \\
2 \\
\end{array}$ & $\begin{array}{c}349(37.3) \\
122(13.0) \\
152(16.3) \\
146(15.6) \\
166(17.8) \\
62 \\
\end{array}$ & $\begin{array}{c}<0.001 \\
0.035 \\
<0.001 \\
<0.001 \\
0.003\end{array}$ \\
\hline $\begin{array}{l}\text { Breast surgery (\%) } \\
\text { Breast conserving therapy } \\
\text { Mastectomy } \\
\text { Unknown }\end{array}$ & $\begin{array}{c}62(20.7) \\
237(79.3) \\
0 \\
\end{array}$ & $\begin{array}{c}12(23.5) \\
39(76.5) \\
0 \\
\end{array}$ & $\begin{array}{c}181(18.2) \\
816(81.8) \\
1 \\
\end{array}$ & $\begin{array}{l}0.421 \\
0.421\end{array}$ \\
\hline $\begin{array}{l}\text { Radiation therapy (\%) } \\
\text { Yes }\end{array}$ & $242(80.9)$ & $41(80.4)$ & $918(92.1)$ & $<0.001$ \\
\hline $\begin{array}{l}\text { Endocrine therapy to ER+ subtype (\%) } \\
\text { Yes }\end{array}$ & $95(84.8)$ & 35 (92.1) & $600(91.5)$ & 0.080 \\
\hline $\begin{array}{l}\text { Trastuzumab to Her2+ subtype (\%) } \\
\text { Yes }\end{array}$ & $127(92.0)$ & $19(76.0)$ & $247(89.2)$ & 0.057 \\
\hline
\end{tabular}

* In case of ypT0, tumor grade of biopsy prior to surgery was used. \# Including adenocarcinoma not otherwise specified, mucinous carcinoma, and mixed carcinoma. ypNO axillary pathologic complete response, ypNitc/mi axillary residual isolated tumor cells or micrometastases, ypN1-3 axillary residual macrometastases, cT-stage clinical tumor stage, pT-stage pathologic tumor stage, ER estrogen, PR progesterone, Her2 human epidermal growth factor receptor 2. 


\section{Disease-free survival}

Five-year follow-up was available for 944 patients $(70.1 \%$; $=206$ ypN0, n=34 ypNOi+/ypN1mi, $n=704$ ypN1-3): Recurrence occurred in 377 patients (39.9\%) and 22 patients died within 5 years (2.3\%). This resulted in a DFS event in $42.2 \%$ of the patients. DFS did not differ significantly between ypNO and ypNitc/mi (71.8 vs. $70.6 \%$, $p=0.978)$. When DFS was compared between ypNO and ypN1-3, a significant difference was found (71.8 vs. $53.4 \% ; p=0.049$ ) (Figure $8.2 \mathrm{~A}$ ).

Multivariable Cox regression analyses demonstrated no significant difference in DFS between ypNO and ypNitc/mi (HR 1.38 (0.40-4.79), p=0.613), but a significant difference in DFS between ypNO and ypN1-3 (HR 1.78 (1.06-3.00), p=0.031) (Table 8.2).

Furthermore, higher ypT stage (ypT 1-2: HR 2.73 (1.39-5.39), $P=0.004$ and ypT 3-4: HR 4.71 (2.35-9.43), $p<0.001)$ and higher tumour grade (HR $1.69(1.19-2.40), p=0.004)$ were identified as independent predictors of decreased DFS, whereas endocrine therapy was identified as independent predictor of increased DFS (HR 0.55 (0.36-0.85), $p=0.007)$.

Figure 8.2 Kaplan-Meier curves for disease-free (A) and overall survival (B), including number at risk.

A

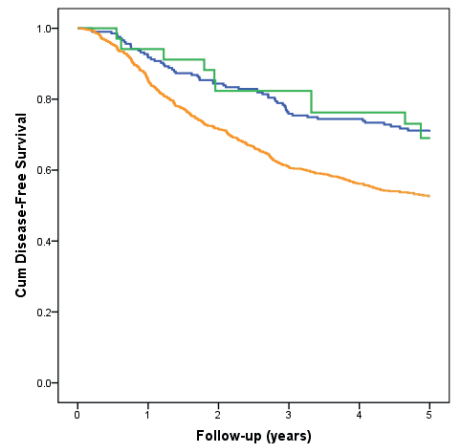

B

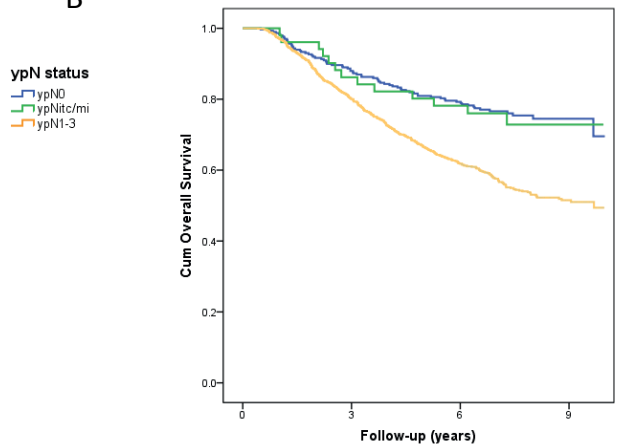

\begin{tabular}{|c|c|c|c|c|c|c|}
\hline$y p N O$ & 206 & 188 & 172 & 152 & 146 & 80 \\
\hline$y p N i t c / m i$ & 34 & 32 & 28 & 27 & 25 & 15 \\
\hline$y p N 1-3$ & 704 & 598 & 499 & 423 & 381 & 241 \\
\hline
\end{tabular}

\begin{tabular}{|c|c|c|c|c|}
\hline$y p N O$ & 299 & 263 & 236 & 43 \\
\hline$y p$ Nitc/mi & 51 & 43 & 38 & 9 \\
\hline$y p N 1-3$ & 997 & 795 & 612 & 109 \\
\hline
\end{tabular}

ypN status pathologic nodal status after neoadjuvant chemotherapy, ypNO axillary pathologic complete response, ypNitc/mi axillary residual isolated tumour cells or micrometastases, ypN1-3 axillary residual macrometastases. 
Table 8.2 Uni- and multivariable analyses of predictors of disease-free survival at 5 years.

\begin{tabular}{|c|c|c|c|c|}
\hline & \multicolumn{2}{|c|}{ Univariable analysis } & \multicolumn{2}{|c|}{ Multivariable analysis } \\
\hline & HR $(95 \% \mathrm{Cl})$ & p-value & HR $(95 \% \mathrm{Cl})$ & p-value \\
\hline $\begin{array}{l}\text { ypNO } \\
\text { ypNitc/mi } \\
\text { ypN1-3 }\end{array}$ & $\begin{array}{c}\text { Reference } \\
1.02(0.52-1.99) \\
1.89(1.43-2.50)\end{array}$ & $\begin{array}{c}0.964 \\
<0.001 \\
\end{array}$ & $\begin{array}{c}\text { Reference } \\
1.38(0.40-4.79) \\
1.78(1.06-3.00)\end{array}$ & $\begin{array}{l}0.613 \\
0.031\end{array}$ \\
\hline Age (per year increment) & $1.02(1.01-1.02)$ & 0.001 & $1.01(0.99-1.02)$ & 0.479 \\
\hline $\begin{array}{l}\text { ypT-stage } \\
\text { T0 or Tis } \\
\text { T1-2 } \\
\text { T3-4 }\end{array}$ & $\begin{array}{c}\text { Reference } \\
1.88(1.31-2.70) \\
3.74(2.53-5.54)\end{array}$ & $\begin{array}{c}0.001 \\
<0.001\end{array}$ & $\begin{array}{c}\text { Reference } \\
2.73(1.39-5.39) \\
4.71(2.35-9.43)\end{array}$ & $\begin{array}{c}0.004 \\
<0.001\end{array}$ \\
\hline $\begin{array}{l}\text { Tumor type } \\
\text { Ductal } \\
\text { Lobular } \\
\text { Other } \\
\end{array}$ & $\begin{array}{c}\text { Reference } \\
0.78(0.53-1.14) \\
0.89(0.69-1.15)\end{array}$ & $\begin{array}{l}0.193 \\
0.386 \\
\end{array}$ & $\begin{array}{c}\text { Reference } \\
1.11(0.59-2.07) \\
0.89(0.58-1.37)\end{array}$ & $\begin{array}{l}0.751 \\
0.595\end{array}$ \\
\hline $\begin{array}{c}\text { Tumor grade } \\
3 \text { vs. } 1-2 \\
\end{array}$ & $1.64(1.22-2.20)$ & 0.001 & $1.69(1.19-2.40)$ & 0.004 \\
\hline $\begin{array}{l}\text { Subtypes } \\
\text { ER+PR+Her2-: Yes vs. No } \\
\text { ER+PR-Her2-: Yes vs. No } \\
\text { ER+Her2+: Yes vs. No,* } \\
\text { ER-Her2+: Yes vs. No } \\
\text { Triple negative: Yes vs. No }\end{array}$ & $\begin{array}{l}0.63(0.50-0.80) \\
1.32(0.99-1.75) \\
0.63(0.47-0.85) \\
1.20(0.95-1.53) \\
1.94(1.46-2.32)\end{array}$ & $\begin{array}{c}<0.001 \\
0.057 \\
0.002 \\
0.129 \\
<0.001\end{array}$ & $1.16(0.69-1.93)$ & 0.577 \\
\hline $\begin{array}{c}\text { Trastuzumab } \\
\text { Yes vs. No } \\
\end{array}$ & $0.81(0.65-1.00)$ & 0.052 & $0.76(0.47-1.23)$ & 0.263 \\
\hline $\begin{array}{l}\text { Endocrine therapy } \\
\text { Yes vs. No }\end{array}$ & $0.57(0.47-0.69)$ & $<0.001$ & $0.55(0.36-0.85)$ & 0.007 \\
\hline $\begin{array}{l}\text { Radiation therapy } \\
\text { Yes vs. No }\end{array}$ & $1.09(0.78-1.52)$ & 0.626 & $0.74(0.45-1.23)$ & 0.251 \\
\hline
\end{tabular}

\# Excluded from multivariable analysis due to collinearity with endocrine therapy. ${ }^{*}$ Excluded from multivariable analysis due to collinearity with trastuzumab. ypNO axillary pathologic complete response, ypNitc/mi axillary residual isolated tumor cells or micrometastases, ypN1-3 axillary residual macrometastases, ypT-stage pathologic tumor stage after neo-adjuvant chemotherapy, ER estrogen, PR progesterone, Her2 human epidermal growth factor receptor 2, HR Hazard ratio.

\section{Overall survival}

Mean OS was 7.4 years (range 0.4-10 years): 8.3 years for ypN0, 8.2 years for $\mathrm{ypNitc/mi}$, and 7.0 years for ypN1-3 (Figure 8.2B). OS was comparable between ypN0 and $y p N i t c / m i ~(~ p=0.875)$. However, OS was significantly lower for ypN1-3 as compared to ypNO $(p=0.014)$.

Multivariable Cox regression analyses demonstrated no significant difference in OS between ypNO and ypNitc/mi (HR: 0.92 (0.27-3.09), p=0.889), but a significant difference in OS between ypNO and ypN1-3 (HR 1.70 (1.07-2.71), $p=0.026)$ (Table 8.3). Other independent predictors of decreased OS were higher ypT stage ((ypT 1-2: HR 2.40 (1.32-4.36), p=0.004) and (ypT 3-4: HR 4.38 (2.37-8.12), $p<0.001)$ ) and higher 
tumour grade (HR 1.72 (1.25-2.36), $\mathrm{p}=0.001)$. Furthermore, endocrine therapy (HR 0.49 (0.34-0.72), $\mathrm{p}<0.001)$ was identified as an independent predictor of increased OS.

Table 8.3 Uni- and multivariable analyses of predictors for overall survival.

\begin{tabular}{|c|c|c|c|c|}
\hline & \multicolumn{2}{|c|}{ Univariable analysis } & \multicolumn{2}{|c|}{ Multivariable analysis } \\
\hline & $\mathrm{HR}(95 \% \mathrm{Cl}$ & P-value & HR $(95 \% \mathrm{Cl})$ & p-value \\
\hline ypNO & Reference & & Reference & \\
\hline ypNitc/mi & $1.07(0.59-1.94)$ & 0.816 & $0.92(0.27-3.09)$ & 0.889 \\
\hline ypN1-3 & $2.07(1.62-2.67)$ & $<0.001$ & $1.70(1.07-2.71)$ & 0.026 \\
\hline Age (per year increment) & $1.02(1.02-1.03)$ & $<0.001$ & $1.01(1.00-1.02)$ & 0.082 \\
\hline \multicolumn{5}{|l|}{ ypT-stage } \\
\hline TO or Tis & Reference & & Reference & \\
\hline $\mathrm{T} 1-2$ & $1.60(1.19-2.15)$ & 0.002 & $2.40(1.32-4.36)$ & 0.004 \\
\hline T3-4 & $3.33(2.41-4.59)$ & $<0.001$ & $4.38(2.37-8.12)$ & $<0.001$ \\
\hline \multicolumn{5}{|l|}{ Tumor type } \\
\hline Ductal & Reference & & Reference & \\
\hline Lobular & $0.98(0.72-1.33)$ & 0.906 & $1.33(0.78-2.27)$ & 0.288 \\
\hline Other & $0.98(0.78-1.24)$ & 0.882 & $1.03(0.69-1.53)$ & 0.887 \\
\hline \multicolumn{5}{|l|}{ Tumor grade } \\
\hline 3 vs. $1-2$ & $1.84(1.41-2.40)$ & $<0.001$ & $1.72(1.25-2.36)$ & 0.001 \\
\hline \multicolumn{5}{|l|}{ Subtypes } \\
\hline ER+PR+Her2-: Yes vs No \# & $0.62(0.51-0.76)$ & $<0.001$ & & \\
\hline ER+PR-Her2-: Yes vs No $\#$ & $1.28(1.00-1.65)$ & 0.050 & & \\
\hline $\mathrm{ER}+\mathrm{Her} 2+:$ Yes vs $\mathrm{No}^{\#, *}$ & $0.58(0.43-0.73)$ & $<0.001$ & & \\
\hline ER-Her2+: Yes vs No ${ }^{*}$ & $1.15(0.93-1.42)$ & 0.201 & & \\
\hline Triple negative: Yes vs No & $2.10(1.73-2.56)$ & $<0.001$ & $1.38(0.90-2.13)$ & 0.145 \\
\hline \multicolumn{5}{|l|}{ Trastuzumab } \\
\hline Yes vs No & $0.72(0.59-0.88)$ & 0.001 & $0.70(0.46-1.07)$ & 0.101 \\
\hline \multicolumn{5}{|l|}{ Endocrine therapy } \\
\hline Yes vs No & $0.56(0.47-0.66)$ & $<0.001$ & $0.49(0.34-0.72)$ & $<0.001$ \\
\hline \multicolumn{5}{|l|}{ Radiation therapy } \\
\hline Yes vs No & $1.31(0.96-1.77)$ & 0.086 & $1.06(0.64-1.74)$ & 0.829 \\
\hline
\end{tabular}

\# Excluded from multivariable analysis due to collinearity with endocrine therapy. ${ }^{*}$ Excluded from multivariable analysis due to collinearity with trastuzumab. ypNO axillary pathologic complete response, ypNitc/mi axillary residual isolated tumor cells or micrometastases, ypN1-3 axillary residual macrometastases, ypT-stage pathologic tumor stage after neo-adjuvant chemotherapy, ER estrogen, PR progesterone, Her2 human epidermal growth factor receptor 2, HR Hazard ratio.

\section{DISCUSSION}

This is the first study comparing prognosis of ypNO with ypNitc/mi and ypN1-3 in cN+ breast cancer patients treated with NAC. It is well known that axillary $p C R$ is an important prognostic factor. ${ }^{6-8}$ Residual axillary disease after completion of NAC is associated with a less favourable prognosis. However, to our knowledge, this is the first study that compares the long-term effect of different degrees of residual disease 
on prognosis. Our study showed that ypNO and ypNitc/mi carry similar prognosis and that ypN1-3 carries a significantly different and less favourable prognosis in terms of DFS and OS.

Current guidelines still recommend to perform ALND in $\mathrm{CN}+$ patients following NAC irrespective of axillary response. ${ }^{10,11}$ However, $\mathrm{cN}+$ patients converting to axillary $\mathrm{pCR}$ after completion of NAC remain a topic of debate since they are not expected to benefit from ALND. A non-invasive technique to accurately diagnose $p C R$ is currently unavailable. Various minimally invasive procedures have been suggested for this purpose. The SLNB was studied extensively and its reliability seems questionable with a reported overall false negative rate (FNR) of $15.1 \%$ and negative predictive values (NPV) of $86 \%$ or lower. ${ }^{5}$ Other recently introduced minimally invasive techniques, the MARI procedure (Marking the Axillary lymph node with Radioactive lodine seeds) and TAD (Targeted Axillary Dissection), are promising with FNRs of 7 and $2 \%$, respectively. However, with only evidence available of single center studies comprising small cohorts that support these techniques it is not (yet) safe to implement them in clinical practice. $^{12,13}$

In our cohort, all patients underwent an ALND and thus our results do not directly support a change in surgical axillary treatment after the completion of NAC. Considering the comparable prognosis between ypNO and ypNitc/mi, our results do question whether ypNitc/mi may mimic ypNO more than residual axillary disease. Thus, when minimally invasive procedures prove to predict the status of the axilla accurately, the indications for omitting ALND may not just be limited to ypNO. Therefore, current research on reducing axillary management in $\mathrm{cN}+$ patients should not focus only on ypNO patients, but also on patients with ypNitc/mi. In future, ALND may be rendered as a procedure only to manage residual macrometastases.

In clinically node-negative patients in adjuvant setting, the SLNB with a relatively high FNR of about $8 \%$ is permitted since axillary recurrences are rare and previous studies have shown that not all axillary residual disease eventually converts to clinically overt disease. $^{2,3,14}$ This is in part effectuated by adjuvant therapy (i.e., radiation and/or systemic therapy) and by biological subtypes influencing recurrence patterns. In $\mathrm{cN}+$ patients, however, no studies have adequately evaluated prognostic impact of omitting ALND in case of residual axillary disease. Despite this, a trend toward replacing ALND by less invasive axillary staging procedures that are known to miss potentially therapy-resistant disease is already ongoing worldwide. Therefore, it is of utmost importance to prospectively collect data of these patients to detect potential influences on prognosis.

Since prognosis seems comparable between post-ALND ypNO and ypNitc/mi in cN+ patients treated with NAC, imaging might play an important role in axillary staging 
after NAC in the future. Since ITCS and micrometastases are not detectable on highresolution exams, such as MRI or 18F-FDG PET/CT, imaging techniques were considered inaccurate for nodal assessment after completion of NAC. Yet, with our current observations in mind, dedicated axillary imaging is re-entering the arena as a modality to non-invasively identify residual macrometastases rather than 'any' extent of residual disease (including ITCs and micrometastases).

The strength of the current study is the large cohort of patients that all underwent ALND after NAC. But our study also has several limitations. Subgroups ypNO and ypN13 comprised 299 and 997 patients, respectively, where subgroup ypNitc/mi comprised only 51 patients. Our ypNitc/mi subcohort was too small to explore the influence of single versus multiple tumour-positive lymph nodes on prognosis, and further studies are needed to explore this concept. Yet, this subset of patients can be considered unique since ypNitc/mi in $\mathrm{cN}+$ breast cancer is rare and a previously reported study included only a few ypNitc/mi patients. ${ }^{15}$

Furthermore, our cohort was treated up to a decade ago. In that time frame, different guidelines were effective, and therefore results should be interpreted carefully regarding current practice. For example, some Her2+ patients did not receive trastuzumab in our cohort (19.6\%), since trastuzumab was just introduced by that time.

Finally, our results are based on a retrospective study design. Consequently, details on additional radiation therapy could not be taken into account since radiation therapy fields were not recorded for each patient. Therefore, its influence on prognosis could not be explored in more detail.

In conclusion, our study showed that prognosis of $\mathrm{cN}+$ patients who receive NAC is affected by the degree of axillary residual disease as measured in ALND specimens. Prognosis of isolated tumour cells and micrometastases was comparable to prognosis of ypNO and more favorable than prognosis of macrometastases in terms of DFS and OS irrespective of tumour type. Ongoing and future studies should therefore consider ypNO and ypNitc/mi as one entity. Future research must explore which patients may safely receive a different, less invasive approach than the current standard of performing ALND after completion of NAC in all patients that were $\mathrm{CN}+$ prior to NAC. 


\section{REFERENCES}

1. Pilewskie ML, Morrow M. Management of the clinically node-negative axilla: what have we learned from the clinical trials? Oncology (Williston Park) 2014;28(5):371-8.

2. Galimberti V, Cole BF, Zurrida S, Viale G, Luini A, Veronesi P, Baratella P, Chifu C, Sargenti M, Intra M, Gentilini O, Mastropasqua MG, Mazzarol G, Massarut S, Garbay JR, Zgajnar J, Galatius H, Recalcati A, Littlejohn D, Bamert M, Colleoni M, Price KN, Regan MM, Goldhirsch A, Coates AS, Gelber RD, Veronesi U, International Breast Cancer Study Group Trial i. Axillary dissection versus no axillary dissection in patients with sentinel-node micrometastases (IBCSG 23-01): a phase 3 randomised controlled trial. Lancet Oncol 2013;14(4):297-305.

3. Giuliano AE, Ballman K, McCall L, Beitsch P, Whitworth PW, Blumencranz P, Leitch AM, Saha S, Morrow M, Hunt KK. Locoregional recurrence after sentinel lymph node dissection with or without axillary dissection in patients with sentinel lymph node metastases: long-term follow-up from the american college of surgeons oncology group (Alliance) ACOSOG Z0011 Randomized Trial. Ann Surg 2016;264(3):413-420.

4. Giuliano AE, Hunt KK, Ballman KV, Beitsch PD, Whitworth PW, Blumencranz PW, Leitch AM, Saha S, McCall LM, Morrow M. Axillary dissection vs no axillary dissection in women with invasive breast cancer and sentinel node metastasis: a randomized clinical trial. JAMA 2011;305(6):569-575.

5. van Nijnatten TJ, Schipper RJ, Lobbes MB, Nelemans PJ, Beets-Tan RG, Smidt ML. The diagnostic performance of sentinel lymph node biopsy in pathologically confirmed node positive breast cancer patients after neoadjuvant systemic therapy: a systematic review and meta-analysis. Eur J Surg Oncol 2015;41(10):1278-1287.

6. Mougalian SS, Hernandez M, Lei X, Lynch S, Kuerer HM, Symmans WF, Theriault RL, Fornage BD, Hsu L, Buchholz TA, Sahin AA, Hunt KK, Yang WT, Hortobagyi GN, Valero V. Ten-year outcomes of patients with breast cancer with cytologically confirmed axillary lymph node metastases and pathologic complete response after primary systemic chemotherapy. JAMA Oncol 2016;2(4):508-516.

7. Hennessy BT, Hortobagyi GN, Rouzier R, Kuerer H, Sneige N, Buzdar AU, Kau SW, Fornage B, Sahin A, Broglio K, Singletary SE, Valero V. Outcome after pathologic complete eradication of cytologically proven breast cancer axillary node metastases following primary chemotherapy. J Clin Oncol 2005;23(36):9304-9311.

8. Meric F, Mirza NQ, Buzdar AU, Hunt KK, Ames FC, Ross MI, Pollock RE, Newman LA, Feig BW, Strom EA, Buchholz TA, McNeese MD, Hortobagyi GN, Singletary SE. Prognostic implications of pathological lymph node status after preoperative chemotherapy for operable T3NOMO breast cancer. Ann Surg Oncol 2000;7(6):435-440.

9. $\mathrm{CBO}$ Kwaliteitsinstituut voor de Gezondheidszorg. Vereniging van Integrale Kankercentra. Guideline 'Treatment of breast cancer' (Richtlijn 'Behandeling van het Mammacarcinoom') 2005.

10. Gradishar WJ, Anderson BO, Balassanian R, Blair SL, Burstein HJ, Cyr A, Elias AD, Farrar WB, Forero A, Giordano SH, Goetz M, Goldstein LJ, Hudis CA, Isakoff SJ, Marcom PK, Mayer IA, McCormick B, Moran M, Patel SA, Pierce LJ, Reed EC, Salerno KE, Schwartzberg LS, Smith KL, Smith ML, Soliman H, Somlo G, Telli M, Ward JH, Shead DA, Kumar R. Invasive breast cancer version 1.2016, NCCN clinical practice guidelines in oncology. J Nat/ Compr Canc Netw 2016;14(3):324-354.

11. NABON (2012) National breast cancer guideline, Oncoline. https://www.oncoline.nl/breastcancer. Accessed 23 Dec 2016

12. Caudle AS, Yang WT, Krishnamurthy S, Mittendorf EA, Black DM, Gilcrease MZ, Bedrosian I, Hobbs BP, DeSnyder SM, Hwang RF, Adrada BE, Shaitelman SF, Chavez-MacGregor M, Smith BD, Candelaria RP, Babiera GV, Dogan BE, Santiago L, Hunt KK, Kuerer HM. Improved axillary evaluation following neoadjuvant therapy for patients with node-positive breast cancer using selective evaluation of clipped nodes: implementation of targeted axillary dissection. J Clin Oncol 2016;34(10):1072-1078.

13. Donker M, Straver ME, Wesseling J, Loo CE, Schot M, Drukker CA, van Tinteren H, Sonke GS, Rutgers EJ, Vrancken Peeters MJ. Marking axillary lymph nodes with radioactive iodine seeds for axillary staging after neoadjuvant systemic treatment in breast cancer patients: the MARI procedure. Ann Surg 2015;261(2):378-382. 
14. van der Ploeg IM, Nieweg OE, van Rijk MC, Valdes Olmos RA, Kroon BB. Axillary recurrence after a tumour-negative sentinel node biopsy in breast cancer patients: a systematic review and metaanalysis of the literature. Eur J Surg Oncol 2008;34(12):1277-84.

15. Boileau JF, Poirier B, Basik M, Holloway CM, Gaboury L, Sideris L, Meterissian S, Arnaout A, Brackstone M, McCready DR, Karp SE, Trop I, Lisbona A, Wright FC, Younan RJ, Provencher L, Patocskai E, Omeroglu A, Robidoux A. Sentinel node biopsy after neoadjuvant chemotherapy in biopsyproven node-positive breast cancer: the SN FNAC study. J Clin Oncol 2015;33(3):258-64. 



\section{CHAPTER}

Discussion and future perspectives 



\section{SUMMARY AND GENERAL DISCUSSION}

Neoadjuvant chemotherapy is commonly used in patients with locally advanced breast cancer and increasingly used in patients with early stage breast cancer. By downsizing disease, it allows for de-escalation of subsequent surgery of the breast and axilla. De-escalation of axillary surgery in breast cancer treatment is an ongoing trend. In search of the optimal less invasive axillary management after neoadjuvant chemotherapy, both improving quality of life by omitting unnecessary ALND and guaranteeing oncologic safety are of utmost importance.

This thesis demonstrated a lack of consensus regarding axillary management after neoadjuvant chemotherapy. The additional insights provided in this thesis aim to contribute to creating consensus and shaping response-based management of the axilla.

\section{Axillary staging in node negative breast cancer}

In cNO patients treated with adjuvant chemotherapy, SLND has been the technique for axillary staging since the 90s. As shown in Chapter 2, SLND has also increasingly replaced ALND over the past decade in $\mathrm{CNO}$ patients treated with neoadjuvant chemotherapy. Furthermore, a change in timing of SLND was detected, with SLND being increasingly performed after chemotherapy. Since neoadjuvant chemotherapy can eradicate axillary nodal disease, a positive SLND occurs less frequent after chemotherapy. ${ }^{1}$ This contributes to a further reduction in the need for ALND. This finding was also confirmed in Chapter 2: SLND performed after chemotherapy is less frequently followed by ALND. In a different population-based cohort study (Chapter 7), we assessed the correlation between breast PCR and axillary PCR. It was reported that $97.7 \%$ of cNO patients who achieved a pCR of the breast, also achieved a pCR of the axilla. ${ }^{2}$ This correlation was especially strong in Her2 positive and triple negative subtypes. These findings suggest that breast PCR may guide complete omission of axillary surgery in selected cNO patients treated with neoadjuvant chemotherapy.

In the adjuvant setting, indications for omitting ALND have extended from cNO/SLNnegative patients to $\mathrm{CNO} / \mathrm{SLN}$-positive patients ( $\leq 2$ positive SLNs), following 10 year follow-up results from trials like $20011 .^{3}$ Despite the fact that results of these trials only apply to patients treated with adjuvant systemic therapy, our study showed that completion ALND is also omitted in cNO/SLN-positive patients treated with neoadjuvant chemotherapy. In the ongoing SENOMAC trial, in which the need for completion ALND in SLN-positive patients is investigated, patients treated with neoadjuvant chemotherapy are also included. ${ }^{4}$ Breast-cancer specific survival and axillary recurrence rate will be compared between cNO/SLN positive patients treated with or without completion ALND. Results are expected by the end of 2029. In the 
currently recruiting POSNOC trial, patients are randomized between axillary treatment (ALND or axillary radiotherapy) and no axillary treatment. ${ }^{5}$ Patients treated with neoadjuvant chemotherapy are only eligible if the SLND is performed prior to chemotherapy. The primary endpoint is axillary recurrence at 5 years and results are expected in 2023.

\section{Axillary staging in node positive breast cancer}

In $\mathrm{cN}+$ patients treated with neoadjuvant chemotherapy, a less invasive procedure that accurately identifies axillary PCR is needed to enable safe of omission of ALND. Non-invasive techniques (i.e. imaging methods) are not able to accurately identify axillary pCR. Several less-invasive surgical techniques have been developed over the past years. In our systematic review and meta-analysis (Chapter 4$)^{6}$, we compared the diagnostic accuracy of three different staging procedures, i.e. SLND, excision of a pretreatment marked biopsy-proven positive lymph node (like the MARI-procedure) and a combination procedure (like Targeted Axillary Dissection). In all included studies, completion ALND was also performed. A total of 17 studies reported on SLND, compared to only one single center study for MARI and two for the combination procedure. SLND was associated with a rather poor overall accuracy (overall FNR of $17 \%$ and NPV $<86 \%$ ). Only when 3 or more SLNs were removed, SLND was statistically significantly associated with an improved FNR of $8 \%$. Unfortunately, only 1 or 2 SLNs can be identified in most of the patients. This renders SLND not feasible for clinical practice. One single-center study including 95 patients reported on the MARI procedure, which was associated with an FNR of 7\%. Current NPV's of both SLND and MARI (<86\% and $83 \%$, respectively) mean that in 1 out of every 6 patients with a negative test result, axillary residual disease is missed. ${ }^{6,7}$ The Targeted Axillary Dissection procedures, consisting of simultaneous SLND with a MARI/MARI-like procedure, were associated with a markedly improved NPV, ranging from $92 \%$ to $97 \%$. This means residual axillary disease is missed in only 1 in 12 to 33 patients. Although the combination procedure was associated with the highest accuracy, the lack of evidence (only 2 single-center studies consisting of 85 and 35 patients) to support this procedure has to be taken in mind. The ongoing multicenter RISAS trial ${ }^{8}$ in the Netherlands (Chapter 5), will prove whether the combination of SLND and MARI can safely replace ALND for axillary staging after neoadjuvant chemotherapy in cN+ patients. The total accrual of $225 \mathrm{cN}+$ patients is expected to be complete by the end of 2019.

Validation studies for the combination procedure (i.e. SLND combined with MARI or a MARI-like procedure always followed by ALND) are scarce, yet multiple studies have been published describing clinical practice experiences with a combination procedure. $^{6}$ In these studies, the outcome of the combination procedure guided 
decision-making on completion ALND and/or adjuvant regional radiotherapy. A wide variety of techniques is used for marking the biopsy proven positive lymph node: radiopaque clips $^{9-12}$, charcoal tattooing ${ }^{13}$, wires $^{11,14,15}$, iodine seeds ${ }^{8,16,17}$ and electromagnetic reflectors ${ }^{18}$. Apart from this, timing of marking differs: either primarily prior to chemotherapy (at the time of cytology/biopsy or during a second procedure once the pathology result is available) or secondary after chemotherapy (provided that a clip has been placed prior to chemotherapy to retrieve the right node). Since ALND was not always performed, these studies cannot report on the accuracy of the combination procedure, nevertheless they can provide useful insights. In Chapter 6 we reported on a retrospective cohort of $\mathrm{cN}+$ patients that underwent the combination procedure (the need for completion ALND was determined by the multidisciplinary tumour board). We found that the combination procedure was associated with significantly improved detection of residual disease over SLND only or MARI only. Further research has to define which combination procedure is most accurate, patient-friendly, and cost-effective.

In addition to the abovementioned less invasive procedures, a different less invasive axillary staging procedure involves preoperative fine needle aspiration of clipped nodes after neoadjuvant chemotherapy. Results of a prospective registry study including $50 \mathrm{cN}+$ patients were published in 2018: the identification rate was $92 \%$, the FNR $57.6 \%$ and the NPV $40.6 \%{ }^{19}$ Thus, contrary to the pre-chemotherapy setting in which FNA is highly accurate in identifying the presence of nodal metastasis, FNA is of no use in predicting the axillary treatment- response in $\mathrm{cN}+$ patients. Residual axillary disease is missed in more than half of the patients (of which implications on prognosis are yet unknown). Looking at the results from our survey in Chapter 3, FNA or biopsy of the axilla after chemotherapy in $\mathrm{cN}+$ patients was in fact reported to be part of clinical practice by some respondents. ${ }^{20}$ This is worrisome and again demonstrates the need for consensus and evidence based guidelines.

\section{Oncologic safety of omitting ALND}

In our retrospective analysis of $1347 \mathrm{cN}+$ patients treated with neoadjuvant chemotherapy (Chapter 8), we reported that isolated tumor cells and/or micrometastases after chemotherapy have a comparable prognosis to axillary pCR. ${ }^{21}$ This finding suggests that indications to omit ALND may be extended to patients with limited residual axillary disease. This could be relevant for the clinical use of the MARI procedure, since 2 of 5 false negative MARI results consisted of isolated tumor cells only. ${ }^{16}$ However, all included patients in our study underwent ALND with/without adjuvant regional radiotherapy and whether this contributed to our findings is unclear. Whether omission of ALND in patients with ypNO or only limited residual axillary disease is indeed safe in terms of long-term prognosis, has yet to be answered. 
Three studies reported on the prognosis of cNO/SLN-negative patients treated with neoadjuvant chemotherapy in whom completion ALND was not performed. In the retrospective cohort study by Nogi et al., axillary recurrence free survival was found to be similar between 147 patients treated with neoadjuvant chemotherapy and 849 patients treated with surgery first after a follow up period of 51 months ( $100 \%$ vs. $99.2 \%) .{ }^{22}$ Results of the prospective GANEA-2 trial showed a 3-year event free survival of $97.8 \%$ in $419 \mathrm{cNO}$ patients with a negative SLND and no completion ALND. ${ }^{23}$ In a retrospective cohort of $249 \mathrm{cNO}$ patients treated with neoadjuvant chemotherapy, SLN-negative patients (in whom ALND was omitted) had a significantly improved (distant) disease-free and overall survival compared to SLN-positive patients (in whom completion ALND was performed). ${ }^{24}$ To our knowledge, no studies have yet adequately assessed outcome of cNO/SLN-positive or $\mathrm{cN}+$ patients in whom ALND has been omitted after neoadjuvant chemotherapy. Nevertheless, in our populationbased cohort study of changes over time in axillary surgery, we found that ALND is already being omitted in cNO/SLN positive patients, and even in $\mathrm{cN}+$ patients with residual axillary disease. The decrease in ALND rates was not as large as in cNO and $\mathrm{cN}+$ patients with an ypNO status, but it was clearly present and gradually expanding. Positive lymph nodes detected after chemotherapy differ from positive lymph nodes detected at initial work-up. In the latter, patients still have to receive systemic treatment which contributes to locoregional control of the disease. Whereas patients treated with neoadjuvant chemotherapy already completed systemic therapy and positive lymph nodes indicate resistant disease. Therefore, leaving positive lymph nodes untreated after neoadjuvant chemotherapy, may in fact negatively impact locoregional control of disease. Studies assessing outcome in such patients in whom ALND is omitted are urgently needed. Additionally, studies comparing adjuvant regional radiotherapy with ALND are urgently needed. In our population-based study, simultaneously with a decrease in ALND, we noticed an increase in adjuvant regional radiotherapy in cN+ patients from 2011 to 2016. Currently, it is unknown whether adjuvant regional radiotherapy can safely replace ALND in such cases.

In patients in whom an ALND is still indicated, axillary reverse mapping (ARM) in combination with ALND may be considered to reduce morbidity ${ }^{25}$, since this procedure aims to preserve lymphatic drainage to the arm. In $\mathrm{cN}+$ patients treated with neoadjuvant chemotherapy, a significant decrease in metastases in the ARM lymph nodes has been reported. ${ }^{26}$ Furthermore, the chance of metastases in the ARM lymph nodes is very low in patients with a complete or partial nodal response on imaging after neoadjuvant chemotherapy. ${ }^{27}$ Future studies have to determine the long-term oncologic safety of ALND with ARM in breast cancer patients treated with neoadjuvant chemotherapy. 


\section{Main findings}

- In cNO patients, breast pCR is strongly correlated with ypNO and may guide complete omission of axillary surgery in selected patients. In $\mathrm{cN}+$ patients this correlation is also present, but weaker and therefore not relevant to clinical practice.

- The rate of ALND has declined significantly over the past decade in both cNO and $\mathrm{cN}+$ patients. This decline is also observed in patients with ypN+ disease, though implications on prognosis of leaving residual disease behind untreated are yet unknown. Whether adjuvant regional radiotherapy may safely replace ALND in such cases is yet unknown.

- The worldwide lack of consensus regarding axillary management after neoadjuvant chemotherapy in $\mathrm{cN}+$ patients results in a wide variety of practices: from a minimum 'wait and see' approach (in case of complete nodal response on imaging) to a 'most extensive' approach of ALND combined with adjuvant radiotherapy.

- When the accuracy of the three most commonly used less invasive axillary staging methods for $\mathrm{cN}+$ patients is compared, a combination procedure (like Targeted Axillary Dissection or RISAS) appears most accurate. Results of the RISAS trial have to be awaited to confirm this. When omission of ALND is already being considered in clinical practice, a combination procedure is the recommend staging procedure.

- In node positive breast cancer, patients with axillary pCR and limited residual axillary disease carry a comparable prognosis. Whether indications to omit ALND may be extended beyond axillary PCR remains thus far unknown, as data on longterm outcome are lacking.

\section{FUTURE PERSPECTIVES}

\section{Non-surgical approach}

The use of neoadjuvant chemotherapy has paved the way to explore a non-surgical approach for breast cancer. Previous attempts of omitting breast surgery after neoadjuvant chemotherapy resulted in unacceptably high rates of recurrent disease. ${ }^{28}$ With advances in imaging modalities and increased knowledge on subtypes and corresponding $\mathrm{PCR}$ rates, several studies are currently investigating the diagnostic accuracy of breast biopsy in identifying a pCR of the breast in "excellent responders". The ultimate goal is to forego unnecessary invasive surgery of the breast. In the currently accruing Dutch MICRA trial, breast-response is assessed with 8 ultrasoundguided biopsies in patients with a partial or complete breast response on MRI. ${ }^{29}$ 
Subsequently, either breast conserving surgery or mastectomy is performed to determine accuracy of the biopsies. A total of 525 patients is needed and all subtypes are included.

Another validation trial is ongoing in Germany; the RESPONDER trial. ${ }^{30}$ It is planned to enroll 600 patients (all subtypes included) with a partial or complete response on imaging that will undergo at least 12 image-guided vacuum assisted biopsies of the breast, subsequently followed by breast conserving surgery or mastectomy. The primary endpoint of the trial is the false negative rate of the biopsies.

While awaiting the results of these trials, MD Anderson Cancer Center has taken the next de-escalation step by evaluating the omission of surgery in excellent responders. In a prospective, multicenter trial, 50 patients with triple negative or HER2-positive breast cancer with a breast PCR as documented by 12 image-guided vacuum assisted core biopsies of the breast, will not undergo any subsequent surgery of the breast (Clinicaltrials.gov NCT02945579). All patients will undergo whole breast radiotherapy followed by external beam radiation therapy boost. The primary endpoint is the 6 months, 1, 2, 3, and 5-year biopsy confirmed ipsilateral breast tumor recurrence rate. If these trials determine safety of omission of breast surgery in selected patients, the next question is how to handle the axilla in such patients. Considering the strong correlation between breast $\mathrm{pCR}$ and $\mathrm{ypNO}^{2,31}$, a $\mathrm{pCR}$ of the breast may guide omission of axillary surgery in selected cNO patients. However, future trials have to determine if and in which patients that are eligible for omission of breast surgery, axillary surgery may also be omitted and in which patients axillary surgery should indeed be performed, even when surgery of the breast is omitted.

Currently, no trials are investigating a non-surgical approach of the axilla for patients treated with neoadjuvant chemotherapy specifically. This is apart from the previously mentioned study investigating FNA of the clipped node in the neoadjuvant setting, which reported a very low accuracy. ${ }^{19}$ In the adjuvant setting however, several trials are investigating omission of axillary surgery in cNO patients (since, following results from trials like Z0011, a positive SLN has no treatment consequences). In the Dutch prospective, multicenter BOOG 2013-08 trial $^{32}$, cT1-2cN0 patients that undergo breast conserving surgery are randomized between sentinel lymph node biopsy (+/- ALND) or no axillary surgery at all. So far, over $60 \%$ of the target sample size $(1092 / 1644)$ has been accrued. The primary end point is regional recurrence rate at five and ten years. The first results are expected in 2027. Patients treated with neoadjuvant chemotherapy are also eligible for this trial and stratified analysis will be performed. In the SOUND trial (Sentinel node vs. Observation after axillary Ultra-souND; Clinicaltrials.gov NCT02167490), a similar approach is investigated in a prospective, multicenter, randomized, controlled, non-inferiority trial. Patients with small breast 
tumours who are candidates for breast conserving surgery and have a negative preoperative axillary assessment (negative ultrasound or negative FNA) are randomized between no axillary surgery or SLND (+/- ALND). Overall, 1560 patients will be enrolled, and the primary endpoint is distant disease-free survival. In a third trial, the INSEMA trial (Clinicaltrials.gov NCT02466737), patients with cT1-2 breast cancer planned for breast conserving surgery are randomized to no SLND or SLND (+/ALND) in a 1 to 4 ratio. Approximately 6000 patients will be randomized, and the primary endpoint is invasive disease-free survival.

It is well known that the presence of axillary metastasis at diagnosis is an important prognostic factor, yet so is axillary treatment-response. Patients who achieve axillary PCR have improved survival over patients who do not achieve axillary PCR, irrespective of treatment-response in the breast. In patients with $\mathrm{pCR}$, we aim to avoid overtreatment. Simultaneously, we should provide appropriate adjuvant treatment in patients with residual disease. Future research should therefore also focus on this latter group, which has a dismal prognosis, to determine whether escalation rather than de-escalation of treatment may be necessary. Two recent trials, CREATE-X in HER2 negative patients ${ }^{33}$ and KATHERINE in HER2 positive patients ${ }^{34}$, reported an improved disease-free survival in patients with residual disease who were treated with adjuvant systemic therapy (capecitabine in CREATE-X and TDM- 1 in KATHERINE). These findings demonstrate the potential to improve long-term prognosis and the importance of accurate staging to guide adjuvant treatment.

\section{Imaging}

Currently, imaging has no place yet in identifying axillary $\mathrm{PCR}$. A systematic review on the diagnostic accuracy of different imaging techniques in $\mathrm{cN}+$ patients showed that both MRI and PET-CT are associated with high FNRs (up to 52\%) and low NPVs $(<86 \%){ }^{35}$ In the systematic review, axillary ultrasound turned out to be more accurate than MRI or PET-CT, but was still associated with high FNRs. Another disadvantage of imaging compared to surgical staging is the possibility of having a false positive finding, which results in overtreatment. Future studies should determine whether imaging and less invasive staging procedures may complement each other and when combined, may decrease the rate of both false negatives and false positives. New imaging techniques, such as the combination of hybrid PET-CT with MRI, may be more accurate and might be useful in assessing the axilla after neoadjuvant chemotherapy. A feasibility study reported that this technique may improve the diagnostic performance of the standard work-up for axillary staging in $\mathrm{cN}+$ patients. ${ }^{36}$ Future research has to determine if this may also apply for axillary staging in $\mathrm{cN}+$ patients after neoadjuvant chemotherapy. Our group is currently applying for funding for the 
OPTIMA trial. This trial aims to develop a deep learning approach for breast MRI in order to design a non-invasive prediction model for the extent of treatment-response.

\section{Prognostic implications of omitting ALND in node positive breast cancer}

As mentioned previously, data regarding long-term outcome of $\mathrm{cN}+$ patients in whom ALND is omitted after neoadjuvant chemotherapy are unavailable. Three currently accruing trials focus on this matter. In the Alliance 011202 trial (Clinicaltrials.gov NCT01901094), a total of 2918 cN1 patients with a clinically negative axilla on physical examination after chemotherapy and who have at least 1 positive SLN will be randomized between ALND with nodal radiotherapy (undissected axilla, supraclavicular and internal mammary nodes) or axillary and nodal radiotherapy (levels 1 to 3 of the axilla in addition to the undissected axilla, supraclavicular and internal mammary nodes). The primary and secondary outcomes are invasive breast cancer recurrence free survival, overall survival and Ipsilateral/local/regional invasive breast cancer recurrence at 5 years. First results of this trial are expected in 2024.

In the NSABP B51/RTOG 1304 trial (Clinicaltrials.gov NCT01872975), a total of 1636 cN1 patients with an axillary pCR after chemotherapy (documented by SLND or ALND) are randomized between regional nodal radiotherapy (undissected axilla, internal mammary nodes and ipsilateral supraclavicular fossa) or no regional nodal radiotherapy. The primary outcome is ipsilateral breast cancer recurrence free interval and amongst the secondary outcomes are overall survival and locoregional recurrence free interval. Patients will be followed for 10 years and results are expected in 2028. The outcomes of the NSABP B51/RTOG 1304 trial will inform us whether the poor accuracy of SLND in detecting $\mathrm{pCR}$ in $\mathrm{CN}+$ patients treated with neoadjuvant chemotherapy is nullified by extensive adjuvant axillary radiotherapy. In this way, undertreatment of potentially missed residual disease is prevented, yet it may result in overtreatment in case of a true pCR. With a highly accurate staging method, the risk of missing residual axillary disease is negligible, and adjuvant regional radiotherapy may no longer be of added value. In this light, results of the RISAS trial are much anticipated. The German TAXIS trial ${ }^{37}$ assesses long-term prognosis after performing a combination procedure. In this international trial, $1500 \mathrm{cN}+$ patients (treated with or without neoadjuvant chemotherapy) will undergo SLND with removal of the clipped node and palpably suspicious nodes. Patients with a positive node will then be randomized between radiotherapy levels 1-4 or ALND with radiotherapy of level 3-4 (Henke et al). The primary endpoint is disease-free survival at 20 years and these results are expected in 2043.

Future studies should not just focus on whether ALND can be safely replaced by regional radiotherapy, but also on identifying patients in whom both ALND and 
regional radiotherapy can be safely omitted. This will help further shape responsebased and patient-tailored management of the axilla.

A Dutch joined effort between our group and other dedicated breast cancer specialists has recently been granted funding to set up a multicenter, prospective, observational, registry study for $\mathrm{cN}+$ patients treated with neoadjuvant chemotherapy. In the Netherlands, $\mathrm{cN}+$ patients undergo either a less invasive staging procedure or an ALND depending on the institution where the patient is treated. Consequently, a nationwide cohort of $\mathrm{cN}+$ patients can be developed that allows for comparison between less and more invasive staging and treatment procedures. The primary aim is to determine 5- and 10-year disease-free, breast cancer specific and overall survival as well as axillary recurrence rates for the currently practiced less and more invasive staging and treatment procedures. In addition, the effect of the different procedures on quality of life will be assessed. The trial will commence in 2020. Since both retrospectively and prospectively collected data will be used to assess the endpoints, the first results of the trial are already expected in 2023. 


\section{REFERENCES}

1. Hunt KK, Yi M, Mittendorf EA, et al. Sentinel lymph node surgery after neoadjuvant chemotherapy is accurate and reduces the need for axillary dissection in breast cancer patients. Ann Surg. 2009;250(4):558-566.

2. Samiei S, van Nijnatten TJA, de Munck L, et al. Correlation between pathologic complete response in the breast and absence of axillary lymph node metastases after neoadjuvant systemic therapy. Ann Surg. 2018.

3. Giuliano AE, Ballman KV, McCall L, et al. Effect of axillary dissection vs no axillary dissection on 10year overall survival among women with invasive breast cancer and sentinel node metastasis: The ACOSOG Z0011 (Alliance) randomized clinical trial. JAMA. 2017;318(10):918-926.

4. de Boniface J, Frisell J, Andersson Y, et al. Survival and axillary recurrence following sentinel nodepositive breast cancer without completion axillary lymph node dissection: the randomized controlled SENOMAC trial. BMC Cancer. 2017;17(1):379.

5. Goyal A, Dodwell D. POSNOC: A randomised trial looking at axillary treatment in women with one or two sentinel nodes with macrometastases. Clin Oncol (R Coll Radiol). 2015;27(12):692-695.

6. Simons JM, van Nijnatten TJA, van der Pol CC, Luiten EJT, Koppert LB, Smidt ML. Diagnostic accuracy of different surgical procedures for axillary staging after neoadjuvant systemic therapy in nodepositive breast cancer: A systematic review and meta-analysis. Ann Surg. 2019;269(3):432-442.

7. Vugts G, Nieuwenhuijzen GA, Maaskant-Braat AJ, Schipper RJ, Smidt ML. Axillary response monitoring after neoadjuvant chemotherapy in breast cancer: Can we avoid the morbidity of axillary treatment? Ann Surg. 2016;263(2):e28-29.

8. van Nijnatten TJA, Simons JM, Smidt ML, et al. A Novel less-invasive approach for axillary staging after neoadjuvant chemotherapy in patients with axillary node-positive breast cancer by combining radioactive iodine seed localization in the axilla with the sentinel node procedure (RISAS): A Dutch prospective multicenter validation study. Clin Breast Cancer. 2017;17(5):399-402.

9. Nguyen TT, Hieken TJ, Glazebrook KN, Boughey JC. Localizing the clipped node in patients with nodepositive breast cancer treated with neoadjuvant chemotherapy: Early learning experience and challenges. Ann Surg Oncol. 2017;24(10):3011-3016.

10. Diego EJ, McAuliffe PF, Soran A, et al. Axillary staging after neoadjuvant chemotherapy for breast cancer: A pilot study combining sentinel lymph node biopsy with radioactive seed localization of pretreatment positive axillary lymph nodes. Ann Surg Oncol. 2016;23(5):1549-1553.

11. Dashevsky BZ, Altman A, Abe H, et al. Lymph node wire localization post-chemotherapy: Towards improving the false negative sentinel lymph node biopsy rate in breast cancer patients. Clin Imaging. 2018;48:69-73.

12. Siso C, de Torres J, Esgueva-Colmenarejo A, et al. Intraoperative ultrasound-guided excision of axillary clip in patients with node-positive breast cancer treated with neoadjuvant therapy (ILINA Trial) : A new tool to guide the excision of the clipped node after neoadjuvant treatment. Ann Surg Oncol. 2018;25(3):784-791.

13. Park S, Koo JS, Kim GM, et al. Feasibility of charcoal tattooing of cytology-proven metastatic axillary lymph node at diagnosis and sentinel lymph node biopsy after neoadjuvant chemotherapy in breast cancer patients. Cancer Res Treat. 2018;50(3):801-812.

14. Plecha D, Bai S, Patterson H, Thompson C, Shenk R. Improving the accuracy of axillary lymph node surgery in breast cancer with ultrasound-guided wire localization of biopsy proven metastatic lymph nodes. Ann Surg Oncol. 2015;22(13):4241-4246.

15. Hartmann S, Reimer T, Gerber B, Stubert J, Stengel B, Stachs A. Wire localization of clip-marked axillary lymph nodes in breast cancer patients treated with primary systemic therapy. Eur J Surg Oncol. 2018;44(9):1307-1311.

16. Donker M, Straver ME, Wesseling J, et al. Marking axillary lymph nodes with radioactive iodine seeds for axillary staging after neoadjuvant systemic treatment in breast cancer patients: the MARI procedure. Ann Surg. 2015;261(2):378-382. 
17. Caudle AS, Yang WT, Krishnamurthy S, et al. Improved axillary evaluation following neoadjuvant therapy for patients with node-positive breast cancer using selective evaluation of clipped nodes: implementation of targeted axillary dissection. J Clin Oncol. 2016;34(10):1072-1078.

18. Taback B, Jadeja P, Ha R. Enhanced axillary evaluation using reflector-guided sentinel lymph node biopsy: A prospective feasibility study and comparison with conventional lymphatic mapping techniques. Clin Breast Cancer. 2018;18(5):e869-e874.

19. Caudle AS, Kuerer HM, Krishnamurthy $S$, et al. Feasibility of fine-needle aspiration for assessing responses to chemotherapy in metastatic nodes marked with clips in breast cancer: A prospective registry study. Cancer. 2019;125(3):365-373.

20. Simons JM, Maaskant-Braat AJG, Luiten EJT, et al. Patterns of axillary staging and management in clinically node positive breast cancer patients treated with neoadjuvant systemic therapy: Results of a survey amongst breast cancer specialists. Eur J Surg Oncol. 2019.

21. van Nijnatten TJ, Simons JM, Moossdorff M, et al. Prognosis of residual axillary disease after neoadjuvant chemotherapy in clinically node-positive breast cancer patients: isolated tumor cells and micrometastases carry a better prognosis than macrometastases. Breast Cancer Res Treat. 2017;163(1):159-166.

22. Nogi H, Uchida K, Mimoto R, et al. Long-term follow-up of node-negative breast cancer patients evaluated via sentinel node biopsy after neoadjuvant chemotherapy. Clin Breast Cancer. 2017;17(8): 644-649.

23. Classe JM, Loaec C, Gimbergues $P$, et al. Sentinel lymph node biopsy without axillary lymphadenectomy after neoadjuvant chemotherapy is accurate and safe for selected patients: the GANEA 2 study. Breast Cancer Res Treat. 2019;173(2):343-352.

24. Galimberti V, Ribeiro Fontana SK, Maisonneuve P, et al. Sentinel node biopsy after neoadjuvant treatment in breast cancer: Five-year follow-up of patients with clinically node-negative or nodepositive disease before treatment. Eur J Surg Oncol. 2016;42(3):361-368.

25. Beek MA, Gobardhan PD, Klompenhouwer EG, et al. A patient- and assessor-blinded randomized controlled trial of axillary reverse mapping (ARM) in patients with early breast cancer. Eur J Surg Oncol. 2019.

26. Beek MA, Gobardhan PD, Klompenhouwer EG, Rutten HJ, Voogd AC, Luiten EJ. Axillary reverse mapping (ARM) in clinically node positive breast cancer patients. Eur J Surg Oncol. 2015;41(1):59-63.

27. Beek MA, Tetteroo E, Luiten EJ, et al. Clinical impact of breast MRI with regard to axillary reverse mapping in clinically node positive breast cancer patients following neo-adjuvant chemotherapy. Eur J Surg Oncol. 2016;42(5):672-678.

28. van la Parra RF, Kuerer HM. Selective elimination of breast cancer surgery in exceptional responders: historical perspective and current trials. Breast Cancer Res. 2016;18(1):28.

29. van der Noordaa MEM, van Duijnhoven FH, Loo CE, et al. Identifying pathologic complete response of the breast after neoadjuvant systemic therapy with ultrasound guided biopsy to eventually omit surgery: Study design and feasibility of the MICRA trial (Minimally Invasive Complete Response Assessment). Breast. 2018;40:76-81.

30. Heil J, Sinn P, Richter H, et al. RESPONDER - diagnosis of pathological complete response by vacuumassisted biopsy after neoadjuvant chemotherapy in breast cancer - a multicenter, confirmative, onearmed, intra-individually-controlled, open, diagnostic trial. BMC Cancer. 2018;18(1):851.

31. Tadros AB, Yang WT, Krishnamurthy $S$, et al. Identification of patients with documented pathologic complete response in the breast after neoadjuvant chemotherapy for omission of axillary surgery. JAMA Surg. 2017;152(7):665-670.

32. van Roozendaal LM, Vane MLG, van Dalen $\mathrm{T}$, et al. Clinically node negative breast cancer patients undergoing breast conserving therapy, sentinel lymph node procedure versus follow-up: a Dutch randomized controlled multicentre trial (BOOG 2013-08). BMC Cancer. 2017;17(1):459.

33. Masuda N, Lee SJ, Ohtani S, et al. Adjuvant capecitabine for breast cancer after preoperative chemotherapy. N Engl J Med. 2017;376(22):2147-2159.

34. von Minckwitz G, Huang CS, Mano MS, et al. Trastuzumab emtansine for residual invasive HER2positive breast cancer. N Engl J Med. 2019;380(7):617-628. 
35. Schipper RJ, Moossdorff M, Beets-Tan RGH, Smidt ML, Lobbes MBI. Noninvasive nodal restaging in clinically node positive breast cancer patients after neoadjuvant systemic therapy: a systematic review. Eur J Radiol. 2015;84(1):41-47.

36. van Nijnatten TJA, Goorts B, Voo S, et al. Added value of dedicated axillary hybrid 18F-FDG PET/MRI for improved axillary nodal staging in clinically node-positive breast cancer patients: a feasibility study. Eur J Nucl Med Mol Imaging. 2018;45(2):179-186.

37. Henke G, Knauer M, Ribi K, et al. Tailored axillary surgery with or without axillary lymph node dissection followed by radiotherapy in patients with clinically node-positive breast cancer (TAXIS): study protocol for a multicenter, randomized phase-III trial. Trials. 2018;19(1):667. 




\section{CHAPTER}

Nederlandse samenvatting 



\section{NEDERLANDSE SAMENVATTING}

Borstkanker is de meest voorkomende vorm van kanker onder vrouwen wereldwijd. De overlevingskansen van patiënten met borstkanker zijn over de afgelopen decennia aanzienlijk verbeterd ten gevolge van verbeteringen in screening, diagnose en behandeling. Daarnaast is de kwaliteit van leven van patiënten met borstkanker sterk verbeterd met de ontwikkeling van minder invasieve behandelopties. De kwaliteit van leven krijgt een steeds belangrijkere rol nu meer en meer patiënten borstkanker overleven.

De radicale mastectomie, waarin de gehele borst, borstspieren en alle okselklieren worden verwijderd, was van oudsher de standaardbehandeling voor borstkanker. Deze zeer invasieve ingreep werd na verloop van tijd aangepast tot de huidige standaard voor een mastectomie, waarin de borstspieren gespaard blijven. In de jaren '70 werd de borstsparende operatie geïntroduceerd. Deze operatie is een stuk minder invasief, en biedt wel dezelfde overlevingskansen als een mastectomie, mits de borstsparende operatie gevolgd wordt door aanvullende bestraling. De okselklierdissectie, waarbij alle okselklieren verwijderd worden, bleef de standaardbehandeling voor de oksel. De okselklierdissectie is een invasieve operatie die levenslange klachten van de arm kan geven, zoals lymfoedeem, functiestoornissen en pijnklachten. Deze klachten hebben een negatieve impact op de kwaliteit van leven van patiënten. Begin jaren '90 werd een minder invasieve okseloperatie geïntroduceerd: de schildwachtklierprocedure. Bij deze operatie worden alleen de okselklieren verwijderd die als eerste het lymfevocht uit de borst draineren in plaats van alle okselklieren. Wanneer deze 'poortwachtersklieren' vrij zijn van uitzaaiingen, is het heel waarschijnlijk dat de overige okselklieren ook vrij zijn van uitzaaiingen. Zodoende kunnen patiënten geselecteerd worden bij wie een okselklierdissectie achterwege gelaten kan worden. In sommige gevallen kan er zelfs veilig van een okselklierdissectie worden afgezien wanneer de schildwachtklieren slechts hele kleine (tot $2 \mathrm{~mm}$ ) uitzaaiingen bevatten. Wanneer in 2 of meer schildwachtklieren uitzaaiingen worden gevonden, is er in principe een indicatie voor een aanvullende okselklierdissectie. Patiënten met op het moment van diagnose al direct uitzaaiingen in de oksel (vastgesteld met behulp van echo en punctie), komen helaas niet in aanmerking voor de schildwachtklierprocedure. Bij hen bestaat er direct een indicatie voor een okselklierdissectie.

Naast de chirurgische behandeling, is chemotherapie een belangrijk onderdeel van de behandeling van borstkanker. Bij borstkanker werd chemotherapie traditioneel toegediend na een operatie. In de jaren '70 werd neoadjuvante chemotherapie geïntroduceerd, dat wil zeggen chemotherapie gegeven voorafgaand aan de operatie. Dit brengt een aantal voordelen met zich mee: men kan evalueren hoe de borstkanker reageert op de chemotherapie en het is mogelijk de tumor kleiner te maken. 
Tegenwoordig wordt neoadjuvante chemotherapie veelvuldig gebruikt. Niet alleen om niet-operabele borsttumoren operabel te maken, maar ook om reeds operabele borsttumoren kleiner te maken, zodat uiteindelijk minder borstweefsel verwijderd hoeft te worden. Neoadjuvante chemotherapie kan er zelfs voor zorgen dat álle kankercellen verdwijnen. Dit wordt een pathologisch complete respons genoemd. Patiënten met een pathologisch complete respons hebben waarschijnlijk geen baat bij een operatie. Helaas kunnen we op dit moment juist alleen maar middels een operatie met zekerheid vaststellen of iemand een pathologisch complete respons heeft bereikt. Om deze reden is men op zoek naar minder invasieve procedures om de invasieve operaties te kunnen vervangen in deze patiënten. In dit proefschrift ligt de focus op de operatie van de oksel. Het doel is om de meest optimale okseloperatie te identificeren. Deze operatie moet enerzijds zo minimaal invasief als mogelijk zijn om de kwaliteit van leven te verbeteren, anderzijds mag deze operatie geen kankercellen missen, want de oncologische veiligheid moet wel gegarandeerd blijven.

\section{Deel I De-escalatie van okselbehandeling na neoadjuvante chemotherapie}

In het eerste deel van dit proefschrift hebben we onderzoek gedaan naar de huidige trends in en gedachten over de okselbehandeling van borstkanker patiënten die behandeld zijn met neoadjuvante chemotherapie. In hoofdstuk 2 werd geëvalueerd hoe de okselbehandeling is veranderd over het afgelopen decennium. We konden aantonen dat het aantal okselklierdissecties sterk is afgenomen over de tijd. In kliernegatieve patiënten daalde het percentage okselklierdissecties van $89 \%$ tot $6 \%$ en in klierpositieve patiënten daalde dit van $99 \%$ tot $54 \%$. De dalende trend in het aantal okselklierdissecties werd daarnaast geobserveerd in zowel patiënten met een pathologisch complete respons als in patiënten bij wie nog wel positieve klieren gevonden werden na de chemotherapie. Toekomstige studies moeten uitwijzen of en hoe deze trend de lange termijn prognose beïnvloedt. In hoofdstuk 3 werden borstkanker specialisten gevraagd naar hun gedachten en gebruiken ten aanzien van de okselbehandeling in klierpositieve borstkanker die behandeld wordt met neoadjuvante chemotherapie. Er werd een vragenlijst onderzoek uitgevoerd waarop 310 voornamelijk Europese chirurgen hebben gereageerd. Een kleine meerderheid (57.3\%) gaf aan nog routinematig een okselklierdissectie te verrichten. Van de respondenten die niet routinematig een okselklierdissectie uitvoerden (42.7\%), maakte $85 \%$ gebruik van beeldvorming om het beleid ten aanzien van de okselbehandeling vast te stellen. In geval van een complete respons op beeldvorming, koos $95 \%$ voor een minder invasieve okseloperatie. In geval van een gedeeltelijke of geen respons op beeldvorming, koos respectievelijk $59 \%$ en $77 \%$ voor een okselklierdissectie. Middels deze vragenlijst studie hebben we kunnen aantonen dat 
er wereldwijd een gebrek aan consensus is en dat men behoefte heeft aan eenduidige, goed onderbouwde, richtlijnen.

\section{Deel II De zoektocht naar een accurate, minder invasieve, okseloperatie voor klierpositieve borstkanker}

Zoals reeds genoemd dient de meest optimale okseloperatie én zo minimaal invasief als mogelijk én zo accuraat als mogelijk te zijn. Zodoende kan de kwaliteit van leven verbeterd worden, zonder hierbij de oncologische veiligheid uit het oog te verliezen. In hoofdstuk 4 werd de diagnostische accuratesse van drie verschillende minder invasieve okseloperaties met elkaar vergeleken. De gouden standaard in alle meegenomen studies was de okselklierdissectie. Er werden in totaal 20 studies geïncludeerd: 17 studies over de schildwachtklierprocedure, 1 studie over de MARIprocedure en 2 studies over een okseloperatie waarin de schildwachtklierprocedure gecombineerd wordt met de MARI-procedure. In de MARI-procedure wordt de positieve klier voorafgaand aan de chemotherapie gemarkeerd met een klein, radioactief jodiumbronnetje. Dit bronnetje blijft tijdens de chemotherapie periode in de klier zitten. Na de chemotherapie wordt tijdens de operatie de klier met het jodiumbronnetje opgespoord en verwijderd. De schildwachtklierprocedure had een gepoolde identificatiewaarde van $89 \%$, een gepoolde fout-negatieve ratio van $17 \%$ en een negatief voorspellende waarde van $57 \%$ tot $86 \%$. De MARI-procedure had een identificatiewaarde van $97 \%$, een fout-negatieve ratio van $7 \%$ en een negatief voorspellende waarde van $83.3 \%$. De combinatie operatie had een identificatiewaarde van $100 \%$, een fout-negatieve ratio van $2 \%$ tot $4 \%$ en een negatief voorspellende waarde van $92 \%$ tot $97 \%$. De combinatie operatie was derhalve het meest accuraat, echter is de bewijskracht met slechts 2 studies beperkt en is er meer onderzoek nodig om dit te bevestigen. In hoofdstuk 5 werd het studieprotocol van de RISAS-studie beschreven. De RISAS-studie is een Nederlandse, prospectieve, multicenter, validatiestudie naar de diagnostische accuratesse van de okseloperatie waarin de schildwachtklierprocedure wordt gecombineerd met de MARI-procedure. Naar verwachting zullen eind 2019 in totaal 225 klierpositieve patiënten geïncludeerd zijn en volgen de resultaten medio 2020. Deze resultaten zullen bevestigen of een combinatie operatie daadwerkelijk het meest accuraat is. In hoofdstuk 6 werd het gebruik van de combinatie okseloperatie in de klinische praktijk onderzocht. Er werden retrospectief 139 klierpositieve patiënten uit vier ziekenhuizen geïncludeerd. Het combineren van de schildwachtklierprocedure en de MARI-procedure resulteerde in een hogere identificatie-waarde $(99.3 \%)$ dan wanneer alleen de schildwachtklierprocedure $(87.8 \%)$ of alleen de MARI-procedure zou zijn uitgevoerd (92.8\%). Met de combinatie operatie was ook de detectie van nog resterende positieve klieren beter. 


\section{Deel III Voorspellen van restziekte in de oksel en implicaties voor de prognose}

In hoofdstuk 7 werd de correlatie tussen een pathologisch complete respons van de borst en de oksel onderzocht in een cohort van 4084 patiënten. In kliernegatieve borstkanker was deze correlatie sterk: van de patiënten met een pathologisch complete response van de borst had $\mathbf{9 7 . 7 \%}$ een tumorvrije oksel. In klierpositieve borstkanker was deze correlatie veel minder sterk: van de patiënten met een pathologisch complete respons van de borst had slechts $45 \%$ dit ook in de oksel. Toekomstig onderzoek zal moeten uitwijzen of de respons in de borst mogelijkheden biedt om van een okseloperatie af te zien in kliernegatieve patiënten. In hoofdstuk 8 werd de prognose van klierpositieve patiënten die behandeld zijn met neoadjuvante chemotherapie beoordeeld op basis van de klierstatus na chemotherapie. Drie groepen werden met elkaar vergeleken: patiënten met een complete respons, patiënten met slechts geïsoleerde tumorcellen of micrometastasen en patiënten met macrometastasen. In een cohort van 1347 patiënten werd aangetoond dat patiënten met geïsoleerde tumorcellen of micrometastasen een vergelijkbare prognose hadden als patiënten met een complete respons (ziektevrije-overleving: hazard ratio (HR) 1.38 (0.40-4.79), $p=0.613$; totale overleving: HR 0.92 (0.27-3.09), $p=0.889)$. Patiënten met macrometastasen hadden een significant minder gunstige prognose dan patiënten met een complete respons (ziektevrije-overleving: HR 1.78 (1.06-3.00), $p=0.031$; totale overleving: HR 1.70 (1.07-2.71), p=0.026). In de toekomst kunnen een pathologisch complete respons en geïsoleerde tumorcellen of micrometastasen mogelijk als één entiteit beschouwd worden. 


APPENDICES

Valorisation

Ediorial (NTvO 2017;14:53-4)

List of publications

Acknowledgements | Dankwoord

Curriculum Vitae 



\section{VALORISATION}

Breast cancer is the most commonly diagnosed cancer amongst women worldwide. In the Netherlands, approximately 16.000 patients are diagnosed with invasive breast cancer each year. Survival rates of these patients have increased substantially over the past decades and are still improving with the development of new therapies (such as immunotherapy). With increasing numbers of breast cancer survivors, improving quality of life becomes more and more important. About $20 \%$ of newly diagnosed patients are treated with neoadjuvant chemotherapy and these rates increased gradually over the past years. Currently, there is much debate on what constitutes the optimal axillary management in patients treated with neoadjuvant chemotherapy. On the one hand, specialists are striving for a least invasive strategy, on the other hand, specialists can't justify improving quality of life at the expense of oncologic safety. The aim of this thesis was to help shape response-based axillary management.

\section{Relevance of scientific results in this thesis}

This thesis evaluated current practices and trends regarding axillary management in patients with breast cancer treated with neoadjuvant chemotherapy. Especially in node positive breast cancer, the management of the axilla is an area of controversy. Traditionally, an axillary lymph node dissection was always performed. However, when clinically node positive patients are treated with neoadjuvant chemotherapy, a substantial part achieves a pathologic complete response of the axilla. These patients will probably not benefit from an axillary lymph node dissection. This thesis therefore focused on identifying the most optimal less invasive surgical procedure for axillary staging after neoadjuvant chemotherapy in node positive breast cancer patients. The systematic review and meta-analysis we performed, showed that a combination of sentinel lymph node biopsy with the MARI-procedure is the most optimal procedure in terms of identification rate and accuracy (false negative rate and negative predictive value). Importantly, only 2 studies addressed the accuracy of a combination procedure and only 1 study that of the MARI procedure, compared to 17 studies for the sentinel lymph node biopsy. Despite convincing evidence, axillary lymph node dissection is already being replaced by different less invasive procedures in daily practice at some institutions. This also occurs in the Netherlands, as we confirmed in chapter 2 of this thesis. To take optimal use of this ongoing trend, we evaluated a cohort of patients in whom axillary lymph node dissection is already being replaced by a combination procedure in daily practice. In this study, we found that the sentinel lymph node biopsy and MARI procedure complement each other, resulting in an improved identification rate and improved detection of residual axillary disease. Both the results of the systematic review and this cohort study, provide valuable considerations for specialists who already have implemented or who are about to 
start implementing a less invasive staging procedure in daily practice. Based on the findings of these two studies, performing a combination procedure is recommended instead of performing either sentinel lymph node biopsy or the MARI procedure alone when omission of axillary lymph node dissection is considered.

\section{Target population}

The results of this thesis provide breast cancer specialists with insights in current practices worldwide regarding axillary management in patients treated with neoadjuvant chemotherapy. It furthermore provides steppingstones for specialists to help shape their local protocols regarding management of the axilla. Apart from this, the results of this thesis are helpful for patients diagnosed with breast cancer. The results can be used to inform patients accordingly and may aid in shared-decision making. While patients should be informed on the side effects of axillary lymph node dissection, they certainly should also be informed on the lack of evidence regarding oncologic safety of less invasive staging procedures. With the results of this thesis, we furthermore demonstrated that patients may receive a different axillary management plan dependent on where or by whom they are treated. Patients should be aware of this and informed accordingly by the treating specialist. These results stress the importance of continuing to join efforts to encourage patients to participate in clinical trials or registry studies. Ultimately, evidence-based guidelines can be determined to provided patients with a personalized - yet consistent amongst institutions - axillary staging and management plan that is optimal in terms of quality of life and oncologic safety.

\section{Innovation and future}

Although combining sentinel lymph node biopsy with a MARI-like procedure has been reported before, the RISAS trial is the first trial to validate such a combination procedure following a prospective and multicenter study design. Furthermore, a large cohort of 225 patients will be included. The results of this trial will confirm whether a combination procedure is indeed the most accurate less invasive axillary staging procedure for node positive breast cancer patients treated with neoadjuvant chemotherapy. If this procedure is proven to be accurate, these results will be implemented in guidelines worldwide. Consequently, more patients can benefit from a less invasive procedure and be spared the high risk of comorbidity that is associated with axillary lymph node dissection. Future research has to determine what kind of combination procedure is most optimal in terms of accuracy, cost-effectiveness and patient-friendliness. The MINIMAX trial, which will start accruing in the near future, will take the next step in providing evidence on the appropriate staging and treatment procedures concerning prognosis and quality of life. A less invasive staging procedure 
is already standard of care for $\mathrm{cN}+$ patients treated with NST in many institutions. In other institutions the axillary lymph node dissection is still routinely performed. Consequently, a nationwide cohort of $\mathrm{cN}+$ patients can be developed to compare less and more invasive axillary staging and treatment procedures. Therefore, a multicenter, retro- and prospective, observational, registry study will be initiated in $\mathrm{cN}+$ breast cancer patients treated with NST in the Netherlands. The primary aim is to determine five- and ten-year disease-free survival, breast cancer-specific survival, overall survival, and axillary recurrence rates, for the less and more invasive axillary staging and treatment procedures. The secondary aim is to determine their effect on QoL, assessed at baseline, at one year and at five years after diagnosis. The first results of the MINIMAX trial are expected in 2023. 



\section{OKSELBEHANDELING VAN PRIMAIR KLIERPOSITIEF MAMMACARCINOOM NA NEOADJUVANTE SYSTEMISCHE THERAPIE: IS MINDER WEL VEILIG?}

Editorial bij de bijdrage van M.E.M. van der Noordaa, F.H. van Duijnhoven en M.T.F.D. Vrancken Peeters, getiteld 'Minder okselklierdissecties bij borstkankerpatiënten na neoadjuvante systemische therapie door gebruik van PET/CT en de MARI-procedure'

JM Simons, LB Koppert, TJA van Nijnatten, ML Smidt, CC van der Pol, EJT Luiten (NTvO 2017;14:53-4)

\section{Inleiding}

De-escalatie van okselbehandeling bij patiënten met axillaire lymfekliermetastasen is zeer actueel. Het groeiende aantal patiënten met axillaire pathologisch complete respons ( $p C R$ ) dankzij neoadjuvante systemische therapie (NST) vereist een minder invasieve stadiëringsmethode dan de conventionele okselklierdissectie (OKD) met bijkomende morbiditeit. Van der Noordaa et al. presenteren een protocol waarbij de uitslag van PET/CT voorafgaand aan NST gecombineerd met de MARI-procedure na NST bepalen of en zo ja, welke vorm van aanvullende axillaire therapie een patiënt ontvangt. Dit zou resulteren in $80 \%$ correcte behandeling, $16 \%$ overbehandeling en $3 \%$ onderbehandeling. Het NKI is een van de toonaangevende instituten op het gebied van mammachirurgie en het ontwikkelen van minder invasieve behandelmethoden. De vraag is echter of het voorgestelde protocol niet te voorbarig is? En of dit protocol niet alleen morbiditeit reduceert, maar ook de oncologische veiligheid?

\section{De MARI-procedure}

In de unicentrische studie waarin MARI is gevalideerd werden 100 patiënten geïncludeerd, waarvan er bij $26 \%$ pCR werd bereikt. ${ }^{1}$ Het percentage fout-negatieven bedroeg $7 \%$. Echter, met name de negatief-voorspellende waarde (NVW) is belangrijk bij validatie van diagnostische procedures en betreft hier 83\%: bij 1 op de 6 patiënten met een negatieve MARI-klier is toch restziekte aanwezig. ${ }^{2}$ Daarnaast is het onbekend of de MARI-procedure, vanwege de leercurve, vergelijkbare resultaten zal behalen in andere centra. De combinatie van een soortgelijke procedure als MARI met de schildwachtklierprocedure, de 'targeted axillary dissection', wordt kort benoemd als alternatieve stadiëringsmethode. ${ }^{3}$ In een unicentrische, retrospectieve studie met 85 patiënten werd een NVW van $97 \%$ gerapporteerd: de kans op restziekte is daarmee nog slechts 1 op 33 bij een negatieve testuitslag. Bij 23\% kwam de gemarkeerde klier niet overeen met (één van) de schildwachtklier(en). Gezien het beperkte bewijs voor deze veelbelovende methode is in Nederland de multicentrische RISAS-studie gestart 
(KWF-grant NCT02800317) om bij 225 patiënten de MARI-procedure gecombineerd met de schildwachtklierprocedure te valideren. In deze studie ondergaan alle patiënten aansluitend een completerende okselklierdissectie.

\section{De behandelmogelijkheden in het gepresenteerde protocol}

Alleen patiënten met >3 FDG-avide klieren op PET/CT en een positieve MARI-klier ondergaan een OKD. Naast de OKD worden radiotherapie en geen behandeling als aanvullend alternatief genoemd, maar is deze 'sparende okselbehandeling' onderbouwd? Is het terecht om niet alleen bij pCR maar ook bij beperkte restziekte voor een sparende okselbehandeling te kiezen? Kan radiotherapie veilig de aanvullende OKD vervangen bij deze geselecteerde patiënten? En krijgen in dit protocol wellicht juist de lager risico hormoongevoelige patiënten, van wie bekend is dat zij het minst vaak pCR vertonen, de meest uitgebreide okselbehandeling? Al deze vragen blijven tot dusver onbeantwoord. Het gepresenteerde protocol is inspirerend, maar vooralsnog onvoldoende wetenschappelijk onderbouwd. $\mathrm{Er}$ wordt vooruitgelopen op resultaten van lopende studies (NSABP B-51/RTOG 1304, Alliance A11002002). Daarnaast worden resultaten van 'adjuvante setting'-studies (AMAROS) geëxtrapoleerd naar de patiëntenpopulatie met primair axillaire lymfekliermetastasen in 'neoadjuvante setting'. De stelling dat $80 \%$ van de patiënten volgens het nieuwe protocol correct zou zijn behandeld is wellicht te voorbarig, aangezien de langetermijnresultaten vooralsnog onbekend zijn. Centra die dit protocol toepassen moeten sterk overwegen patiënten in een prospectieve database te includeren om deze resultaten inzichtelijk te maken.

\section{Conclusie}

Concluderend is er behoefte aan okselbehandeling gestuurd op NST-respons. Het protocol geopperd door Van der Noordaa et al. maakt hierin een grote stap voorwaarts in het streven naar de-escalatie. Wij benadrukken het belang van verkrijgen van meer wetenschappelijk bewijs en het prospectief registreren van patiënten bij wie een OKD en/of radiotherapie reeds achterwege wordt gelaten op basis van NST-respons. Daarnaast kan deelname aan de RISAS-studie worden overwogen. Zodoende kan straks betrouwbaar worden vastgesteld welke stadiëringsprocedure voldoende accuraat is en wat de aanvullende okselbehandeling moet zijn in geval van restziekte. Vooralsnog blijft dit echter onbeantwoord. 


\section{REFERENCES}

1. Donker M, Straver ME, Wesseling J, et al. Marking axillary lymph nodes with radioactive iodine seeds for axillary staging after neoadjuvant systemic treatment in breast cancer patients: the MARI procedure. Ann Surg. 2015;261(2):378-382.

2. Vugts G, Nieuwenhuijzen GA, Maaskant-Braat AJ, Schipper RJ, Smidt ML. Axillary Response Monitoring After Neoadjuvant Chemotherapy in Breast Cancer: Can We Avoid the Morbidity of Axillary Treatment? Ann Surg. 2016;263(2):e28-29.

3. Caudle AS, Yang WT, Krishnamurthy S, et al. Improved Axillary Evaluation Following Neoadjuvant Therapy for Patients With Node-Positive Breast Cancer Using Selective Evaluation of Clipped Nodes: Implementation of Targeted Axillary Dissection. J Clin Oncol. 2016;34(10):1072-1078. 
Appendices 


\section{LIST OF PUBLICATIONS}

\section{This thesis}

1. JM Simons, MLMA v Pelt, AWKS Marinelli, ME Straver, AM Zeillemaker, LM Pereira Arias-Bouda, TJA v Nijnatten, LB Koppert, ML Smidt, EJT Luiten, CC vd Pol "Detection of residual axillary disease after neoadjuvant systemic therapy based on excision of both the pretreatment marked positive lymph node and sentinel lymph nodes"

Br J Surg. 2019 Nov. Doi: 10.1002/bjs.11320.

2. JM Simons, A Maaskant, EJT Luiten, M Leidenius, TJA v Nijnatten, P Boelens, LB Koppert, CC vd Pol, CJH vd Velde, R Audisio, ML Smidt

"Practice patterns for axillary staging and management of clinically node positive breast cancer patients: results of a survey amongst breast cancer specialists"

EJSO. 2019 Aug. Doi: 19.1916/j.ejso.2019.08.012.

3. S Samiei, TJA v Nijnatten, L d Munck, KBMI Keymeulen, JM Simons, LFS Kooreman, S Siesling, MBI Lobbes MD, ML Smidt

"Correlation between Pathologic Complete Response in the Breast and absence of Axillary Lymph Node Metastases after Neoadjuvant Systemic Therapy"

Ann Surg. 2018 Dec. Doi: 10.1097/SLA.0000000000003126.

4. JM Simons, TJA v Nijnatten, CC vd Pol, EJT Luiten, LB Koppert, ML Smidt

"Diagnostic accuracy of different surgical procedures for axillary staging after neoadjuvant systemic therapy in node-positive breast cancer: a systemic review and meta-analysis"

Ann Surg. 2018 Oct. Doi: 10.1097/SLA.0000000000003075.

5. JM Simons*, TJA v Nijnatten*, M Moossdorff, L d Munck, MBI Lobbes, CC vd Pol, LB Koppert, EJT Luiten, ML Smidt

"Prognosis of residual axillary disease after neoadjuvant systemic therapy in clinically node positive breast cancer patients: isolated tumor cells and micrometastases carry a better prognosis than macrometastases"

Breast Cancer Res Treat. 2017 Feb 17. Doi: 10.1007/s10549-017-4157-0.

*Co-first authorship, reflected in alphabetical order on publication 
6. JM Simons*, TJA $\vee$ Nijnatten*, ML Smidt, CC vd Pol, PJ $\vee$ Diest, A Jager, D v Klaveren, BLR Kam, MBI Lobbes, M d Boer, C Verhoef, LB Koppert, EJT Luiten "A novel less invasive approach for axillary staging after neoadjuvant chemotherapy in patients with axillary node positive breast cancer by combining Radioactive lodine Seed localisation in the Axilla with the Sentinel node procedure (RISAS): a Dutch prospective multicentre validation study"

Clin Breast Cancer. 2017 Apr 19. Doi: 10.1016/j.clbc.2017.04.006.

*Co-first authorship, reflected in alphabetical order on publication

7. JM Simons, LB Koppert, TJA v Nijnatten, ML Smidt, CC vd Pol, EJT Luiten "Okselbehandeling van primair klierpositief mammacarcinoom na neoadjuvante systemische therapie: is minder wel veilig?"

Ned Tijdschr Oncol 2017;14:53-4.

8. JM Simons, A Maaskant, EJT Luiten, M Leidenius, TJA v Nijnatten, P Boelens, LB Koppert, CC vd Pol, CJH vd Velde, R Audisio, ML Smidt

"Reply to: Sentinel node biopsy after neoadjuvant chemotherapy for breast cancer in patients with pre-treatment node-positive: recommendation to optimize the performance"

Accepted in EJSO.

9. JM Simons, S Siesling, H d Wilt, TJA v Nijnatten, CC vd Pol, LB Koppert, EJT Luiten, ML Smidt

"Trends for omission of axillary lymph node dissection in breast cancer patients treated in the neoadjuvant era: a Dutch population-based study"

Submitted.

\section{Other}

10. MEH Jaspers, CM Stekelenburg, JM Simons, KM Brouwer, E vd Kerckhove, E Middelkoop, PPM v Zuijlen

"Vascularization in hypertrophic scars evaluated by different tools: an explorative study"

Burns. 2017 Jan 30. pii: S0305-4179(17)30034-7. doi: 10.1016/j.burns.2017.01.017.

11. CM Stekelenburg, JM Simons, W Tuijnebreijer, PPM van Zuijlen

"Analyzing contraction of full thickness skin grafts in time: Choosing the donor site does matter"

Burns. 2016 Nov;42(7):1471-1476. doi: 10.1016/j.burns.2016.02.001. 
12. LW Waaijer, JM Simons, IHM Borel Rinkes, PJ v Diest, HM Verkooijen, AJ Witkamp

"Systematic review and meta-analysis of the diagnostic accuracy of ductoscopy in patients with pathologic nipple discharge"

Br J Surg. 2016 Mar 23. doi: 10.1002/bjs.10125.

13. JM Simons, M Teshome, KK Hunt

"Inflammatory Breast Cancer"

Book chapter accepted for Complex General Surgical Oncology in partnership with SSO.

14. LW Waaijer, MD Filipe, JM Simons, CC vd Pol, T d Boorder, PJ v Diest, AJ Witkamp "Detection of breast cancer precursor lesions by autofluorescence ductoscopy" Submitted. 
Appendices 


\section{ACKNOWLEDGEMENTS | DANKWOORD}

De afgelopen jaren heb ik met veel plezier aan dit proefschrift gewerkt en heb ik het geluk gehad onderdeel te zijn geweest van het mooie RISAS team. Gezien iedereen verspreid door het land woont en werkt, zijn er heel wat app- en telecallsessies geweest, die altijd weer energie gaven of nieuwe ideeën voortbrachten, maar zo nu en dan ook voor de slappe lach zorgden. Zo kwam ik laatst een aantekening van één van die sessies tegen waarin het ging over de roze olifant in de kamer van Linetta, de koe op het dak van Ernest en de vuilniswagen van Carmen. Ik heb geen idee meer waar het daadwerkelijk over ging, maar het herinnert me eraan hoe fijn de sfeer is binnen onze groep. Dit proefschrift is niet alleen tot stand gekomen dankzij het RISAS team, maar met de hulp van velen, zowel indirect als direct, wie ik allen heel hartelijk wil danken. Een aantal wil ik in het bijzonder bedanken.

Allereerst de patiënten die door heel het land aan de RISAS studie deelnemen. Ik vind het ontzettend bewonderenswaardig hoe zij, in een zeer moeilijk en bewogen moment in hun leven, ervoor kiezen om zich in te zetten voor het verbeteren van de zorg, zelfs wanneer ze daar zelf geen voordeel aan hebben. Dankzij en met hen kunnen we trials laten slagen.

Prof. dr. Smidt, beste Marjolein, ik kan me onze allereerste ontmoeting nog herinneren als de dag van gister, met een biertje bij de RAl met jou en Linetta. En al voelde het meteen goed, ik had toen echt niet zien aankomen dat ik in zo'n hechte en bijzondere groep terecht zou komen. Dank voor al je steun, de kansen die je me hebt geboden en gegund, je onuitputtelijke enthousiasme en je wijze raad tijdens hoogteén dieptepunten. Ik heb me geen fijnere promotor kunnen wensen. Ik heb enorm veel van je geleerd en verheug me op al het samenwerken dat nog gaat komen!

Dr. Luiten, beste Ernest, van samen op OK in Breda tot telefoonsessies in de auto, ik kan me geen moment herinneren dat er geen rake one-liner van jou voorbij is gekomen. Los van alles wat je betekent hebt voor het onderzoek en de manuscripten in dit proefschrift, kon ik ook daarbuiten voor adviezen bij je terecht. Of het nu ging om mijn Fiat of om plannen voor de toekomst, ik heb altijd van onze gesprekken genoten en er ontzettend veel aan gehad, bedankt hiervoor!

Dr. Koppert, beste Linetta, ik ben ontzettend dankbaar voor het mooie promotietraject dat ik dankzij jullie heb kunnen doorlopen. Ik bewonder je altijd aanwezige enthousiaste en optimistische instelling en met hoeveel toewijding je voor je patiënten zorgt. Bedankt voor al je begeleiding bij het onderzoek en de manuscripten en voor je aandacht voor het behouden van "mijn eigen stijl" tijdens het schrijven van de introductie en discussie. Verheug me op het opstarten van MINIMAX! 
Drs. van der Pol, beste Carmen, zonder jou was ik nooit bij RISAS terecht gekomen, bedankt dat je mij destijds bij het team hebt geïntroduceerd en betrokken. Ik ben heel trots op het mooie MARI+SWK cohort dat we uiteindelijk hebben weten te verzamelen! Ook bij jou kon ik met alles terecht, en altijd had je wel weer inspirerende adviezen (Al Pacino!). Bedankt voor al je support en je vertrouwen in mij.

Beste Thiemo, ontzettend bedankt voor hoe jij me in de wereld van het onderzoek (en vooral ook van de oksel) hebt ingewijd. Wat gaaf dat het eind van RISAS nu echt in zicht is. Bedankt voor de fijne samenwerking, ik kijk uit naar verdere projecten samen!

Leden van de beoordelingscommissie, prof. dr. Stassen, prof. dr. Pijnappel, prof. dr. Tjan-Heijnen en dr. van den Bongard, hartelijk bedankt voor jullie tijd, interesse en inspanningen voor het beoordelen van mijn proefschrift. Dank aan alle leden van de oppositiecommissie voor jullie interesse en de bereidheid hierin zitting te nemen.

Er werken ontzettend veel mensen mee aan de RISAS studie, en het lukt nooit om iedereen persoonlijk te noemen, maar evident is dat we zonder jullie nooit zo ver zouden zijn gekomen, onwijs veel dank voor al jullie inzet, hulp en betrokkenheid op welke manier dan ook. In het bijzonder veel dank aan alle lokale hoofdonderzoekers: dr. Heijmans, dr. Mares, dr. van Haaren, dr. Vles, dr. Boskamp, dr. Smit, dr. Menke, dr. Kelder, dr. Sars en dr. Contant. Ook veel dank aan verdere lokale teamleden voor alle hulp bij RISAS, onder andere Dunja van der Meer, Judith Woolschot, Janneke Hover, José Schellekens, Judith Nijhuis, Monique Kuipers, Annet Eerens, Sara van Bekkum, Tessa de Vries, Hanneke Kreiter, Jennifer Bakker, Esther Schmidt en Petra Frankhuizen. Ook aan alle pathologen, radiologen en nucleair geneeskundigen van de verschillende deelnemende centra, bedankt voor jullie betrokkenheid bij de studie!

Prof. dr. Verhoef, chirurgen van de afdeling oncologische chirurgie en alle leden van het mamma MDO, veel dank voor jullie interesse in en samenwerking binnen de RISAS studie en de altijd weer warme opvang in het Erasmus MC!

Alle secretariaten die ik veelvuldig om hulp heb gevraagd: dank aan Mariëlle, Romy en Fatiha van het UMCU, Sandra en Ada van het Erasmus MC en Sabeth van het MUMC+.

Beste co-auteurs, dank voor al jullie waardevolle input voor de verschillende manuscripten. Ik heb van onze samenwerking genoten!

Sabine de Wild, heel veel succes met het voortzetten van deze onderzoekslijn middels de MINIMAX studie, ik kijk uit naar onze samenwerking! 
Veel dank aan de sponsoren van de studies (KWF-Alpe D'HuZes, KWF-Pink Ribbon) en de organisaties die mij een reisbeurs hebben geboden (Prins Bernhard Cultuurfonds, dr. Catharine van Tussenbroek Fonds, Jo Kolk Studiefonds en EUR Trustfonds).

Dear prof. dr. Hunt, thank you for giving me the opportunity to work as a research assistant at the department of breast surgical oncology at MD Anderson Eancer Center. It has been an experience of a lifetime! Thank you for the warm welcome, for all the interesting discussions on axillary management and for involving me in all the activities in and outside of the hospital. Dear dr. Caudle, thank you for letting me participate in the exciting project on TAD with magnetic seeds! Dear Min Yi, thank you for meeting with me so often for the different projects, I really appreciate to have had the possibility to work with you. I also would like to thank all the other surgeons, fellows (Puneet, Susie, Matt and Ben) and assistants of the department, thank you for the wonderful experience I've had at MD Anderson with you.

Beste dr. Witkamp, Arjen, dank voor je vertrouwen destijds in mij om het ductoscopie-project voort te zetten. Toen bleek dat de financiering nog lang op zich zou laten wachten, was je de eerste om te zeggen dat ik voor het RISAS project moest gaan en steunde je me hierin, bedankt hiervoor. Ook prof. dr. van Diest, beste Paul, dank voor het vertrouwen destijds en nu voor de samenwerking binnen RISAS.

Dear Deta, Maira and Thalita, thank you for introducing me in the world of research. It was an unforgettable experience and I'm grateful to have met such fantastic people.

Prof. dr. van Zuijlen, beste Paul, bedankt voor je vertrouwen destijds en om mij te betrekken bij de LDI en FTG-3D studie. Ik heb ontzettend veel geleerd tijdens mijn wetenschappelijke stage in het Brandwondencentrum! Het enthousiasme van jou, Carlijn en de rest van het team, heeft mij gestimuleerd om zelf als arts-onderzoeker aan de slag te gaan. Bedankt hiervoor!

Onderzoekers van het UMCU, dank voor alle gezelligheid en het delen in alle PhD successen én frustraties. In het bijzonder de onderzoekers van de $7^{\mathrm{e}}$, Joep, Leenstar, Aanrecht, Baggio, Armallet en Marjo. Ik heb werkelijk waar een ontzettend mooie tijd met jullie gehad, waarbij onze kamers soms meer als ontmoetingsplaats/koffietent/ café/bioscoopkamer fungeerden, dan iets anders. Toch werd er ook keihard gewerkt en hebben we intussen allemaal ons proefschrift (bijna) af! Ook Leo, Walma, Connie, en DJ, bedankt voor alle gezelligheid de afgelopen jaren. Hopelijk gaan er, nu we omstebeurt de toren aan het verlaten zijn, wel nog reünies volgen! Sieske en Merel, door de afstand tussen $Q$ en WKZ zagen we elkaar niet dagelijks, maar ik heb altijd ontzettend genoten van de lunches/koffies/etentjes/etc. met jullie, veel succes met het afronden van jullie proefschrift! 
Onderzoekers van de Daniël, inmiddels NA-21, al was ik er vaak maar 1x in de week, ik heb me van begin af aan welkom gevoeld bij jullie, bedankt daarvoor! Ik heb genoten van de pecanbroodjes, lunches en natuurlijk het diner op het eiland, en sorry mannen, maar de girls-avondjes mogen hier ook niet ontbreken. En Melissa, onwijs veel dank voor al je hulp als monitor van de RISAS studie! Succes met al jullie mooie projecten en hopelijk zie ik jullie gauw weer eens!

Onderzoekers uit Maastricht, van congressen in Seattle en Barcelona tot Coldplay in Brussel en de bbq's bij Marjolein thuis, het is met jullie altijd een feestje. Dank voor al jullie gezelligheid en natuurlijk ook voor de hulp met RISAS. Succes met alles, tot snel!

Alle arts-assistenten, PA-ers, fellows en stafleden van Meander Medisch Centrum, UMC Utrecht en St. Antonius Ziekenhuis, bedankt voor de fijne en leerzame tijd die ik bij jullie heb gehad in de kliniek.

Dear John, I couldn't have asked for a better roommate during my stay the first month in Houston. It was great exploring the city together with you and Tanvir. And of course, sharing our love for eating (a lot), I'll never forget the BBQs on the rooftop!! Tanvir, you have such an infectious enthousiasm for Houston. Thank you for showing me around and letting me stay at your place. I'm so glad to have met you guys and hope to see you again soon (somewhere in the world)!!

Lieve meiden van de Springplank, wat bijzonder dat we nog steeds vriendinnen zijn. We kennen elkaar inmiddels al bijna 30 jaar en alhoewel iedereen een andere weg is ingeslagen, zijn de momenten met jullie samen nog altijd even vertrouwd als vroeger! Hoop dat we dit voor altijd vasthouden!

Lieve Ania, ons contact is niet altijd even intensief geweest de afgelopen jaren, maar we zijn elkaar gelukkig nooit uit het oog verloren. Bedankt voor je fijne en bijzondere vriendschap en ik kijk uit naar de dag dat jij mag promoveren!

Lieve Tapasclub, van weekendjesweg tot themafeestjes en van zeer professionele wijnavonden tot onze eigen boekenreeks!! Kijk nu al uit naar de nog komende events:D Kirs, ook al behoort het Surinameplein nu echt tot het verleden, ik zal de fantastische tijd en avonturen die wij hebben beleefd nooit vergeten!!

Mijn paranimfen, Daniëlle en Lindsay, wat ben ik blij dat jullie deze dag naast mij staan. Daniëlle, we zijn al zolang ik me kan herinneren vriendinnen en het is heel fijn te weten dat dit voor altijd is! Onze eerste echte vakantie zonder ouders in TerSpegelt, samen naar het Grand Café, studeren in Amsterdam, Kos, varkensbiggen en sneeuwpop Henk, de herinneringen zijn eindeloos. Ik vind het heel bijzonder om jou nu ook als moeder te zien, ik ben super trots op je en zoals jij laatst ook weer eens zei: hopelijk zitten we later samen in het bejaardentehuis:) Lindsay, al vanaf de eerste 
dag op de VU was onze vriendschap een feit en gelukkig nog steeds! Ik bewonder hoe gedisciplineerd en vastberaden jij te werk gaat en hoeveel aandacht je altijd hebt voor de mensen om je heen. Ik geniet van alle avondjes samen met lekker eten en wijntjes en verheug me nu al op alle die nog komen gaan! Nu op naar jouw promotie!!

Queridos María y Miguel, gracias por acogerme tan cálidamente desde el principio. Siempre es un placer estar con vosotros en España y, por supuesto, recorrer los Países Bajos juntos. Ahora que mi tesis doctoral se ha completado, espero tener más tiempo para finalmente aprender bien el español. Jorge and Miguel, it is always nice seeing you, whether it is in Coruna, Barcelona or Amsterdam! Let's plan the next trip soon!

Lieve Reem, brotha, m'n broertje, of beter gezegd, chef Simons. Wij hebben natuurlijk beide geen betere zus of broer kunnen wensen. Gelukkig is de tijd van elkaar de hersens in slaan (zo goed als) voorbij. Ik ben ontzettend trots op wat je allemaal al bereikt hebt en bewonder hoe hard je hiervoor werkt. Ik kijk uit naar alles wat we samen nog gaan meemaken, alhoewel het voor iedereen waarschijnlijk beter is als we een vakantie met alleen ons tweetjes nooit meer herhalen. Love you for ever! Lieve Sannah, wat ontzettend leuk om jou bij onze familie te hebben. Altijd gezellig, attent en vol energie. Jij en $\mathrm{m}^{\prime} \mathrm{n}$ broertje zijn een top stel en de tripjes met ons viertjes zijn altijd weer fantastisch en geslaagd! Hopelijk volgen er nog velen!

Lieve mam en pap, wat hebben wij toch een mooi, bijzonder en liefdevol gezin met elkaar. Jullie hebben werkelijk waar altijd voor Remi en mij klaar gestaan en nog steeds kunnen we voor alles bij jullie terecht (gelukkig niet alleen voor goede raad, maar ook nog steeds voor huisgemaakte pizza en kip met boursin). Bedankt dat jullie er altijd voor ons zijn, voor alle levenslessen, normen en waarden en voor jullie onvoorwaardelijke steun, ik heb me geen betere ouders kunnen wensen en ik houd ontzettend veel van jullie!

Lieve Pablo, wat ben ik blij dat jij destijds besloten hebt om naar Nederland te verhuizen (en dat ik besloot opnieuw een beugel te nemen). Het had zo moeten zijn! Samen met jou voel ik me ontzettend gelukkig, zoals Mario Benedetti ware liefde al eens zo mooi beschreef:

Si te quiero es porque sos, mi amor mi complice y todo y en la calle codo a codo, somos mucho más que dos

Bedankt dat je er altijd voor me bent Pablo, ik houd heel veel van je en ik kijk enorm uit naar de toekomst samen met jou! 
Appendices 


\section{CURRICULUM VITAE}

Janine Simons was born on the 18th of August, 1989, in Naarden and grew up in Huizen. In 2007 she finished high school (Erfgooiers College) and started medical school at the Vrije Universiteit in Amsterdam. In the second year, she was selected to participate in a summer research exchange project at the Centro de Onco-Hematologia Pediátrica (University of Pernambuco) in Recife, Brazil. During her master studies she did an extra-curricular internship in Tanzania, at Machame Hospital in the Kilimanjaro region. Her interest in research was stimulated by her research internship at the Burn Center Beverwijk (Rode Kruis Ziekenhuis). After she

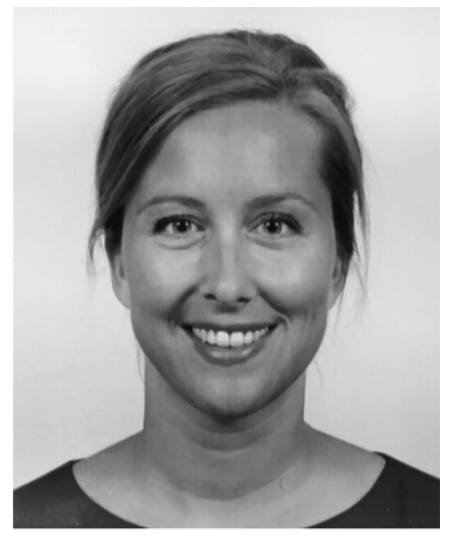
obtained her medical degree in 2014, she started working as a surgical resident (not in training) at the Meander Medical Center in Amersfoort. She also worked at the Intensive Care department for three months. Subsequently, she continued working as a surgical resident at the University Medical Center in Utrecht. During this residency, she did research on ductoscopy and wrote several grant proposals. Following her ambitions to pursue a $\mathrm{PhD}$, she applied for a full-time clinical research position on axillary management in breast cancer, supported by the Dutch Cancer Society. She started as a PhD Candidate in May 2016, under the supervision of Marjolein Smidt, Ernest Luiten, Linetta Koppert and Carmen van der Pol. As part of this position, she set up a prospective trial and initiated this trial at 14 Dutch institutions. She furthermore initiated multiple collaborations with other specialists and institutions both national and international. In 2018, she received several travel grants, which made it possible to work as a research assistant for five months at the department of breast surgical oncology at MD Anderson Cancer Center in Houston, under the supervision of dr. Hunt. Janine is looking forward to, after obtaining her PhD, combining a clinical position with postdoctoral research. In her spare time, she loves to go traveling and to spend time with friends and family. Janine is living together with Pablo in Amsterdam. 
